1983

\title{
Long-time states of inverse cascades in the presence of a maximum length scale
}

\author{
Murshed Hossain \\ College of William \& Mary - Arts \& Sciences
}

Follow this and additional works at: https://scholarworks.wm.edu/etd

Part of the Plasma and Beam Physics Commons

\section{Recommended Citation}

Hossain, Murshed, "Long-time states of inverse cascades in the presence of a maximum length scale" (1983). Dissertations, Theses, and Masters Projects. Paper 1539623742.

https://dx.doi.org/doi:10.21220/s2-j1gp-ex34

This Dissertation is brought to you for free and open access by the Theses, Dissertations, \& Master Projects at W\&M ScholarWorks. It has been accepted for inclusion in Dissertations, Theses, and Masters Projects by an authorized administrator of W\&M ScholarWorks. For more information, please contact scholarworks@wm.edu. 


\section{INFORMATION TO USERS}

This reproduction was made from a copy of a document sent to us for microfilming. While the most advanced technology has been used to photograph and reproduce this document, the quality of the reproduction is heavily dependent upon the quality of the material submitted.

The following explanation of techniques is provided to help clarify markings or notations which may appear on this reproduction.

1. The sign or "target" for pages apparently lacking from the document photographed is "Missing Page(s)". If it was possible to obtain the missing page(s) or section, they are spliced into the film along with adjacent pages. This may have necessitated cutting through an image and duplicating adjacent pages to assure complete continuity.

2. When an image on the film is obliterated with a round black mark, it is an indication of either blurred copy because of movement during exposure, duplicate copy, or copyrighted materials that should not have been filmed. For blurred pages, a good image of the page can be found in the adjacent frame. If copyrighted materials were deleted, a target note will appear listing the pages in the adjacent frame.

3. When a map, drawing or chart, etc., is part of the material being photographed, a definite method of "sectioning" the material has been followed. It is customary to begin filming at the upper left hand corner of a large sheet and to continue from left to right in equal sections with small overlaps. If necessary, sectioning is continued again-beginning below the first row and continuing on until complete.

4. For illustrations that cannot be satisfactorily reproduced by xerographic means, photographic prints can be purchased at additional cost and inserted into your xerographic copy. These prints are available upon request from the Dissertations Customer Services Department.

5. Some pages in any document may have indistinct print. In all cases the best available copy has been filmed.

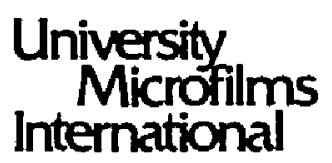

300 N. Zeeb Road

Ann Arbor, MI 48106 

8407035

Hossain, Murshed

LONG-TIME STATES OF INVERSE CASCADES IN THE PRESENCE OF A MAXIMUM LENGTH SCALE

The College of William and Mary in Virginia

PH.D. 1983

\section{University Microfilms \\ International 300 N.Zeeb Road, Ann Arbor, M148106}


. 
PLEASE NOTE:

In all cases this material has been filmed in the best possible way from the available copy.

Problems encountered with this document have been identified here with a check mark

1. Glossy photographs or pages

2. Colored illustrations, paper or print

3. Photographs with dark background

4. Illustrations are poor copy

5. Pages with black marks, not original copy

6. Print shows through as there is text on both sides of page

7. Indistinct, broken or small print on several pages

8. Print exceeds margin requirements

9. Tightly bound copy with print lost in spine

10. Computer printout pages with indistinct print

11. Page(s)____ lacking when material received, and not available from school or author.

12. Page(s) seem to be missing in numbering only as text follows.

13. Two pages numbered . Text follows.

14. Curling and wrinkled pages

15. Other

\section{University Microfilms International}




1
1
1
1
1
1


LONG-TIME STATES OF INVERSE CASCADES IN THE PRESENCE OF

A MAXIMUM LENGTI SCALE

\begin{abstract}
A Dissertation
presented to

The Faculty of the Department of Physics

The College of William and Mary in Virginia
\end{abstract}

In partial fulfillment

of the requirement for the Degree of

Doctor of Philosophy

by

Hurshed llossain

November 1983 
APPROVAL SHEET

This dissertation is submitted in partial fulfillment of the requirement for the degree of Doctor of Philosophy

Murahed Amain

Nurshed Hossain

Approved, November 1983
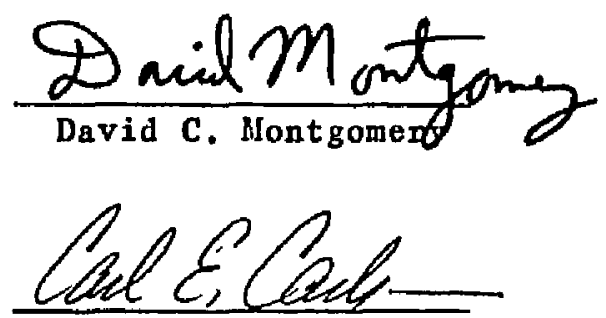

Carl E. Carlson

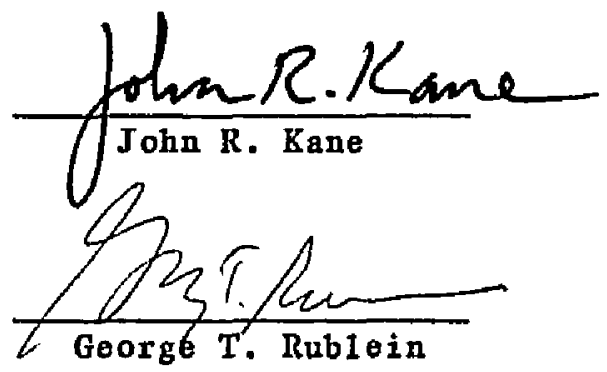

s. Valet

George M. VahaIa

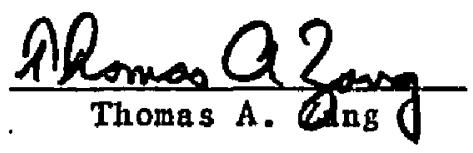


TABLE OF CONTENTS

ACKNOWLEDGEMENT

iv

ABSTRACT

$\ldots \ldots \ldots \ldots \ldots \ldots \ldots \ldots, v$

I. INTRODUCTION

1

II. STATEMENT OF THE PROBLEM AND METHOD OF SOLUTION $\ldots 8$

III. CASCADES AND INVERSE CASCADES

$\ldots \ldots \ldots \ldots 23$

IV. RESULTS

a) EQUILIBRIUM RUNS $\quad \ldots \ldots \ldots \ldots \ldots 30$

b) DISSIPATIVE FORCED NAVIER-STOKES RUN $\ldots \ldots \ldots \ldots \ldots 31$

c) DISSIPATIVE FORCED IHD RUNS $\ldots \ldots \ldots \ldots \ldots 35$

d) PIIASE RANDOMIZED RUN (NAVIER-STOKES) $\ldots \ldots \ldots \ldots \ldots 39$

v. ALGEBRAIC MODEL FOR THE FINAL STATES $\ldots \ldots \ldots \ldots \ldots 41$

APPENDIX A.

NON-DISSIPATIVE PREDICTIONS $\ldots \ldots \ldots \ldots \ldots \ldots \ldots .46$

REFERENCES $\quad \ldots \ldots \ldots \ldots \ldots \ldots \ldots \ldots \ldots \ldots \ldots \ldots, 53$

FIGURES $\quad \ldots \ldots \ldots \ldots \ldots \ldots \ldots \ldots \ldots \ldots \ldots \ldots, 58$

iii 


\section{ACKNOHLEDGEBENTS}

I would like to express deep appreciation to Professor David C. Montgomery for suggesting this problem and for his continued guidance during the years of my research at the College of William and Mary. I would like to thank the late Professor Frederic Crownfield, Dr. George Vahala, Dr. Thomas Zang and Dr. William Matthaeus for many encouraging and enlightening discussions.

I would like to thank Donald Joyce, Muhamed Numan, David Reed, Samuel Hoyle and Betru Debebe for their help in computer related matters. I would also like to thank Jill Dahlburg, Russe11 Dahlburg and Hudong Chen, the fellowmembers of our research group, for many detailed discussions on various problems and hours of co-operation.

Thanks are also due to Vina Punjabi, Alkesh Panjabi, Muhamed Numan, Saiful Huq, Betru Debebe and Vanamali Raghunathan for their friendship that has been very important during my years of study at the College.

Finally I express my appreciation to my wife Sufia Khatun whose encouragement and understanding made this work end we11. 
It is shown numerically, both for the two-dimensional NavierStokes equations and for two-dimensional magnetohydrodynanics, that the long-time asymptotic state in a forced inversecascade situation is one in which the spectrum is completely dominated by its own fundamental. The growth continues until the fundamental is dissipatively limited by its own dissipation rate. An algebraic model is proposed for the dynamics of such a final state. 
LONG-TIME STATES OF INVERSE CASCADES IN THE PRESENCE OF A MAXIMUM LENGTH SCALE 


\section{Chapter I}

\section{INTRODUCTION}

It is a we11-known fact that under suitable conditions some motions of a magnetofluid are such that the velocity and magnetic fields at any time and position are not found to be the same when they are measured several times under seemingly identical conditions. In these motions variables take random values which are not determined by the macroscopic data. These motions are called turbulent motions.

Magnetohydrodynamic turbulence theory is a recent subject whtle fluid turbulence has a long history, almost half a century.

The beginning of modern turbulence theory may be attributed to Taylor (1935) when the continuous nature of random velocities was recognized (Batchelor 1953). Taylor introduced the concept of correlation between the velocities at two points as one of the most important quantities needed to describe turbulence. The Fourier transform of one such correlation is the spectrum function (Batchelor 1953, Monin and Yaglom 1971, Panchev 1971). The next step was to simplify the turbulence with the assumptions of homogeneity and isotropy. The first assumption neans that the statistical properties of the velocity field and all its moments are independent of position. Isotropy assumes the statistical properties to be independent of any preferred direction. Both of these assumptions were proven to be approximately true for turbulence generat- 
ed by a regular array of rods in a wind tunnel (Corrsin 1962, Hinze 1975).

Taylor demonstrated in 1938 the action of the non-linear terms (Batchelor 1953), namely, to mix components of turbulence having different length scales.

In 1937 Karmán and Howarth (1938) showed that mean values of the product of two of the three components of velocities at two different points can be expressed in terms of a single scalar function when the turbulence is isotropic. Robertson showed in 1940 that an isotropic tensor of any order conld be expressed in terms of the known invariants of the rotation group. No progress had been made in understanding the underlying physical process until 1941 when Kolmogoroff suggested a similarity hypothesis. He hypothesised that small scale components of turm bulence are approximately in statistical equilibrium and that the equilibrium is aniversal, apart from the effect of variation of two parameters, one the viscosity and the other determined by the large scale components of the turbulence (namely the rate of energy supplied by the large scalo components). Given these two parameters he predicted properties of small scale components of the turbulence from dimensional analysis, e.g. the distribution of energy over the wave numbers within the equilibrium range varies as $k^{-5 / 3}$.

Burgers took a simplified problem - the one-dimensional counterpart of the Navier-Stokes equation which is named after him - and revealed the effects of the non-1inear and viscous terms in producing and reducing velocity gradients respectively. 
In parallel to this theoretical progess many measurements have been made to prove or disprove theories or to reveal Hew physics. Among many works Townsend (e.g. 1947) may be mentioned for the kinds of measurements that were possible with the help of hot-wire anemometer.

Simultaneously purely mathematical problems associated with random functions were also addressed and turbulence theory absorbed them. We can refer to Robertson(1940) for the kinematics of correlation tensor for isotropic turbulence and to Batche1or(1946) and Chandrasekhar(1950) for axisymmetrio turbulence.

Including a number of incomplete lines of research none of the above could explain turbulent behavior of fluid to a satisfactory extent. In the 1960 s computer application in science spread very fast. Numerical solutions of complicated mathematical problems became possible. New trends in extracting new physics and explaining known phenomena began. The study of fluid and plasma turbulence in the last two decades has been mostly numerical. Present day computational facilities are still not adequate to start an extensive study of three-dimensional turbulence. Two-dimensional simulation is now very common and justification for such study has been given many times (Batchelor 1969, Kraichnan and Hontgomery 1980). Besides its applicability in geophysical phenomena in the atmosphere, ocean and magnetosphere, two-dimensional turbulence has in common the two basic characteristics of three-dimensional turbulence, namely, randomness and non-1inearity. A review of two-dimensional turbulence may be found in Kraichnan and Hontgomery (1980). 
Many turbulence studies including this one assume incompressibility. Hence interesting phenomena such as shock and sound wave propagation are excluded. Nevertheless systems having flow speeds much less than that of sound happen to satisfy tho incompressibility criteria pretty we11. Yet there are some cases where the incompressibility is satisfied while the above condition is not. Montgomery (1982) showed a different approach to understand this situation. His argument is based on an estimate of transport coefficients. The form of the dissipative terms are $\nabla .\left(\eta_{0} \nabla \vee\right)$. The transport coeffient $\eta_{0}$ is estimated to be very large. So in order for the system not to be completely overwhelmed by dissipative actions only, $\nabla . y$ has to be very small.

The two-dimensional Navier-Stokes system has been investigated anaIytical1y by Onsager(1949),Fjortoft (1953), Kraichnan(1967), and Leith (1968) among others. Starting from a truncated system of two-dimensional equations it can be shown (details are stated in Appendix A) that there exists a Liouville theorem if dissipation is set equal to zero. It then follows that there exists an equilibrium statistical mechanics predicting thermal equilibrium of the system. Similar results were later predicted for magnetohydrodynamics by Fyfe et a1 (1976). Both of these predictions were varified numerically (Seyler et a1 1975, Fyfe et al $1977 \mathrm{a})$. Details are presented in Appendix $A$. We have reproduced these results as a test of our codes. Results are presented in Chapter IV.

No real physical system however is non-dissipative. In the presence of dissipation the system decays in time along with a reshuffing of on- 
ergy among various wavenumber components. In these decay processes or in a driven system, cascades of global quantities are proposed. Details are described in Chapter III. In the three-dimensional Navier-Stokes system energy cascades to short wave lengths, while in two-dimensions a dual cascade is proposed. These results are numerically verified qualitatively by Fyfe et al(1977b). Under these circumstances energy cascades to long wave lengths along with an enstrophy cascade to short wave lengths (enstrophy being the integrated squre vorticity).

Numerical studies so far done mostly had emphasised the power-law behavior for wave-number spectra, following the influential conjecture of Kraichnan(1967) concerning two possible inertial subranges. Some investigations of dissipation scale behavior have also been reported for two-dimensional MHD case Corszag and Tang 1979, Matthaeus and Montgomery 1980, 1981, Matthaeus 1982, Frisch et a1 1983). Power laws are obtained persuasively only from dimensional analysis. In order for them to be seen clearly, they are thought to require very large Reynolds numbers (mechanical and/or magnetic) in order that the inertial subranges be clearly separated from each other and from the dissipation range. High spatial resolution is required at these large Reynolds numbers, and it is accurate to say that no reported numerical solution has contained a wide enough range of wave numbers to satisfy the chain of inequalities required for a satisfactory test of the power law behavior in any putatively inverse-cascading situation. There is no question, however, that the qualitative effect of large back-transfer (transfer to 1ong wave1engths) has beon seen in numerical solutions of the relevant dynamical equations (Li1ly 1969, Fyfe et al 1977b, Meneguzzi et a1 1981). 
Inverse cascades are of more than academic interest. Both in the atmosphere and in thermonuclear confinement machines there is no end to sources of small scale disturbances similar to the effect of driving the system in a band of wavenumbers in numerical simulation. These sma11 scale excitations may lead to large scale effects through inverse cascades.

Theoretical predictions typically have been for unbounded systems, so that there were arbitrarily many additional octaves in wavelengths for the spectrum of the injected ard inversely-cascaded quantity to expand into at the lower end. Numerical solutions, however, always take place on periodic or finite grids, and thereby have associated with them a maximum wave length (minimum wave number) beyond which the inverselycascaded spectrum cannot go. Because of the slow time scales involved in the long wave length eddies and because of limited computer budgets, computations have not been reported for times long compared to the time required for the longest allowed wave length to fill up. Both theory and computation have remained vague on the question of what happens in an inversely-cascading situation.

In this study, we have addressed this question numerically for both two-dimensional Navier-Stokes and MHD cases. In both cases, the fields are driven by a prescribed random function, band-1imited in wavenumber space, which serves as the sources of the excitations that are transferred elsewhere in the $\underset{\sim}{k}$ space. Details are presented in Chapter II. The resolution of the computation is not high and the Reynolds numbers are not large. There is no pretense that the inertial subranges are well 
separated or that the power law predictions are verifiable. It is not necessary, however, to be in this high Reynolds number regime in order to see large amounts of back transfer, or to address the question of what happens to the back-transferred excitations when they have no lower place in wave number space to which to migrate. It is toward this 1atter qualitative question that the following study is directed.

There would seem to be at least three possible scenarios for what might occur after the $\mathrm{k}$ spectrum fills in between the forcing band and the fundamental lowest wavenumber $k_{\text {min }}$. (1) The inverse spectral transfer might cease (2) The relevant part of the $\underset{\sim}{k}$ spectrum might all rise together, possibly maintaining a power law behavior, with perhaps a 'healing region' just below the forcing band (3) The fundamental $\mathrm{k}_{\text {min }}$ might continue to absorb the supplied excitations, running of $f$ and leaving the rest of the spectrum, until it is limited by its own dissipation rate. These three may not exhaust the possibilities, but what we wish to demonstrate in this study is that alternative (3) is in fact what occurs both for two-dimensional Navier-Stokes (2D NS) and magnetohydrodynamic (2D MHD) cases. 


\section{Chapter II}

\section{STATEMENT OF THE PROBLEM AND BETHOD OF SOLUTION} ing.

The equations of incompressible magnetohydrodynamics are the follow-

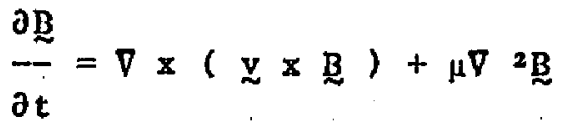

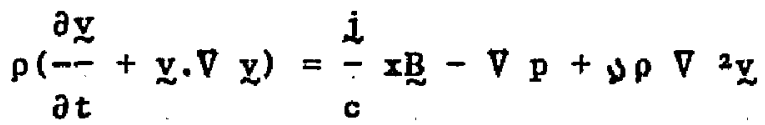

$$
\begin{aligned}
& \nabla \times B=\frac{4 \pi}{c} i \\
& \nabla \cdot \underline{B}=0 \\
& \nabla \cdot \mathbf{y}=0
\end{aligned}
$$

Omission of equations $(2.1 a),(2.1 c),(2.1 d)$ and setting $B=0$ leave us with the incompressible Navier-Stokes equations. The magnetic and velocity fields are $\mathbb{R}$ and $y$ respectively. The current density is $i$, the pressure is $p$ and the density is $\rho$. The magnetic diffusivity is $\mu=c^{2} / 4 \pi \sigma$ ( $\sigma$ being the conductivity) and the kinematic viscosity is $\gg$. The speed of light is $c$. 
We introduce dimensionless variables in the following way. Velocities are measured in units of typical speed $U_{0}$ which may be the r.m.s. speed of the turbulent velocity field. Lengths are measured in units of $L_{0}$ which may be a characteristic macroscopic length of the turbulent field. Times axe measured in units of $\mathrm{L}_{0} / \mathrm{U}_{0}$ and the magnetic field in units of $B_{0}=\left(4 \pi \rho U_{0}^{2}\right)^{1 / 2}$. The current densities are measured in units of $\mathrm{j}_{0}=\mathrm{cB}_{0} / 4 \pi \mathrm{L}_{0}$ That is

$$
\begin{aligned}
& U=U_{0} \tilde{U} \\
& L=L_{0} \mathbb{L} \\
& t=L_{0} / U_{0} \tilde{t} \\
& B=B_{0} B \\
& j=j_{0} J \\
& p=p_{0} \tilde{P}
\end{aligned}
$$

The variables with 'tildes' are dimensionless. Then equations (2.1a-21.e) become the following.

$$
\begin{aligned}
& \frac{\partial B}{\partial t}=\nabla \times(\underset{\sim}{\mathbb{Z}} \underset{\sim}{\mathrm{B}})+\frac{1}{\mathrm{R}_{\mathrm{m}}}{ }^{2} \mathrm{~B} \\
& \frac{\partial \underline{y}}{\partial t}=-\underline{X} \cdot \nabla \underline{y}+\underset{i}{\operatorname{B}}-c_{s}{ }^{2} \nabla p+\frac{1}{R_{e}} \nabla^{2} \underline{V} \\
& \nabla \times \underline{B}=\mathfrak{i} \\
& \nabla \cdot \underline{\mathbf{B}}=\mathbf{0} \\
& \nabla \cdot \mathrm{z}=0
\end{aligned}
$$


Here all variables are dimensionless, and the tildas have been dropped. Rm and Re are the magnetic and mechanical Reynolds numbers respectively.

$$
\begin{aligned}
& \mathrm{Rm}=\frac{\mathrm{L}_{0} \mathrm{~J}_{0}}{\mu} \\
& \mathrm{Re}=\frac{\mathrm{L}_{0} \mathrm{U}_{0}}{\nu}
\end{aligned}
$$

$c_{S}$ is the speed of sound in dimensionless units and is $\left(p_{0} / \rho U_{0}^{2}\right)^{1 / 2}$

Before we express the dynamical equations in computationaliy convenient form we introduce the geometry that we assumed. This is a two-dimensional case with the following field configuration.

$$
\begin{aligned}
& B=\left(B_{x}, B_{y}, 0\right) \\
& \dot{y}=\left(v_{x}, v_{y}, 0\right) \\
& \dot{L}=\nabla \times \underset{B}{B}=\left(0,0, j_{z}\right) \\
& \mathcal{L}=\nabla \times x=\left(0,0, \omega_{z}\right) \\
& a=\left(0,0, a_{z}\right)
\end{aligned}
$$

where the vector potential a is defined by

$$
\nabla \times \mathrm{a}=\underline{\mathrm{B}}
$$

All quantities are independent of the $z$ co-ordinate, so that

$$
\frac{\partial}{\partial z}=0
$$


Taking the curl of equation (2.3b), using (2.5d), (2.3e) and some vector identities we have

$$
\frac{\partial \omega_{z}}{\partial t}=-x \cdot \nabla \omega_{z}+\underset{\sim}{B} \cdot \nabla \underset{j}{ }+\frac{1}{\operatorname{Re}} \nabla^{2} \omega_{z}
$$

With some manipulations equation $(2.3 a)$ reduces to

$$
\nabla \times\left[\frac{\partial \mathrm{g}}{\partial t}=\mathrm{y} \times(\nabla \times \mathrm{g})-\frac{1}{\mathrm{Rm}} \nabla \times \nabla \times \mathrm{g}\right]
$$

If we 'uncur1' this equation we have

$$
\frac{\partial \mathrm{a}}{\partial \mathrm{t}}=\mathrm{yx}(\nabla \times \mathrm{g})-\frac{1}{\mathrm{R}_{\mathrm{m}}} \nabla \times \nabla \times \mathrm{g}+\nabla \emptyset
$$

where $\emptyset$ is an arbitrary scalar function. If we choose the Coulomb gauge then $\emptyset$ is the scalar potential satisfying the following equation in laboratory units.

$$
E=\nabla\left(\frac{\emptyset}{c}\right)-\frac{1}{c} \frac{\partial \mathrm{a}}{\partial t}
$$

In our geometry $a=\left(0,0, a_{z}\right)$ and hence the Coulomb gauge is identically satisfied. We exploit this freedom to choose $\nabla \emptyset=0$ which amounts to neglecting the electrostatic field. After doing some vector manipulations equation $(2.7)$ reduces to 


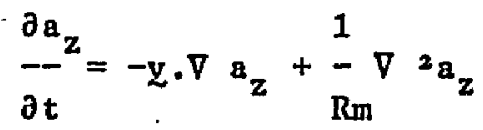

Equations (2.6) and (2.9) along with the solenoidal and incompressiblity conditions form the set we want to solve numerically. This form of the equations is computationally convenient since they are expressed in terns of two scalars only. Moreover if the incompressibility and solenoidal conditions axo satisfied at the initial time they are automatically satisfied at all times.

Due to the two dimensional geometry we have considered we can introduce a stream function $\emptyset$ such that

$$
\nabla 2 \emptyset=-\omega_{z}
$$

so that

$$
y=\nabla \emptyset \times \hat{e}_{z}
$$

Similarly we can write for the magnetic field

$$
\nabla{ }^{2} a_{z}=-j_{z}
$$

and

$$
B=\nabla a_{z} \times \hat{e}_{z}
$$

Since we are going to study cascades, we would like to inject mechanical and/or magnetic energy to the system. lie do it by introducing forcing terms $F$ and $G$ in the two dynamical equations (2.6) and (2.9). So we have 


$$
\begin{aligned}
& \frac{\partial \omega_{z}}{\partial t}=-\underset{\sim}{\partial t} \nabla \omega_{z}+\underset{\sim}{B} \cdot \nabla j+\nu \nabla w_{z}+F \\
& \frac{\partial a_{z}}{\partial t}=-X \cdot \nabla a_{z}+\mu \nabla a_{z}+G
\end{aligned}
$$

TECHNIQUE FOR THE SPACE PART OE TIE DYNAMICAL EQUATIONS:

Now we assume rectangular periodic boundary condition, if not for any physical reason, at least for simplicity and tractability of solution. All fields are periodic in $2 \pi$. Therefore we can expand them in Fourier series with wavenumbers $k=\left(k_{x}, k_{y}\right)$ with integer components.

$$
\begin{aligned}
& \omega_{z}(x, y)=\sum_{k} \omega(\underset{\sim}{k}, t) \exp (i \underset{i}{k} \cdot x) \\
& a_{z}(x, y)=\sum_{k} a(k, t) \exp (i \underset{\sim}{k}, x)
\end{aligned}
$$

There are similar transforms for $\mathrm{z}, \mathrm{B}, \emptyset$ and $\mathrm{j}_{\mathrm{z}}$.

The Fourier coefficients have to be conjugate symmetric, for example $\omega(-k)=\omega^{*}(k)$, in order to have real values of the field variables.

Ideally the sum should run from $-\infty$ to $+\infty$. But computationally we can not have an infinite set of numbers. So one has to truncate the sum. Allow $k$ such that $k_{x}$ or $k_{y}$ can lie between $-N / 2+1$ and $N / 2$. In other words the Fourier coefficients are assumed to be zero beyond some value of wave vector $\mathrm{k}$. In doing so we lose some dynamics. Consider $j u s t$ the 
-direction. Higher order interactions (i.e. coupling between two high $k_{x}$ modes) and the coupling between a high $k_{x}$ (i.e. outside the box) and a low $k_{x}$ (i.e. within the box) are neglected. The high $k_{x}$-modes that are excited due to interaction betweon two allowed $10 w-k_{x}$ modes are also suppressed. This is a limitacion wo have to accept.

The non-linear terms appear as quadratic convolution suns in wavenumber space. Efficient evalation of convolution sums is central in being able to solve a non-linear problem numerically. A direct evaluation of a convolution sum requires order $\mathrm{N}^{*}$ operations in two-dimensions. It seoms almost impossible to use spectral methods. In 1971 an efficient way to compute the non-linear terns was proposed (Orszag 1971). In this method, known as transform method (E1iasen 1970, Orszag 1971, Patterson and Orszag 1971, Gottlieb and Orszag 1977), evaluation of a quadratic non-1inear term requires order $\mathrm{N}^{2}$ ln $\mathrm{N}$ operations.

The key to the transform method is the Discrete Fourier Transform(DFT). With the kind of truncation we have described, i.e., $k_{x}$ and $k_{y}$ 1ying between $-N / 2+1$ and $N / 2$, the $x$-space is discretized.

$$
x_{j}=\frac{2 \pi}{N} j \quad j=0,1,2, \ldots \ldots \ldots \ldots(N-1)
$$

If we have to evaluate the following non-linear term

$$
\omega(\mathrm{k})=(\mathrm{uv})_{\mathrm{k}}=\sum_{\mathrm{p}+\mathrm{g}=\mathrm{k}} \mathrm{u}(\mathrm{p}) \mathrm{v}(\mathrm{g})
$$


we transform $u(p)$ and $v(g)$ to $x$-space, multiply them together, and transform back to k-space.

$$
\begin{gathered}
u(x)-\frac{F F T}{F F T} u(x) \\
v(g)-v(x) \\
\text { calculate } \omega(x)=u(x) v(x) \\
\omega(x) \quad \text { Inverse FFT } \omega\left(\frac{x}{*}\right)
\end{gathered}
$$

This method has proven to be very useful in turbulence computations. In particular this technique became very efficient after the introduction of Fast Fourier Transform (FFT).

In the process of applying this technique to finite Fourier series aliasing exror is introduced. The quantity we intend to calculate is

$$
\begin{aligned}
& \omega(k)=\sum u(p) v(g) \\
& \mathrm{p}+\mathrm{g}=\mathrm{k} \\
& \| \mathbb{L} \mid \leqslant N / 2 \\
& \| g \mid \leqslant N / 2 \\
& \||k| \leqslant N / 2
\end{aligned}
$$

$\|\mathrm{p}\| \leqslant N / 2$ denotes that both $-N / 2+1<\mathrm{p}_{\mathrm{x}}<\mathrm{N} / 2$ and $-\mathrm{N} / 2<\mathrm{p}_{\mathrm{y}}<\mathrm{N} / 2$. The quantity which is calculated by a straight forward application of the transform technique is

$$
\omega^{\prime}(k)=1 / N^{2} \sum\left[\sum v(p) \exp (i p . x)\right]\left[\sum v(g) \exp (i g \cdot x)\right] \exp (-i k \cdot x)
$$




$\begin{array}{rlrl}= & \sum u(p) v(g) & \text { if } \underset{k}{\mathrm{k}}=(\mathrm{p}+q) \bmod \underset{N}{N} \\ & \|\mathrm{~g}\| \leqslant N / 2 & \\ & \|\mathrm{~g}\| \leqslant N / 2 & \\ = & 0 & & \text { otherwise }\end{array}$

That is

$$
\omega^{\prime}(k)=\omega(k)+\omega\left(k_{x} \pm N, k_{y} \pm N\right)+\omega\left(k_{x} \pm N, k_{y}\right)+\omega\left(k_{x}, k_{y} \pm N\right)
$$

For any fixed ||$k \mid l \leqslant N / 2$ not all combination of + and - are allowed. For example, if $k_{x}=0$ and $k_{y}=N / 2$ then only $\omega\left(k_{x}+N, k_{y}-N\right)\left(k_{x}+N, k_{y}\right)$ and $\left(k_{x}, k_{y}-N\right)$ can be non-zero. Because $p$ and $g$ can add up to give components lying between $-\mathrm{N}+2$ and $\mathrm{N}$ only. In the expression for $\omega^{\prime}(\underset{\sim}{\mathrm{k}})$ we have aliased terms in addition to the correct value. The second term on the right hand side is a doubly aliased term where both $k_{x}$ and $k_{y}$ are aliased. The last two terms are singly aliased terms where either $k_{x}$ or $k_{y}$ is aliased.

Orszag(1971), and Patterson and Orszag(1971) suggested a way out. The most efficient method to de-alias $\omega^{\prime}(\underline{k})$ is to apply the method of isotropic truncation for the second term on right hand side and the shifted grid method for the last two terms.

For the aliasing of the second kind $i . e$. either $k_{x}$ or $k_{y}$ is aliased (singly aliased) we introduce a set of shifted grids

$$
x^{s}\left(j_{1}, j_{2}\right)=\frac{2 \pi}{N}\left(j_{1}+1 / 2, j_{2}+1 / 2\right)
$$

in addition to the standard grid 
$x\left(j_{1}, j_{2}\right)=\frac{2 \pi}{N}\left(j_{1}, j_{2}\right)$

where $j_{1}, j_{2}=0,1,2, \ldots \ldots \ldots \ldots(i-1)$.

If we transforn $u(g)$ and $v(g)$ to the shifted grid, perforn the aultiplication and cone back to $k$-space as " $s\left(\begin{array}{l}n \\ \approx\end{array}\right)$ then

$$
1 / 2\left[w^{\prime}(k)+\exp (-i \pi k \cdot \hat{e} / \cdots) w_{s}(k)\right]=w(k)+w\left(k_{x}+i, k_{y}+H\right)
$$

where $\hat{e}=(1,1)$. That is we iave corrected the singly aliased terms by doing threc additional transforms.

To retrove the crror in filicit both $k_{\pi}$ and $k_{y}$ ins been aliased we notice that the $g^{\prime} s$ and $q^{\prime} s$ chat give rise to such an error theraselves have to be on the edge of the truncated l-space.

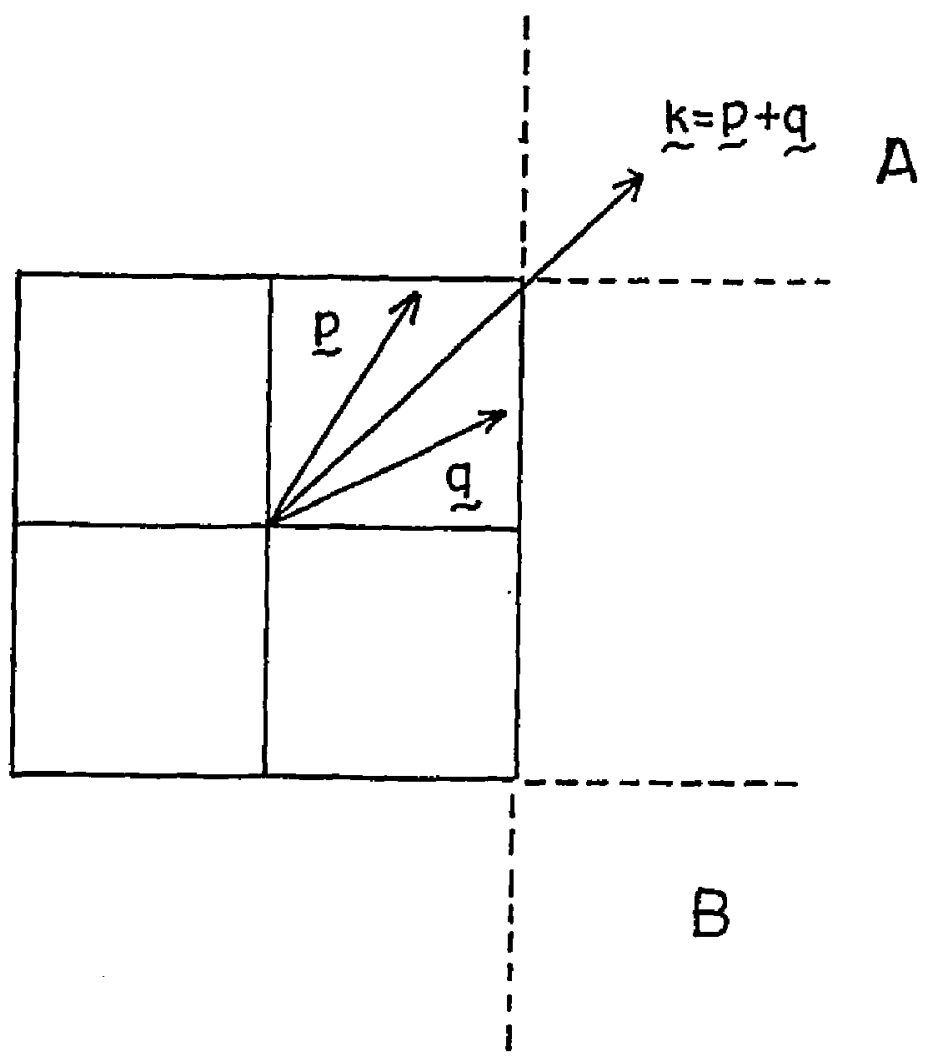


If $\underline{k}=\mathrm{p}+\mathrm{g}$ lie in region $\mathrm{A}$ or $\mathrm{B}$ then double aliasing occurs. It seems very reasonable to perform isotropic truncation for isotropic turbulence simulation. This can be done in such a way that the modes that cause double aliasing are themselves zero. More precisely we set

$$
\begin{aligned}
& u(\underline{k})=0 \\
& v(k)=0
\end{aligned}
$$

$$
\text { if }|k|>\alpha \mathrm{N} / 2
$$

where $a$ has to be determined. We want the largest $a$ for which

$$
\begin{aligned}
& \sum_{k=\mathfrak{p}+q+N} u(\mathfrak{q}) v(g)=0 \\
& |\mathfrak{p}|<a \mathrm{~N} / 2 \\
& |\mathrm{~g}|<a \mathrm{~N} / 2 \\
& |\mathfrak{k}|<a \mathrm{~N} / 2
\end{aligned}
$$

where $N=(N, N)$. That is, we look for the smallest value of $\alpha$ for which the following conditions are satisfied.

$$
\begin{aligned}
& |\mathrm{g}| \leqslant a \mathrm{~N} / 2 \\
& |\mathrm{~g}| \leqslant a \mathrm{~N} / 2 \\
& |\mathrm{~g}| \leqslant a \mathrm{~N} / 2 \\
& k=\mathrm{D}+\mathrm{g}+\mathrm{N}
\end{aligned}
$$

This optimization problem was solved by Patterson and Orszag (1971), who supplied a table of values of a as a function of N. $a$ is .975 and .95 for $\mathrm{N}=32$ and 64 respectively. 
TIME INTEGRATION

The dynamical equations that are advanced in-time may be written schematically as

$$
\frac{d Q(k)}{d t}=P(Q(k))
$$

Here $Q(k, t)$ stands symbolically for the Fourier coefficients of all the fields. The time stepping scheme we have used is

$$
\begin{aligned}
& Q^{n+1 / 2}(\underset{\sim}{k})=Q^{n}(\underset{\sim}{k})+P\left(Q^{\mathfrak{n}}(\underset{\sim}{k})\right) \Delta t / 2 \\
& Q^{n+1}(\underset{\sim}{k})=Q^{n}(\underset{\sim}{k})+P\left(Q^{n+1 / 2}(\underset{\sim}{k})\right) \Delta t
\end{aligned}
$$

The index $n$ indexes the $n$th time step of duration $\Delta t$. This method is of second order accuracy. The error at any one time step is of order $(\Delta t)^{3}$. For a fixed time of integration total number of time steps required varies as $1 / \Delta t$. Therefore the cumulative error is of order $(\Delta t)^{2}$.

Linearized analysis of numerical stability does not predict non-1inear stability but gives us valuable ideas about the scheme. We calculate amplification of errors due to time discretization for the one-dimensiona1 Navier-Stokes case (Potter 1972).

$$
\frac{d \omega}{d t}=v \frac{d \omega}{d x}
$$

We 1 inearize by assuming $v=$ constant $=U_{0}$ 
Then

$$
\begin{aligned}
& \frac{d \omega}{d t}=-i k U_{0} \omega \\
& \omega^{n+1 / 2}=\omega^{n}+\left[-i k U_{0} \omega^{n}\right] \Delta t / 2 \\
& \omega^{n+1}=\omega^{n}+\left[-i k U_{0}\left(\omega^{n}-i k U_{0} \omega^{n} \Delta t / 2\right)\right] \Delta t
\end{aligned}
$$

Therefore the amplification factor $g$ (ratio of the error in the $k$ th component at $n$th time step to that at the preceding time step) is given by

$$
|\mathrm{g}|^{2}=1+1 / 4\left(\mathrm{kU}_{0} \Delta t\right)^{4}
$$

This means that the scheme is asymptotically unstable, i.e. the solution will blow up as time goes to o no matter how small $\Delta t$ is. But since we are interested in intograting only up to a finite time we can always keep the error growth for a given spatial discretization within tolerance by adjusting $\Delta t$. For our case $k_{\max }=16, \Delta t=1 / 256$ and $v_{0} \sim 1$ Therefore

$$
\lg l_{\max }=1+3.8 \times 10^{-6}
$$

For $n$ time steps, error amplification is

$$
\left.\lg \right|^{n}=(1+x)^{n / 2}
$$

where $x=1 / 4\left(\mathrm{kU}_{0} \Delta t\right)$

Therefore $|g| n<\exp (x n / 2)$. 
If we integrate up to a time $T$ for which there will be an e-fold error propagation, then

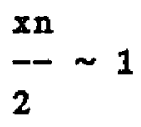

i.e.

$$
T \sim \frac{8}{\left(k U_{0}\right)^{4}(\Delta t)^{3}}
$$

Fox our case $T \sim 2048$ while we integrated only up to $T=274$ at the most Therefore even though the scheme is unstable it is good enough for our case.

This analysis had been for inviscid case. If we consider the effect of just the viscous term we will have

$$
g=1-\Delta k^{2} \Delta t\left(1-\nabla k^{2} \Delta t / 2\right)
$$

which implies $\Delta t$ has to be less or equal to $2 / \mathrm{s}^{2} \max$ for the scheme to be numerically stable. For all the reported runs we have used $\Delta t=1 / 256$ and $2 / 2 k^{2} \max$ was 3.9

We have studied the effect of varying $\Delta t$ by cutting it in half for a 5000 time steps-segment and found that the two solutions agreed well.

FORCING ALGORITIM

The study of cascades involves supplying excitations to the system continuously. Physically if we stir a magnetofluid with a conducting stirrer then the systom is supplied with mechanical and magnetic energy 
at wave numbers reciprocal to the scales of motion of the stirrer. Even though cascade predictions assumed constant rate of supply of energy and enstrophy or energy and mean square vector potential, it is much easier to supply excitations keoping the forcing amplitude constant. The forcing function $F$ and $G$ satisfies the following difference equation.

$$
F_{n+1}=f F_{n}+\left(1-f^{2}\right)^{1 / 2} J_{n+1}
$$

$f$ is a memory fraction $\leqslant 1$ and $J_{n}$ are random numbers taken from a random number generator. Since the random numbers are uncorrelated we have

$$
\bar{F}_{n}^{2}=\text { constant }=\left\langle J_{n}^{2}\right\rangle
$$

We chose the random numbers from a gausian random number generator with zero mean and unit variance. 
Chapter III

CASCADES AND INVERSE CASCADES

The idea of a cascade was first introduced for the Navier-Stokes fluid (Kolnogoroff 1941). The Navier-Stokes equation can be written in Fourier-Transformed space as:

$$
\frac{d u_{i}(k, t)}{d t}=-\frac{i}{2} p_{i j k}(k) \sum_{p^{+g=k}} u_{j}(p, t) u_{k}(g, t)-\nu k^{2} u_{i}(k, t)
$$

where

$$
p_{i j k}(\underline{k})=k_{j}\left(\delta_{i k}-k_{i} k_{k} / k^{2}\right)+k_{k}\left(\delta_{i j}-k_{i} k_{j} / k^{2}\right)
$$

The first term on the right is the non-linear term and couples triad of Fourier modes which form a triangle. Because of the non-1inear term modes that are not excited initially can be excited later in time.

The most important quantity in cascade theory is the energy spectrum $E(k, t)$ which evolves in time according to the following equation.

$$
\overline{d E}_{d t}^{d k, t)}=F(k, t)-2 \nu k^{2} E(k, t)
$$


The first term on the right represents transfer of energy between wave numbers. This is justified since

$$
\int_{0}^{\infty} F(k, t) d k=0 .
$$

The second term on the right represents viscous dissipation and is stronger at higher $k$.

The non-1inear term spreads energy in wavenumber space. The process is checked by viscous damping at large wavenumber. At the other end of k-space there are always larger scales where there is no energy. Therefore the spectrum function will have a maximum at some value of $k$ and fall of to zero as $k$ goes to infinity. There is experimental evidence for this conjecture (Tritton 1977, Batchelor 1959). Some part of the spectrum may be in approximate statistical equilibrium. This means that the rate of change of mean values (of say, energy in a particular wavenumber) is negligible. As the Reynolds number increases inertia forces dominate over viscous forces up to higher and higher wave number. The energy sink goes away to a higher wavenumber.

At the early stages of turbulence only a few wave numbers are excited as is done in an initial value problem. The action of inertia transfers energy to other (in general higher) wavenumbers. U1timately energy will dissipate at the sink provided by viscosity. The wave numbers, adjacent to the ones that are directly affected by the external agent, are affected less strongly by the agent. The influence reduces at the far end of the chain of transfer process. There will be loss of influence dur- 
ing this transfer process. For example, the directional preference, if any will ultimately disappear. In other words we can hypothesize that distant wavenumber modes are statistical1y independent.

The ravenumber beyond which viscous dissipation plays an important role may be marked as $k_{d}$ - the dissipation wave number. This may be the wave number at which the dissipation of energy is a maximum. If the energy containing eddies are far below $k_{d}$ then we have a range of wavenumbers where viscosity is irrelevent. Taylor (1938) showed that in this range the decay of energy is independent of viscosity. The rate of decay is identical with the rate at which energy is transferred by inertia forces. Energy containing eddies determine the rate of energy transfer and high wavenumbers adjust themselves according to the Reynolds number. Higher wavenumbers are statistically independent of lower wavenumbers. Therefore high wavenumber modes must be in equilibrium in the statistical sense.

The equilibrium or inertial range is internally self adjusted through the operation of inertia forces. This also depends on external parameters via the rate of energy supply $e$ which is also equal to the rate of removal of energy at the other end by dissipation. The dissipative loss at the equilibrium range is distributed over the whole range which depends in a way on viscosity.

If we choose $\supset$ and $\varepsilon$ to construct dimensionally length ( 1 ) and velocity $(v)$ then $1=\left(v^{3 / \varepsilon}\right)^{1 / 4}$ and $v=(\Delta \varepsilon)^{1 / 4}$ (Batchelor 1953). Then the motion will have a universal statistical form. More precisely, the joint probability distribution of values of Fourier Coeffients of veloc- 
ity at different values of $b$ is independent of time, isotropic in form and universal.

The notion is uniquely detornined when the rate of energy supply $\varepsilon$, to the inertial range, is specified. Ilaving a universal equilibrium hypothesis we can predict statistical quantities that depend on1y on Fourier coefficients. For exanple the spectrun function $E(k)$ can be predicted. It should take a universal form such that the paraneter disappears from the cxpression for the mean values.

Therefore if we choose a conbination of $k$ and $\varepsilon$ to form $E(k, t)$ the only dimensional1y correct form is $E(k, t)=C \varepsilon^{3 / 2} \mathrm{k}^{-5 / 3}$.

In this range energy transferted to a wave number from below is equal to energy transferred fron that wave number to the ones above it. Pictorially (Kraichnan 1967):

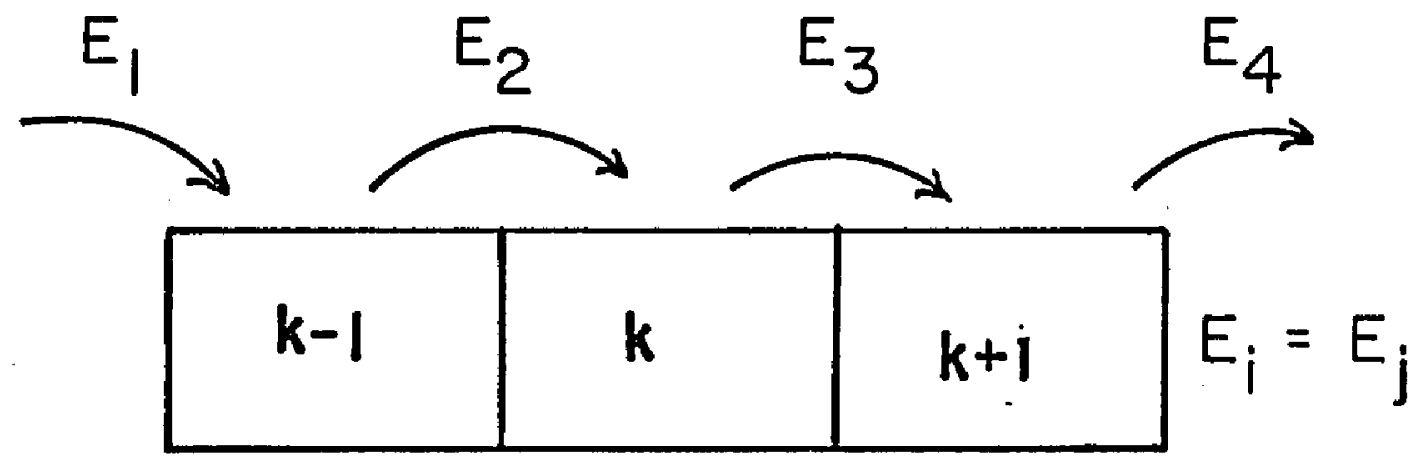

N11 these results are for the three-dinensional Navier-stoles fluid. In tro-dimensional llavier-Stokes fluid there are tro inviscid constants of hotion: marely, the energy and the enstrophy (nean square vorticity). 
Fjørtoft (1953) pointed out that two constants of motion imply that any transfer of energy to higher wavenumbers must be accompanied by a bigger transfer to lower wavenumbers. So in $2 \mathrm{D}$ we can not reduce a triad interaction into pair interaction as in the figure above, because pair interaction can not conserve both energy and enstrophy. In these situations a dual cascade is proposed. There can be a range with zero enstrophy and finite energy flux and another range with zero energy and finite enstrophy flux.

If the energy cascade rate is $\varepsilon$, which is independent of $k$, then the enstrophy cascade rate in the same range must have the form $\eta=A^{2} \varepsilon$ with $A$ independent of $k$. But $\eta$ itself must be $k$-independent. Therefore A must be equal to zero implying zero enstrophy flux, $\eta=0$. That is the enstrophy cascade is zero where $z$ is independent of $k$. Similarly energy f1ux is zero where $\eta$ is independent of $k$. Therefore there must be two different inertial ranges for the two quantities to cascade- a dual cascade situation (Kraichnan 1967).

Hith a Kolmogorof style dimensional analysis we will have the power laws for the omni-directional spectrum function:

$$
\begin{aligned}
& E(k)=C \varepsilon^{2 / 3} k^{-5 / 3} \quad \text { for energy cascade, } \\
& \text { and } E(k)=C_{\eta}{ }^{2 / 3} k_{k}^{-3} \quad \text { for enstrophy cascade. }
\end{aligned}
$$

In two-dimensional MHD with $\mathcal{\nu}=0=\mu$ we have three constants of motion. The important two are total energy $E$ and mean square vector potential A. The third one is cross helicity which may be zero for uncorrelated mechanical and magnetic forcing. A dual cascade may be 
hypothesized. Kolmogor of dimensional analysis leads to the following power law for the vector potential spectrum (Again omni-directional).

$$
\begin{array}{ll}
\mathrm{a}(\mathrm{k})=\mathrm{C} \varepsilon^{2 / 3} \mathrm{k}^{-11 / 3} & \text { for energy cascade, } \\
\text { and } \mathrm{a}(\mathrm{k})=\mathrm{Ca}^{2 / 3_{\mathrm{k}}-7 / 3} & \text { for vector potential cascade. }
\end{array}
$$

If energy is supplied to a fluid or magneto-fluid in a band of wavenumbers then there can be two inertial ranges on two sides of the forcing band. On one side a cascading quantity cascades having zero flux of the other and on the other side the second quantity cascades with zero flux of the first quantity.

Now the question is which of the two quantities cascades in which direction? There are two hints to the answer. First, the quantity which in the non-dissipative equilibrium theory can be sharply peaked at sma11 $k$ is the one which may cascade to longer wave lengths (inverse cascades). In the Navier-Stokes case it is energy and in lHD it is the mean square vector potential. Second, quantity which contains higher powers of $k$ in its defining equation may cascade to high $k$ (direct cascades), as it is easier to dissipate at high $k$. In the Navier-Stokes case this is enstrophy and energy in the hHD case.

Analytically one can only prove that the two cascades are in opposite directions. For the Navier-Stokes case see Kraichnan (1967).

So in summary the situation is described pictorially below. 


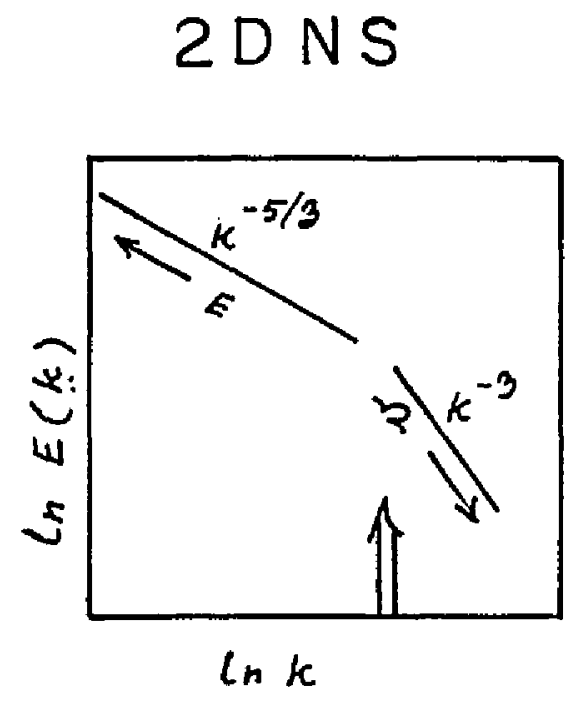

2D MHD

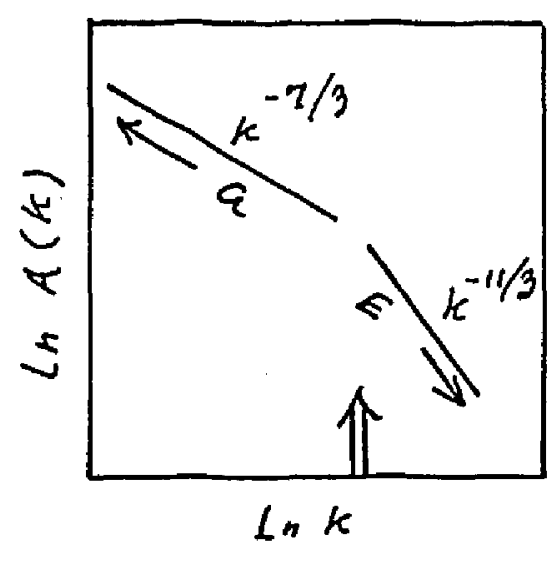




\section{Chapter IV}

\section{RESULTS}

\section{a) EQUILIBRIUH RUNS:}

Equilibrium predictions for the spectral distribution of energy, vector potential etc, have already been tested (Seyler et al 1975, Fyfe ct al $1977 a)$. Nonetheless these predictions are the only available means for testing a numerical code. We have made these non-dissipative runs precisely for this purpose.

Figure 1A shows the initial modal onergy spectrum for the inviscid un-forced Navier-Stokes run. In a1I the previous studies the initial spectra were chosen to be non-zero only in a narrow annulus of wavenumbers, normally at low $k$ (Seyler et al 1975, Fyfe et al 1977a, Shebalin et al 1983) Here we have started with a full spectrum which is taken from an instant of a forced run. The grid size was $32 \times 32$ and $\Delta t$ was 1/256. Figure 1B shows the final modal energy spectrum averaged over the last 1000 time steps for the same run. The solid curve is the equilibrium prediction calculated from the reciprocal temperatures $a$ and $\beta$. For our case $a$ and $\beta$ were -13.4 and 18.3 respectively. The final equilibrium spectrum is peaked at the fundamental $k_{m i n}$. This is an indication that in viscous driven case energy may cascade to long wavelengths. The energy was conserved better than .2 percent and enstrophy better than 2 percent. 
For the MIID case wo again have started with full spectra of both mechanical and magnetic energies. These spectra were taken from an instant in the forced MHD run. We have used a $32 \times 32 \mathrm{grid}$ and $\Delta t=1 / 256$ also in this case.

Figure $2 A$ and $2 B$ are the initial spectra for the mechanical modal energy and vector potential respectively. Figure $2 \mathrm{C}$ and $2 \mathrm{D}$ are the final spectra for the same two quantitios respectively and are averaged over 2000 time steps. Solid curves are the equilibrium predictions with reciprocal temperatures $\alpha=714, \beta=0$ and $\gamma=-446$. The final equilibrium vector potential spectrum shows that it is peaked at the lowest $k$, an indication for inverse cascade in dissipative driven run. Mean square vector potential and total energy were conserved, better than .3 and .2 percent respectively.

In absence of time discretization and round-off error these quantities should be conserved exactly, independent of k-space truncation. Therefore the lovel of agreement is a measure of error.

b) DISSIPATIVE FORCED NAVIER-STOKES RUN:

For the Navier-Stokes(NS) run we have started with an empty spectrum and drive it with a random forcing according to the prescription described in Chapter II. We have again used a $32 \times 32$ grid and the Reynolds number equal to 500. The forcing band was between $\mathrm{k}_{\mathrm{Fmin}}{ }^{2}=55$ and $k_{\text {Fmax }}{ }^{2}=70$.

Figure 3A shows the total energy 


$$
E(t)=\sum_{k} E(\underline{k})=1 / 2 \sum_{\underline{k}}|y(k, t)|^{2}
$$

as a function of time. The two most significant features of the curve are: i) the total energy increases systematically with time, as is expected for an inversely cascading quantity, ii) the total energy appears to be approaching asymptotically to a constant value, whereas for an unbounded system in the limit of infinite Reynolds number a linear increase of $E(t)$ with $t$ might be expected. As will be discussed in Chapter $V$, the curve in figure $3 A$ is rather well fit by the expression $E=E_{\max }[1-\exp (-t / \tau)]$ with $E_{\max }=3.9$ and $\tau=124$.

Also in figure $3 \mathrm{~A}$, we show as squares, the energy contained in the fundamental $k_{\min }{ }^{2}=1$. It will be apparent that by $t=30$, it already accounts for a significant fraction of the total.

Figure 3B shows the total enstrophy

$$
\Omega=\sum_{k} \Omega(k)=1 / 2 \sum_{k}|\omega(k, t)|^{2}
$$

versus time. The enstrophy should be the directly cascading quantity, and reaches a value about which it fluctuates while the energy continues to grow.

Figures $4 \mathrm{~A}, 4 \mathrm{~B}$ and $4 \mathrm{C}$ show the time averaged modal energy spectra $|y(k)|^{2}$ versus $k^{2}$. These axe averaged over all the values of $\underset{\sim}{k}$ corresponding to a given $\mathrm{k}^{2}$. Figure $4 \mathrm{~A}$ shows a relatively early spectrum averaged over 500 time steps ending at $t=19.53$. Figure $4 B$ is the modal energy spectrum averaged over 1000 time steps ending at $t=29.3$, which is 
about the time when the fundamental is almost at par with the rest of the spectrum. Figure 4C is the same at the end of the run, at $t=273.44$. (Every fourth point is plotted for $k^{2} \geqslant 70$, to avoid cluttering the graphs.)

The crucial result of this study is thought to be illustrated by the fact that the fundamental has come to contain about ninety percent of the total energy.

In figure 4C we also have shown a least square fit of a power law for the two inertial ranges. The exponents turned out to be -1.16 and -1.62 respectively while Kolmogoroff dimensional analysis predicts them to be -1.33 and -2.0 respectively.

Figures 5A, $5 B$ and $5 C$ show the contours of constant stream function at three stages of the simulation. These figures display how the large scale stream function evolved to fill up the whole box. The dotted contours represent negative values of the stream function.

Figures $5 \mathrm{D}, 5 \mathrm{E}$ and $5 \mathrm{~F}$ show the contours of constant vorticity at times $t=19.53,97.66$ and 273.44 respectively. Agatin the broken curves represent negative values. Vorticity contours have more small scale structures than the contours of stream function since vorticity contains nore powers of $k$ in its defining expression in wavenumber space.

Figures $6 \mathrm{~A}$ through $6 \mathrm{~J}$ show the modal energy at different values of $\mathrm{k}^{2}$ as a function of time. All these modes grew to constant values about which they fluctuate. These should be contrasted with the continued growth of the fundamental displayed in figure $3 A$. These fluctuations 
can be traced quite closely. There are on the average a few hundred time steps between two peaks.

Figure 7 exhibits the Carnevale (1981) entropy

$$
S=\sum_{k} \ln |x(k, t)|^{2}
$$

as a function of time. This function is analogous to the Boltzman IIfunction in gas dynamics. The function quickly maximizes and then gradually decays. The interpretation of the decay might loosely be that as the system becomes dominated by the fundamental, the effective degree of randomness decreases. (The action of viscosity is typically to decrease entropy). This entropy has its origins in information theory, and descends from the Burg entropy (Burg 1975).

To see how severe the truncation at the high end of the wavenumber space was, we look at the enstrophy dissipation wavenumber defined by

$$
k_{\mathrm{d}}=\left(\eta / \nu^{3}\right)^{1 / 6}
$$

where $\eta$ is the average enstrophy per unit time supplied to the fluid. The enstrophy supply rate may be estimated from the relation $\eta=\mathrm{k}_{\mathrm{F}}{ }^{2} \varepsilon$ where the energy supply rate $\varepsilon$ is given by $2 \nu \Omega$. For the run reported $k_{d}$ was 27 at the end of the run. Since dissipation wavenumber is an order of magnitude calcnlation it is customary to accept the results to be good if $k_{d}$ is less than twice $k_{\max }$.

It is sometimes useful to define a mean wavenumber $k_{\text {mean }}=(\Omega / E)^{1 / 2}$ which indicates in which part of the wavenumber space much of the activ- 
ities are going on. For our run $k_{\text {mean }}$ was 1.96 at the end of the run. At the begining of the run $k_{\text {mean }}$ was $j u s t$ equal to the forcing wavenumber. As time goes $k_{d}$ shifts towards low wavenumber (typical of inverse cascades).

c) DISSIPATIVE FORCED MHD RUNS:

Wo will report the results for two MID runs for the dissipative forced case. For the first run we have used a $32 \times 32$ grid and both the kinematic viscosity and the magnetic diffusivity equal to .002 . The second run was performed on the Cyber 203 computer with and $\mu$ equal to .01 . We started with a $32 \times 32$ grid and then switched to a $64 \times 64 \mathrm{grid}$ (for reasons of computer budget). In both runs we have started with empty spectra and the forcing band was between $k_{F}{ }^{2}=55$ and $k_{F}{ }^{2}=70$. In both cases we have chosen the forcing terms $F$ and $G$ in such a way that the supplies of kinetic and magnetic energies were approximately of equal magnitudes. In both cases $\Delta t$ was $1 / 256$.

ci) First MHD run:

This is the longest MHD run we have done. Figures 8 to 10 are the results of this run.

$$
\begin{aligned}
& \text { Figure } 8 \mathrm{~A} \text { is a plot of total energy } \\
& E=1 / 2 \sum_{k}|\mathrm{~g}(\mathrm{k}, \mathrm{t})|^{2}+1 / 2 \sum_{\mathrm{k}}\left[\left.\mathrm{B}(\mathrm{k}, \mathrm{t})\right|^{2}\right.
\end{aligned}
$$


versus time. The total energy being the directly cascading quantity rises to a constant value and fluctuates about that value. On the same plot we have also displayed the mechanical and magnetic contributions labeled as ' $V$ ' and ' $B$ ' respectively. The magnetic contribution is slightly higher than the mechanical one.

Figure $8 \mathrm{~B}$ shows the mean square vector potential

$$
A=1 / 2 \sum_{5}|A(k, t)|^{2}
$$

as a function of time with squares illustrating the contribution of the fundamenta1. Mean square vector potential is the inversely cascading quantity and hence should increase systematically which is what is happening here. We believe that if the system is driven long enough so that the fundamental will have enough magnetic excitations to be checked by its own dissipation rate, only after such a long time the curve would leveI off.

Figure $9 \mathrm{~A}, 9 \mathrm{~B}$ and $9 \mathrm{C}$ are the vector potontial spectra at different times of the run. Figures $9 D, 9 E$ and $9 F$ are the mechanical modal energy spectra at the same three stages of the run. Figures $9 G$, $9 H$ and $9 I$ are the magnetic modal energy spectra. Figure 91 shows least square fit to the data the expression of the form

$$
\mathrm{B}(\mathrm{k})=\mathrm{Ck}^{\mathrm{n}}
$$

for the two cascading regions. Figures $9 \mathrm{C}$ and $9 \mathrm{I}$ display the fundamental dominated spectra for the magnetic exitations. 
The least square fit to the power 1 aw gives $n=-.11$ and -0.48 for the inverse and direct cascades (Figure 9I). These values are far away from the Kolmogoroff exponents which are -.667 and -1.33 respectively (For modal energies). The insufficiency in spatial resolution manifested in its worst in the MHD runs. We need the ratio of $k_{\max }$ to $k_{\min }$ to be at least 1000 to see all ranges resolved. We are no where close to that. A dissipation wavenumber may be defined, for a situation in which total energy cascades to high $k$, in the following way.

$$
k_{d}=\left(\frac{2 \Omega}{\nu^{2}}+\frac{2 J}{\mu^{2}}\right)^{1 / 4}
$$

where $\Omega$ and $J$ are kinetic enstrophy and mean square current respective1y. For this run $k_{d}$ was 75 while $k_{\max }$ was only 16 . This is the reason why we did another run with dissipation wavenumber resolved. The results of which are given in section (cii).

Figures $10 \mathrm{~A}$ through $10 \mathrm{~L}$ are the contour plots of stream function, vector potential, vorticity and current density at three different times of the run. Vector potential contours are of large scale but not as large as the stream function contours in the Navier-Stokes run. Quantities having higher powers of $k$ in their defining expressions in $k$-space have more small scale structures.

cii) Second MHD (Star) Run:

Figure 11A shows the plot of total energy as a function of time. Again the mechanical and magnetic contributions are labeled as ' $V$ ' and 
' $B$ ' respectively. The large values of magnetic energy are typical of forced MHD runs. The arrow on the time-axis shows the time at which the grid size was changed.

Figuro $11 \mathrm{~B}$ is the plot of mean square voctor potential versus time, with squares illustrating the contribution due to the fundamental. Again the arrow shows the time when the mesh was refined.

The vector potential, mechanical and magnetic modal energy spectra are shown in figures $12 \mathrm{~A}, 12 \mathrm{~B}$ and $12 \mathrm{C}$ respectively. They are averaged over 50 values, spaced 100 time steps apart, ending at $t=97.66$. This is the time after which we have switched to $64 \times 64$ grid. The same three spectra at a later time $t=117.19$ are shown in figures $12 \mathrm{D}, 12 \mathrm{E}$ and $12 \mathrm{~F}$ (64×64). This time they are averaged over 25 values. Both of these plots display fundamental dominated spectra. Figure 12F shows least square fit to the data expressions of the form $B(k)=C_{k}{ }^{n}$ with $n=-.23$ and $-2,2$ in the two inertial ranges respectively.

Figures 13A through 13D show the contours of strean function, vector potential, vorticity and current density at $t=117.19$.

The dissipation wavenumber for this run was 33 both before and after switch .

In summary, we have demonstrated in both 2D NS and 2D MHD cases that the final state is the one which is dominated by the fundamental. 
d) PHASE RANDOHTZED RUN (NAVIER-STOKES):

An obvious question which has no simple answer is whether there is a sense in which the code can be called accurate after the many eddy turn over times represented in these runs. Accuracy checks for such problems are few, and mostly consist of inferences drawn from conservation laws of the ideal invariants ('rugged invariants') which survive the limitation to a finite number of Fourier coefficients. The fact is that the code will not conserve these invariants out to the times we are reporting. These long times are dictated by the physics of the situation (it takes that long for the fundamental to come to dominate) and there are no conclusive statements about the accuracy that we can make.

He can, however, derive considerable reassurance from the following observations. Except for the very beginning ( $t<5$ say) there are no periods of time in which large qualitative changes occur in the behavior of the computed quantities. The code is accurate (in the above sense i.e. conservation of inviscid invariants) over this initial time interval, and after that, there is only a slow, systematic evolution, always in the same direction. The code can be re-started and run accurately over any slice of time during the evolution after randomizing the phases of the Fourier coefficients: e.g. $\omega\left(\frac{k}{\sim}\right)-\omega(k) \exp (i \emptyset)$ at some instant, where $\emptyset$ is a random number between $-\pi$ and $\pi$.

Figure $14 A$ shows the results of such a typical restart at $t=58.6$ for the 2D NS run, in a plot of $E(t)$ versus $t$. The two sets of random numbers are the same. There is no noticeable departure of the two solutions from each other, even after 10000 time steps. 
Plotted in figure 14B are time averaged modal energy spectra for the two runs, over 1000 time steps onding at $t=97.66$. Again, no noticeable difference appears. 


\section{Chapter V}

\section{ALGEBRAIC MODEL FOR THE FINAL STATE}

The indications are, for the runs reported in chapter IV, that a steady state is being approached in which the fundamental dominates the spectrum, and the transfer to it is balanced by its own dissipation. This has led us to try an algebraic model of the dynamics of this process.

Once the fundamental has begun to dominate, most of the new energy goes into the fundamental, so that

$$
\begin{aligned}
& E=E\left(k_{m i n}\right) \\
& \text { and } \frac{d E}{d t}=\frac{d E\left(k_{m i n}\right)}{d t}
\end{aligned}
$$

We assume that the total enstrophy has become constant, so that

$$
\frac{d \Omega}{d t}=0 .
$$

A significant fraction of the enstrophy may be lodged in the values of k) $k_{\min }$. As the Reynolds number increases this conclusion will become wore accurate. Let

$$
\begin{aligned}
& \qquad=\Omega^{\prime}+\Omega\left(k_{\min }\right) \\
& \text { where } \Omega^{\prime}=\sum_{k>k_{\min }} \Omega(k) \\
& -41-
\end{aligned}
$$


The evolution of the energy and enstrophy are now modelled by

$$
\begin{aligned}
& \frac{d E}{d t}=-2 \vartheta \Omega+f_{E} \\
& \frac{d \Omega}{d t}=-2 \downarrow j k_{k}^{2} \Omega(k)+k_{F}^{2} f_{E}
\end{aligned}
$$

where $f_{E}$ is the rate of supply of energy by $F$ and $k_{F}$ is a wavenumber at the center of the forcing band.

$$
\begin{aligned}
& f_{E} \text { may be eliminated from equation }(5.1) \text { and }(5.2) \text { to give } \\
& \frac{d E}{d t}=-2 \nu \Omega+\frac{2 \nu}{\mathrm{k}_{\mathrm{F}}{ }^{2}} \underset{\approx}{\mathrm{k}} \mathrm{k}^{2} \Omega(\mathrm{k})
\end{aligned}
$$

Now noticing that $k_{\text {min }}=1$ this becomes

$$
\frac{d E}{d t}+2 \nu\left(1-\frac{1}{-) E}=-2 \nu \Omega^{\prime}+\frac{2 \nu}{k_{F^{2}}} \sum_{F^{2}} \sum_{k>k_{\min }} k^{2} \Omega(k)\right.
$$

The right hand side of equation (5.3) can be evaluated from the spectrum at the end of the run. The result is that equation (5.3) becomes of the form

$$
\frac{d E}{d t}+\frac{1}{\tau} E=\frac{E_{\text {max }}}{\tau}
$$


The right hand side of equation (5.3) may also be evaluated in terms of the spectrum $|x(k)|^{2}=C_{1} k^{-n 1}$ for $k$ below the forcing band and $c_{1} k^{-n 2}$ above it, with $\mathrm{n} 1, \mathrm{n} 2$ and $\mathrm{C}_{1}$ taken from the computed values in figure 4C. The two parameters in equation (5.4) thus calculated are $E_{\max }=3.58$ and $\tau=254$.

The solution $E=E_{\max }(1-\exp (-t / \tau))$ which fits the shape of figure $3 \mathrm{~A}$ rather well has $E_{\max }=3.9$ and $\tau=124$. So we can say that the model-fit was not too bad but it might be, in the high Reynolds number limit, that this simple model in which the energy dissipation occurs mainly in the fundamental and balances the input, could be sharpened considerably.

Figure I5A shows a fit to the energy versus time curve. The one with parameters taken from data is labeled ' $D$ ' and the one with parameters from the model calculation is labeled ' $M$ '. Our claim is that with highex Reynolds number and higher spatial resolution the model will fit the numerica1 result better.

A similar model may be proposed for the MilD case. We now assume

$$
\begin{aligned}
& a=a\left(k_{\min }\right) \\
& \text { and } \quad \frac{d a}{d t}=\frac{d a}{d t}\left(k_{\min }\right)
\end{aligned}
$$

We a1so assume that the total energy has become constant. So that

$$
\frac{d E}{d t}=0 .
$$

$$
\begin{array}{ll}
\text { Now we wite } & a=a^{\prime}+a\left(k_{\min }\right) \\
\text { With } & a^{\prime}=\sum_{k>k_{\text {min }}}|A(k)|^{2}
\end{array}
$$


The evolution of total energy and mean square vector potential are given by

$$
\begin{aligned}
& \frac{d E}{d t}=2 f_{E}-\sum 2 \nu k^{2}|\underset{v}{(k)}|^{2}-\sum 2 \mu k^{2} \mid \underset{\sim}{\left.(\underset{\sim}{k})\right|^{2}} \\
& \frac{d a}{d t}=f_{E^{2}} / k_{F^{2}}-\sum 2 \mu k^{2}|A(k)|^{2}
\end{aligned}
$$

We have used a forcing algorithm such that the magnetic and velocity fields axe forced approximately with equal strength. We used $G=F / k_{F}{ }^{2}$ in the above equations.

Eliminating $f_{E}$ from these two equations and noting that $k_{\min }=1$ we have the following.

$$
\frac{d a}{d t}+2 \mu\left(1-\frac{1}{2 k_{F^{2}}}\right) a=\frac{\partial \Omega}{k_{F}{ }^{2}}+\frac{\mu}{k_{F^{2}}} \sum_{k>k_{\min }} k^{2}|B(k)|^{2}-2 \mu \sum_{k>k_{\min }}|\mathrm{B}(k)|^{2}
$$

We have used $k^{2}|A(k)|=|\underset{\sim}{B}(k)|$ in the above equation. We can again write this equation as

$$
\frac{d a}{d t}+\frac{a}{\tau}=\frac{a_{\max }}{\tau}
$$

Calculation of the right hand side provides us with $a_{\max }=.1982$ and $\tau=252$.

Figure 15B is a plot of mean square vector potential versus time. The points labeled ' $M$ ' are calculated from this model.

Figure $15 \mathrm{C}$ is the plot of $E(t)$ versus $t$ of an auxiliary run which has been done to gain better confidence in the final state predicted by the 
model. We changed the energy in the fundamental at $t=273.44$ from the NS run by replacing $\omega(k=1)$ with $\omega(k=1) \exp (i \emptyset) \times 3.0$, where $\emptyset$ is a random phase angle. From there on we let the system run as it was. We notice that the energy is decreasing asymptotically to a value. We expect that this final value to which the system relares is the one predicted by the model we proposed. 
Appendix A

NON-DISSIPATIVE PREDICTIONS

There is no physical system which is ideal, i.e. there is no real fluid with zero viscosity or magnotofluid with infinite condactivity. Still there are enough reasons for studying non-dissipative turbulence. First, the unphysical non-dissipative system obeys statistical mechanios which predicts an equilibrium distribution. The equilibrium predictions or non-dissipative constants of motion may be used to test numerical codes. Seyler et al(1975) and others have studiod purely non-dissipative cases which helped us understand many aspects which are comon to dissipative systems. The most important one is of course the action of non-linear terms. The non-dissipative constants of motion are the candidates for cascades in dissipative driven cases. The nature of equilibrium gives us a hint as to which direction what global quantity cascades. In the non-driven dissipative case the non-dissipative constants of motion and their various ratios are interesting quantities for studying selective decays (Mathaeus 1980).

\section{i) NAVIER-STOKES CASE}

Energy per unit volume is a constant of motion for incompressible non-dissipative Navier-Stokes fluids. The proof is the following.

$$
\frac{d E}{d t}=\frac{d}{d t} \int \frac{1}{2} v^{2} d^{3} x
$$




$$
=\int x \cdot \frac{\partial y}{\partial t} d^{3} x
$$

Using the Navier-Stokes equation we get

$$
\frac{d E}{d t}=\int x \cdot\left[-\underline{v} \cdot \nabla y-\nabla p+\nu \nabla^{2} y\right] d^{3} x
$$

Using vector identities, performing some integration by parts and using the incompressibility condition we get the following:

$$
\frac{d E}{d t}=-2 \downarrow \Omega
$$

where $\Omega$ is the enstrophy defined as:

$$
\Omega=\int \omega^{2} / 2 d^{3} x
$$

Therefore for non-dissipative fluids, i.e. with $\nu=0$, we get conservation of energy.

In two-dimensions we have an infinite set of constants of motion, namely

$$
I_{n}=\int \omega^{n} d^{2} x
$$

To prove this we start with the definition of $I_{n}$.

$$
\underset{d t}{d t}=\int n \omega^{n-1} \frac{\partial \omega}{\partial t} d^{2} x
$$

Using the Navier-Stokes equation to substitute for $\partial \omega / \partial t$, we get

$$
\frac{d I_{n}}{d t}=\int n \omega^{n-1}(-y \cdot \nabla \omega) d^{2} x
$$




$$
\begin{aligned}
& =-\int x \cdot \nabla\left(\omega^{n}\right) d^{2} x \\
& =-\int d^{2} x \nabla \cdot\left(y \omega^{n}\right) \\
& =0
\end{aligned}
$$

He have used the incompressibility condition $\nabla \cdot y=0$. The last step follows if we assume periodic boundary conditions or no-silp conditions. One of these constants of motion is enstrophy, namely the quadratic one. Energy and enstrophy can be written in wavenumber space as

$$
\begin{aligned}
& \mathbb{E}=\sum|\mathrm{x}(\mathrm{k})|^{2} \\
& \Omega=\sum \mathrm{k}^{2}|\mathrm{y}(\mathrm{k})|^{2}
\end{aligned}
$$

The two quantities are not only constants globally, they are invariants individually by each triad of interacting wave vectors $(\underline{+k}, \pm \underline{\underline{k}}, \pm g)$ (Kraichnan 1973). This detailed conservation property imp1ies that enexgy and enstrophy are conserved even if the dynamical system is truncated by removing from all sums every wave vector whose magnitudes do not lie between wavenumbers $k_{\min }$ and $k_{\text {max }}$. Invariants that survive truncation are referred to as rugged invariants. All $I_{n}$ 's except for n=2 (i,e, enstrophy) are not rugged.

The most important property of the non-dissipative case is the existence of a Liouville theorem. Lee (1952) showed that

$$
\frac{\dot{a}_{i}(k)}{\partial a_{i}(k)}+\frac{\partial \dot{b}_{i}(k)}{\partial b_{i}(k)}=0
$$


Where $a_{i}(k)$ and $b_{i}(k)$ are respectively the real and imaginary parts of $u_{i}(k)$. This detailed Liouville theorem continues to hold if the system is truncated with limit $k_{\text {min }}$ and $k_{\max }$. It thon follows that any probability density in this divergence free phase space, which is a function of only the constants of motion, will itself be an invariant. The most important one is of course the equilibrium Gibb's ensemble.

$P \alpha \exp (-\alpha E-\beta \Omega)$

with $\alpha$ and $\beta$ playing the role of inverse temperatures. The normalization constant may be found by demanding

$$
\int P d X=1
$$

where integration is over the whole phase space, i.e. over real and imaginary parts of $v_{i}(k)$ for all $i$ and allowed wave vectors. We must not forget about the two important constraints, namely the reality condition $y(-\underline{w})=y^{*}(k)$ and the incompressibility condition $\underset{\sim}{k} \cdot \underline{\underline{w}}(\underset{\sim}{)})=0$. The reciprocal temperatures $\alpha$ and $\beta$ are chosen to satisfy

$$
\begin{aligned}
& \int \operatorname{dXP}\left[u^{2}{ }_{i R e}(\underline{N})+u^{2}{ }_{i m}(\underline{k})\right]=E \\
& \int \operatorname{dXPk}^{2}\left[u^{2}{ }_{i R \theta}(\underline{k})+u^{2}{ }_{i m}(\underline{k})\right]=\Omega
\end{aligned}
$$

Knowing this equilibrium ensemble we can predict averages of interesting quantities. For example the spectral density can be predicted to be the following (Seyler et al 1975).

$$
\left\langle|\mathfrak{n}(k)|^{2}\right\rangle=\frac{1}{a+\beta k^{2}}
$$


50

This particular two-temperature statistics has interesting unusual properties. For details we may refer to Kraichnan (1967, 1975), Seyler et al (1975), and Kraichnan and Montgomery (1980).

ii) MIID CASE

For the non-dissipative magnetohydrodynamic case we again first of all look for the constants of motion and then see how many of them are rugged.

We start with the equations that describe the time evolution of dynamica1 variables.

$$
\begin{aligned}
& \frac{\partial \omega}{\partial t} \quad=-\mathrm{x} \cdot \nabla \omega+\mathrm{B} \cdot \nabla j \\
& \frac{\partial a}{\partial t} \quad=-\mathrm{y} \cdot \nabla a
\end{aligned}
$$

By multiplying these equations by $\omega^{*}(\underset{\sim}{)})$ and $a^{*}(\underline{k})$ respectively and summing over all $\mathrm{k}^{\prime} \mathrm{s}$ we can show that

$$
\begin{aligned}
& \frac{d}{d t}\left[\sum_{2}^{1}-\left(|v(\underline{k})|^{2}+|B(\underline{k})|^{2}\right)\right]=0 \\
& \left.\frac{d}{d t}\left[\sum a^{*}(\underline{k}) \cdot \omega(\underline{k})\right]\right)=0 \\
& \text { d }\left[\sum|a(k)|^{2}\right]=0 \\
& \text { de }[\mid
\end{aligned}
$$


That is, the constants of motion are energy $E$, cross helicity $P$ and mean square vector potential $A$. These are the only rugged invariants so far known. The existence of other rugged invariants cannot be formally disproved. However, the equilibrium predictions derived from these throe invariants agree well enough with numerical solution that we can claim the non-existence of any other rugged invariants. We may refer to Kraichnan and Montgomery (1980), Fyfe and Montgomery (1976) and Fyfe et a1 $(1977 \mathrm{a})$.

By transforming the two dynamical equations in wave number space and by direct differentiation one can show the following result (Shebalin 1982).

$$
\begin{aligned}
& \frac{\dot{\partial \omega(k)}}{\partial \omega(k)}=0 \\
& -\dot{\partial a(k)} \\
& \frac{\partial a(k)}{\partial a(k)}=0
\end{aligned}
$$$$
\text { and }
$$

That is there exists a detailed Liouville theorem. Following similar arguments as Kraichnan (1975), Fyfo et al (1976), Kraichnan and Montgomexy (1980) we can describe the system by a canonical distribution function.

$D \alpha \exp (-\alpha E-\beta P-\gamma A)$

Again the normalization constant is obtained by requiring that

$$
\int D d x=1
$$

where

$$
\mathrm{dX}=\prod_{k}{ }^{\omega_{R e}}{ }^{(k){ }^{\omega}}{ }_{I m}(k){ }^{a_{R e}}(k) a_{I m}(k)
$$


Using this distribution function Fyfe et a1 (1976) showed that the spectral densities are the following.

$$
\begin{aligned}
& \left\langle|B(k)|^{2}\right\rangle=\frac{\alpha}{\alpha^{2}+\alpha \gamma / k^{2}-\beta^{2} / 4} \\
& \left\langle|v(k)|^{2}\right\rangle=\frac{\alpha+\gamma / k^{2}}{\alpha^{2}+\alpha \gamma / k^{2}-\beta^{2} / 4} \\
& \langle B(\underline{k}) \cdot v(-k)\rangle=\frac{-\beta / 2}{\alpha^{2}+\alpha \gamma / k^{2}-\beta^{2} / 4}
\end{aligned}
$$

These predictions have been verified numerically by fyfe et al (1976a). As in the Navier-Stokes case the spectral densities give us hints as to which direction which global quantity cascades. 


\section{REFERENCES}

Batchelor, G.K.(1946). The theory of axisymetric trubulence, Proc. Roy. Soc. A, 186, 480 .

Batchelor, G.K.(1953). The Theory of lomogeneous Turbulence, Cambridge University Press.

Batchelor, G.K.(1969). Computation of the energy spectrum in Ilomogeneous Two-dimensional turbulence, Phys. F1uids, 12, Supp1. II, 233.

Chandrasekhar, S.(1950). The theory of axisymmetric turbulence, Philos. Trans. A, 242, 557 .

Corrsin, S.(1962). Me'canique de 1a Turbulence, Paris: Centre Nationa de la Recherche Scientifique, pp 27-52.

E1iasen, E., Machenauer, B., and Rasmussen, E.(1970). On a Numerical Method for Integration of the Hydrodynamical Equations with a Spectral Representation of the Horizontal Fields, Report No. 2, Department of Heteorology, Copenhagen University, Denmark.

Fjortoft, R. (1953). On the changes in the spectral distribution of kinetic energy for two-dimensional non-divergent flow, Tellus, $\underline{5}$ ( 3 ), 225 .

Frisch, U., Pouquet, A., Sulem. P-I. and Meneguzzi, M.(1983). The dynamics of two-dinensional ideal magnetohydrodynanics, J. de Me'chanique The'orique et Applique'e, to appear. 
Fyfe, D. and Montgomery, D.(1976). High-beta turbulence in two-dimensional magnetohydrodynamics, J. P1asma Phys., 16 (2), 181.

Fyfe, D., Joyce, G, and Montgomery, D.(1977a). Magnetic dynamo action in two-dimensional turbulent magnetohydrodynamics, J. P1asma Phys., 17 (2), 317 .

Fyfe, D., Montgomery, D. and Joyce, G. (1977b). Dissipative forced turbulence in two-dimensional magnetohydrodynamics, J. P1asma Phys., 17 (2), 317 .

Gottlieb, D. and 0rszag, S.A.(1977). Numerical Analysis of Spectral Methods: Theory and Applications, Society for Industrial and Applied Mathematics, Philadelphia.

Hinze, J.(1975). Turbulence, HcGraw-Hi11, New York.

von Ka'rman, T, and Howarth, L.(1938). On the statistical theory of isotropic turbulence, Proc. Roy. Soc. A, 164, 192.

Kolmogoroff, A.N.(1941). The local structure of turbulence in incompressible viscous fluid for very large Reynolds numbers, C. R. Acad. Sci., U.R.S.S., 30, 301.

Kraichnan, R. H.(1967). Inertial ranges in two-dimensional turbulence, Phys. F1uids, 10 (7), 1417 .

Kraichnan, R. H.(1973). Helical turbulence and absolute equilibrium, $J$. F1uid Mech., $\underline{59}(4), 745$. 
Kraichnan, R. H.(1975). Statistical dynamics of two-dimensional flow, J. Fluid Mech., 67 (1), 155 .

Kraichnan, R. H. and Montgomery, D.(1980). Two-dimensional turbulence, Rep. Prog. Phys., , 43, 548.

Lee, T. D.(1952). On some statistical properties of hydrodynamical and magnetohydrodynamical fields, Quart. App1. Math., 10, 69.

Leith, C. E.(1968). Diffusion approximation for two-dimensional turbulence, Phys. F1uids, $\underline{11}(3), 671$.

Lilly, D. K.(1969). Numerical simulation of two-dimensional turbulence, Phys. F1uids Supp1. II, 12, 240.

Matthaeus, H. II. (1982). Reconnection in two-dimensions: Locatization of vorticity and current near magnetic $x$-points, Geophys. Res. Lett., $\underline{9}$ $(6), 660$.

Matthaeus, If. H. and Montgonery, D.(1980). Selective decay hypothesis at high mechanical and magnetic Reynolds numbers, Ann. N. Y. Acad. Sci.. 357, 203 .

Matthaeus, W. I. and Montgomery, D.(1981). Nonlinear evolution of the sheet pinch, J. P1asma Phys,. 25 (1), 11 .

Meneguzzi, M., Frisch, U., and Pouquet, A. (1981). Helical and nonhelical turbu1ent dynamos, Phys. Rev. Lett., 47 (15), 1060.

Monin, A. S. and Yaglom, A. M.(1975). Statistical Fluid Mechanics: Mechanics of Turbulence, vol 1 and 2 , MIT press, Massachussetts. 
Montgomery, D.(1982). Theory of hydromagnetic turbulence, Proc. 'Solar Wind Five', Woodstock, Vermont.

Onsager, L. (1949). Statistical hydrodynamics, Nouvo Cimento, $\underline{6}$ Suppl., 279.

Orszag, S.A.(1971). Numerical simulation of incompressible flows within simple boundaries. I. Galerkin (Spectral) representations, Stud. App1. Math., L (4), 293 .

Orszag, S. A. and Tang, C-M.(1979). Small scale structure of two-dimensional magnetohydrodynamic turbulence, J. F1uid Mech., 90 (1), 129.

Panchev, S. (1971). Random Function and Turbulence, Pergammon.

Patterson, G. S. and Orszag, S. A.(1971). Spectral calculations of isotropic turbulence: Efficient removal of aliasing interactions, Phys . Fluids, 14, 2538 .

Potter, D.(1972). Computationa1 Physics, John Wiley and Sons.

Robertson, H. P.(1940). The invariant theory of isotropic turbulence, Proc, Camb. Phi1. Soc., 36, 209.

Seyler, C. E., Salu, Y., Blontgomery, D. and Knorr, G.(1975). Two-dimensional turbulence in inviscid fluids or guiding center plasma, Phys. F1uids, $\underline{18}(7), 803$.

Shebalin, J. V.(1982). Anisotropy in MHD turbulence due to mean magnetic field, Ph.D. Thesis, College of William and Mary. 
Shebalin, J. V., Matthaeus, W. H, and Montgomery, D.(1983). Anisotropy in MHD turbulence due to mean magnetic fie1d, J. P1asma Phys.. 29 (3), 525 .

Taylor, G. I.(1935). Statistical theory of turbulence, Parts 1-4, Proc. Roy. Soc. A, 151, 421 .

Tay1or, G. I.(1938a). Production and dissipation of vorticity in a turbulent fluid, Proc. Roy. Soc. A, 164, 15.

Taylor, G. I.(1938b). The spectrum of turbulence, Proc. Roy. Soc. A, 164. 476 .

Townsend, A. A.(1947). The measurement of double and triple correlation derivatives in isotropic turbulence, Proc. Camb. Phil. Soc., 43, 560.

Tritton. D. J.(1977). Physical Fluid Dynamics, Van Nostrand Reinhold Company. 


\section{FIGURE $1 \mathrm{~A}$}

INITIAL MOOAL ENERCY SPECTRUM

FOR INVISCID NAVIER-STOKES RUN

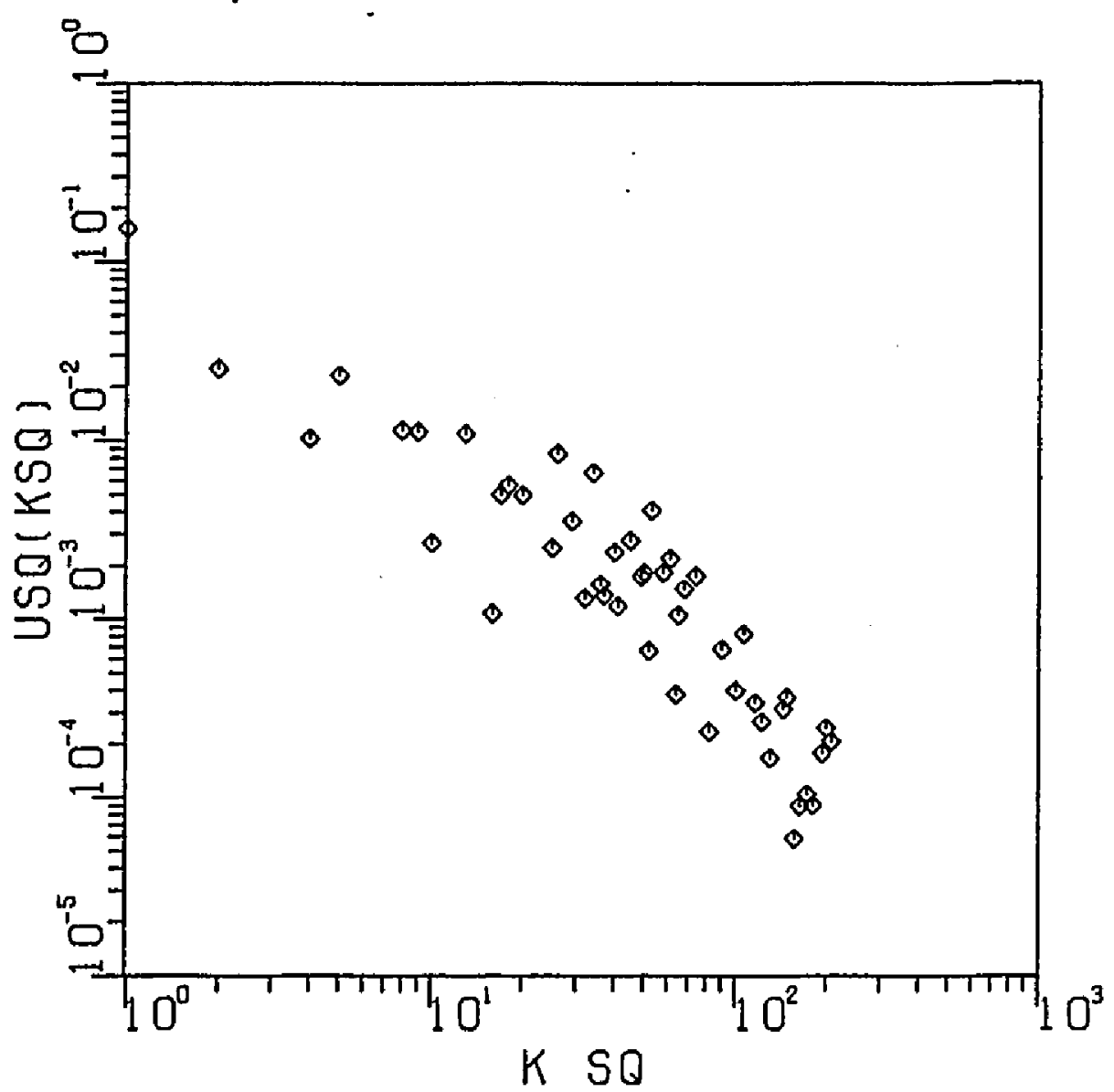


F IGURE $1 \mathrm{~B}$

MOOAL ENERGY SPECTRUM

INVISCIO NAVIER-STOKES RUN

AVERAGED OVER 1000 TIME STEPS ENDED AT $T=97.66$

SOLID CURVE IS THE EOUILIBRIUH PREOICTION

ALPHA $=-13.4$, BETA $=18.3$

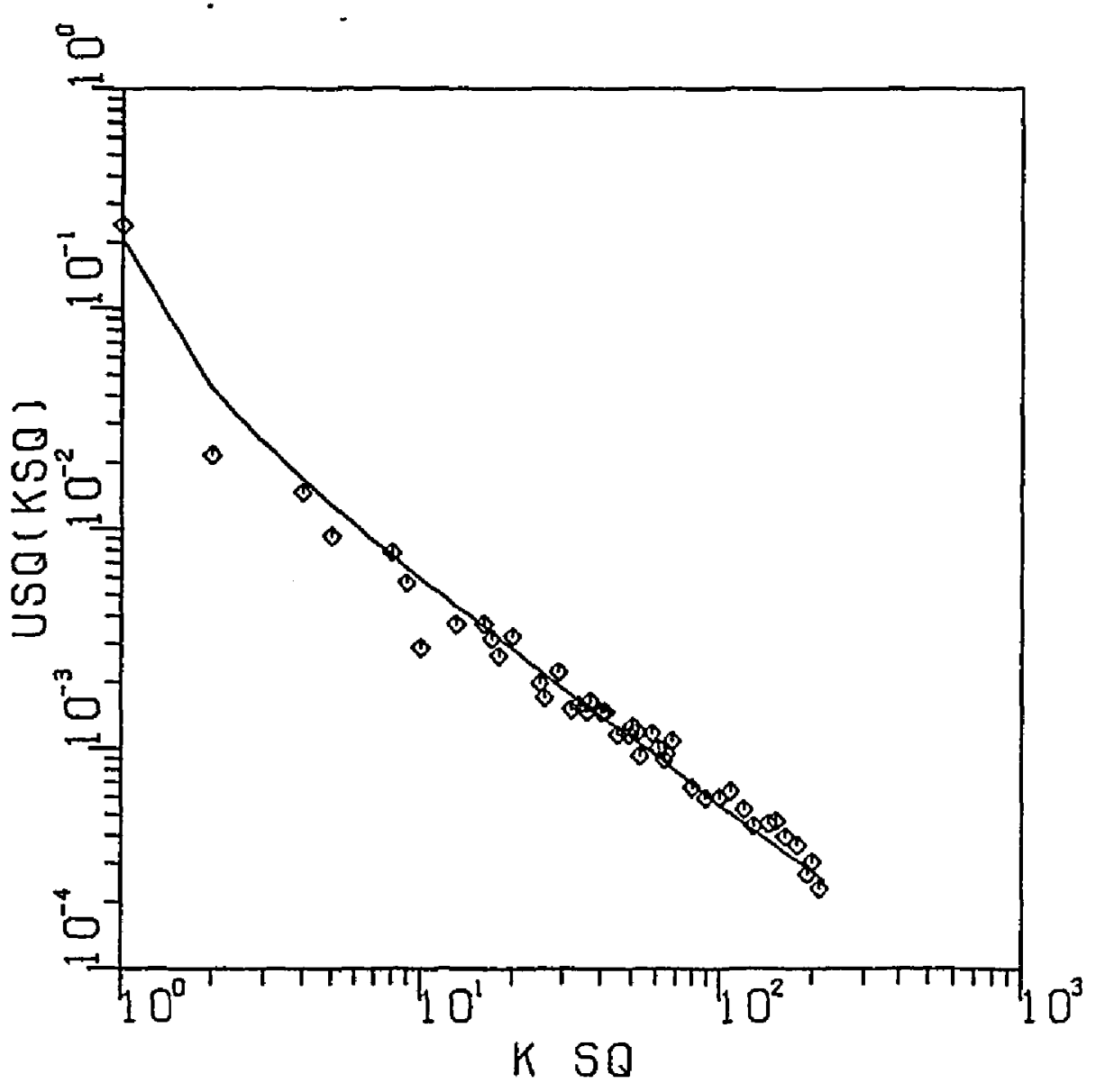


FIGURE $2 \mathrm{~A}$

INITIAL HECHANICAL MODAL ENERGY SPECTRUM

FOR NON-OISSIPATIVE HHD RUN

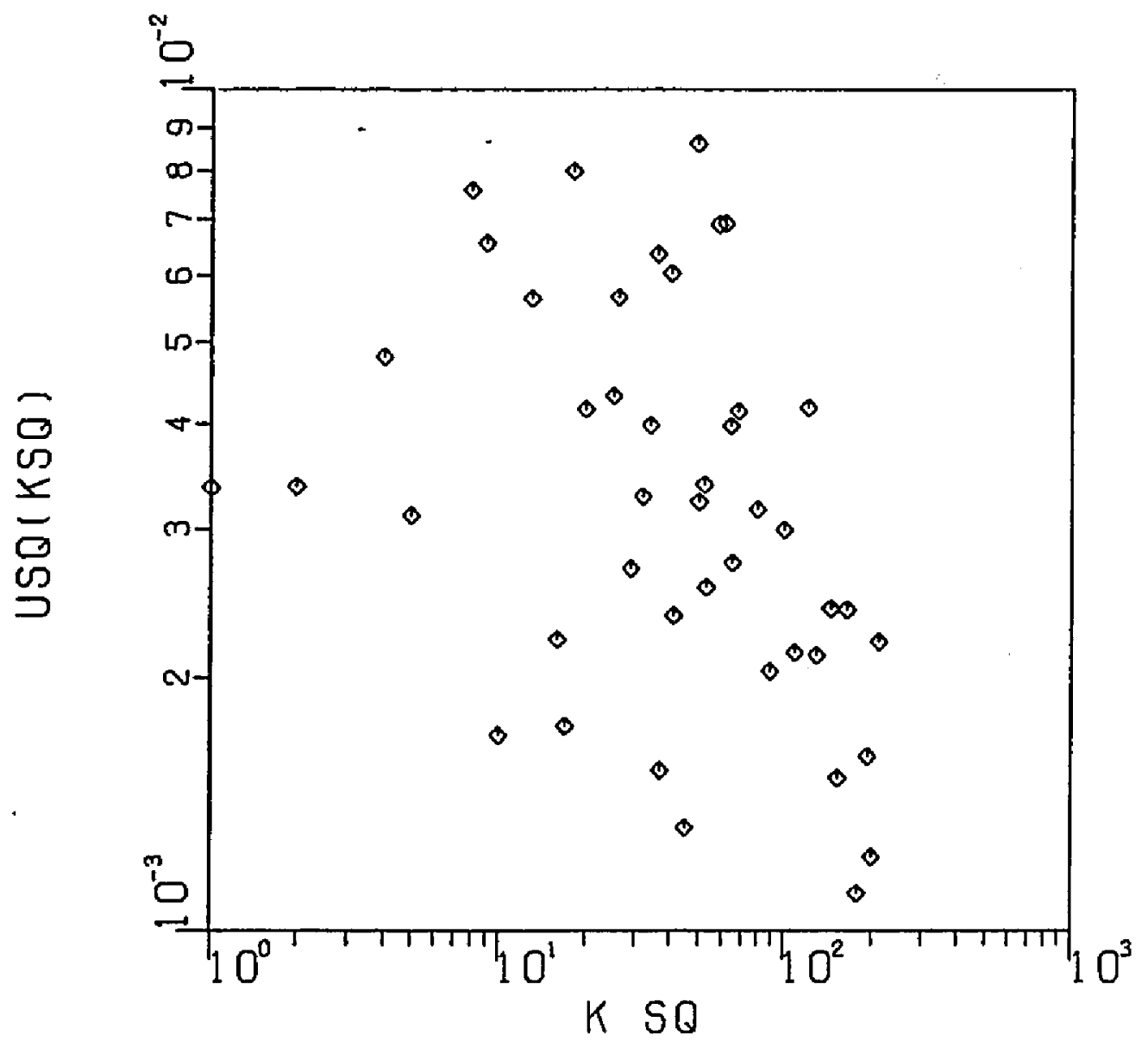




\section{FIGURE 2B}

INITIAL VECTOR POTENTIAL SPECTRUM

FOR NON-DISSIPRTIVE HHD RUN

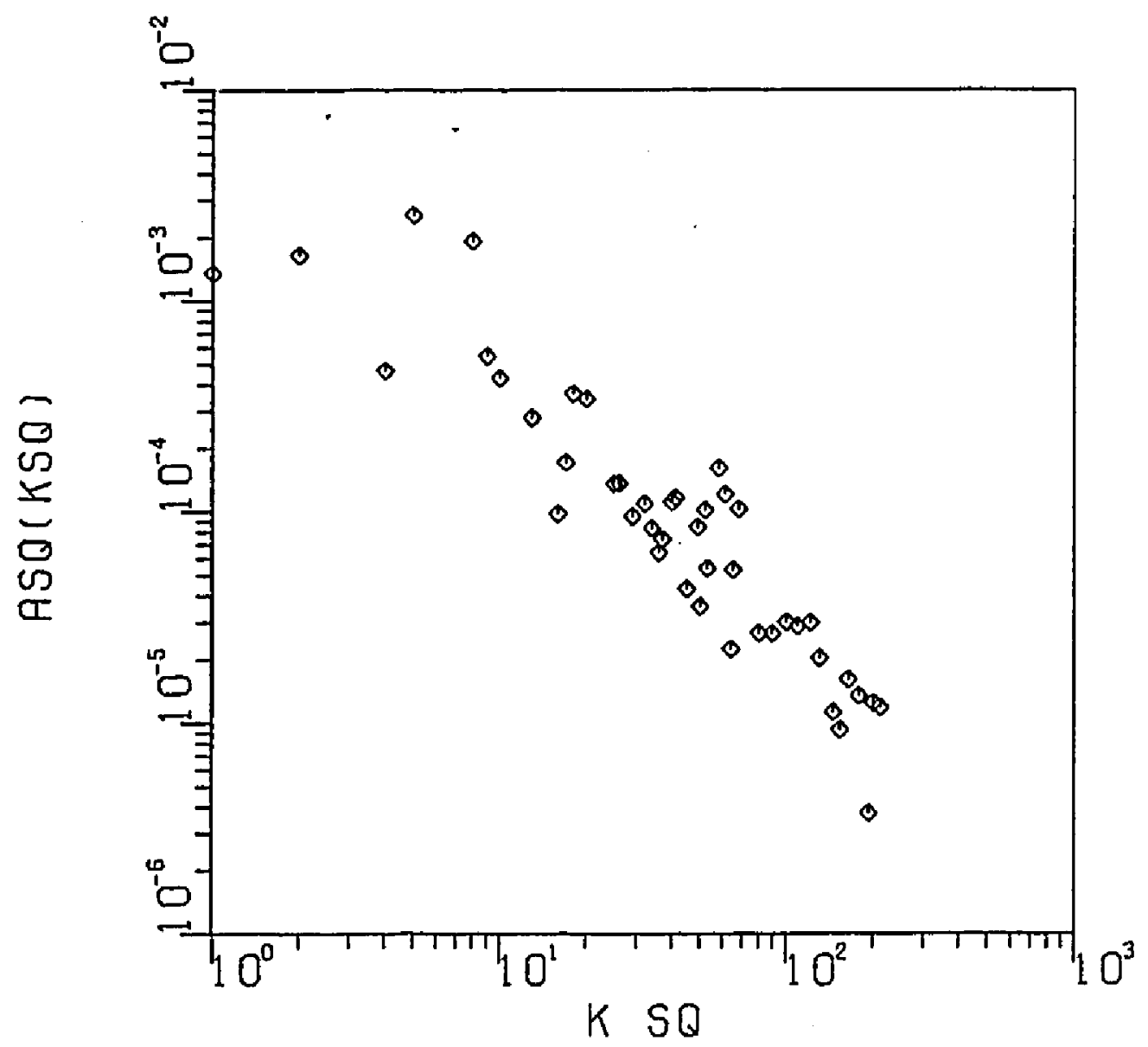




\section{F I GURE $\quad 2 \mathrm{C}$}

HECHANICAL MOOAL ENERGY SPECTRUM

NON-DISSIPATIVE HHD RUN

AVERAGED OVER 2000 TIHE STEPS ENDED AT $T=39.66$

SOLID CURVE IS THE EOUILIBRIUM PREOICTION

ALPHA $=714$, BETA $=0$, GAMMA $=-446$

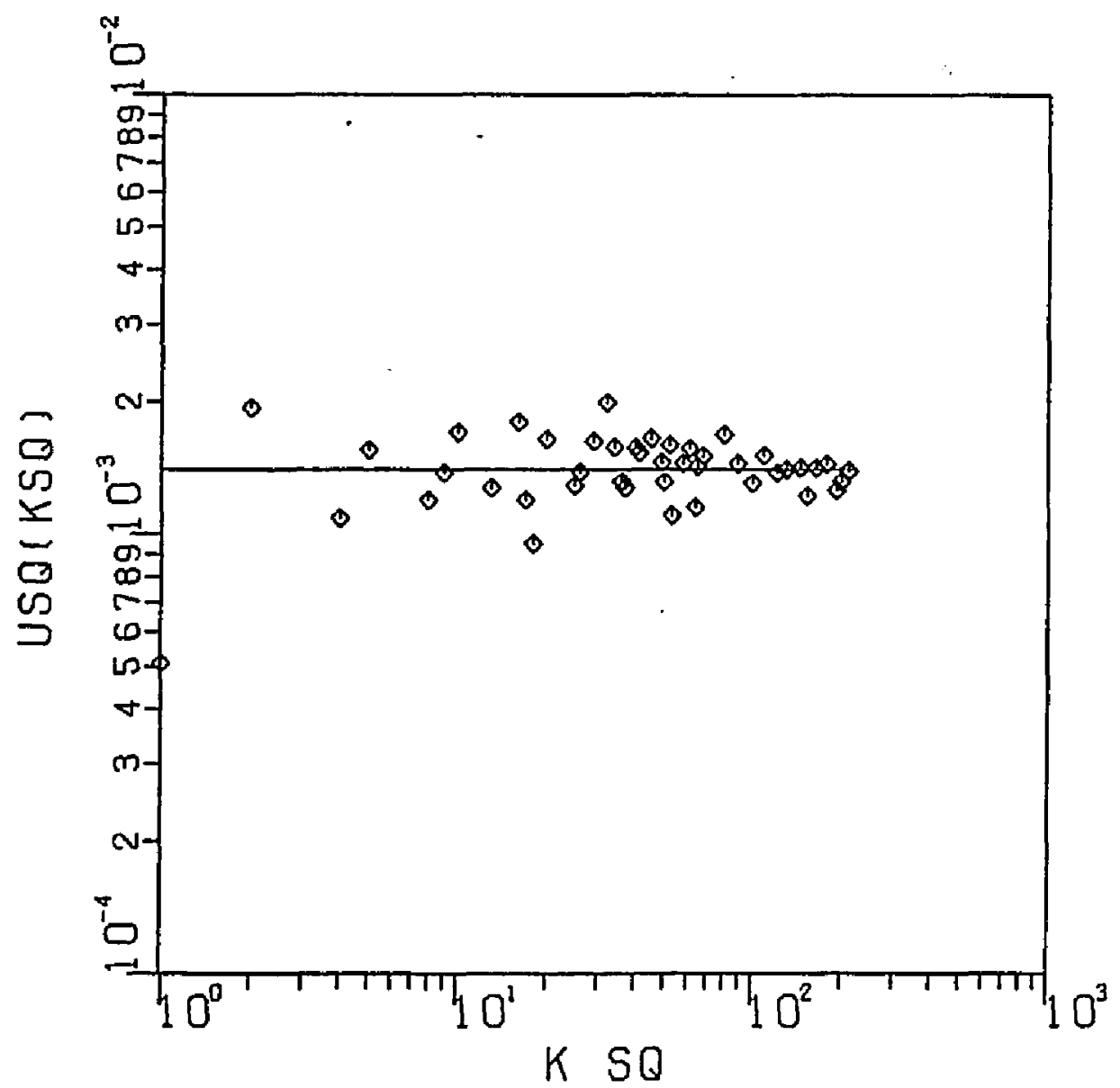




\section{F IGURE $2 D$}
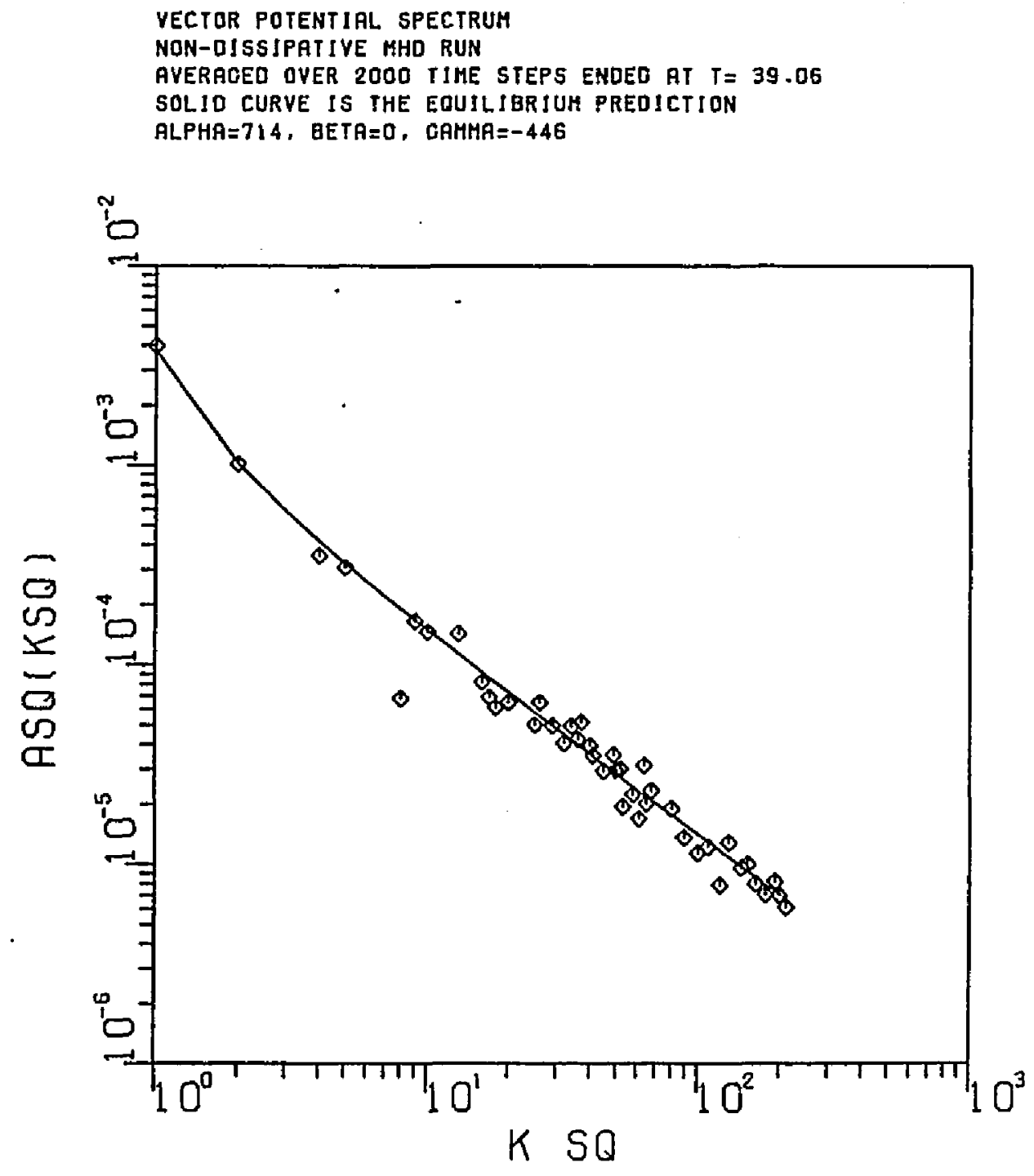
FIGURE $3 A$

ENERCY VERSUS TIHE

FOR DRIVEN NAVIER-STOKES RUN

ISOUARES REPRESENT THE CONTRIBUTION

DUE TO THE FUNDAMENTAL, )

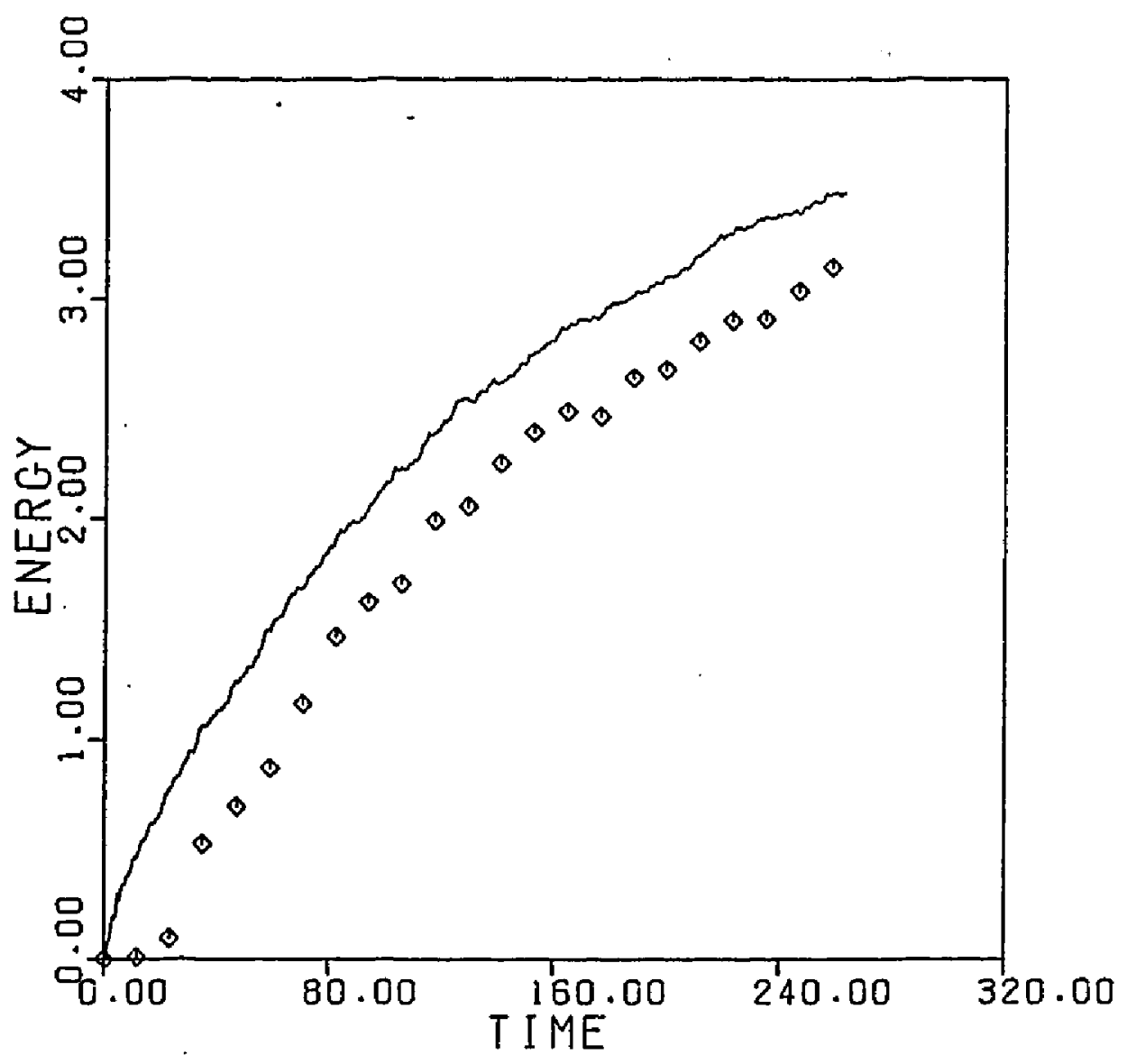




\section{FIGURE 3B}

ENSTROPHY VERSUS TIME

FOR ORIVEN NAYIER-STOKES RUN

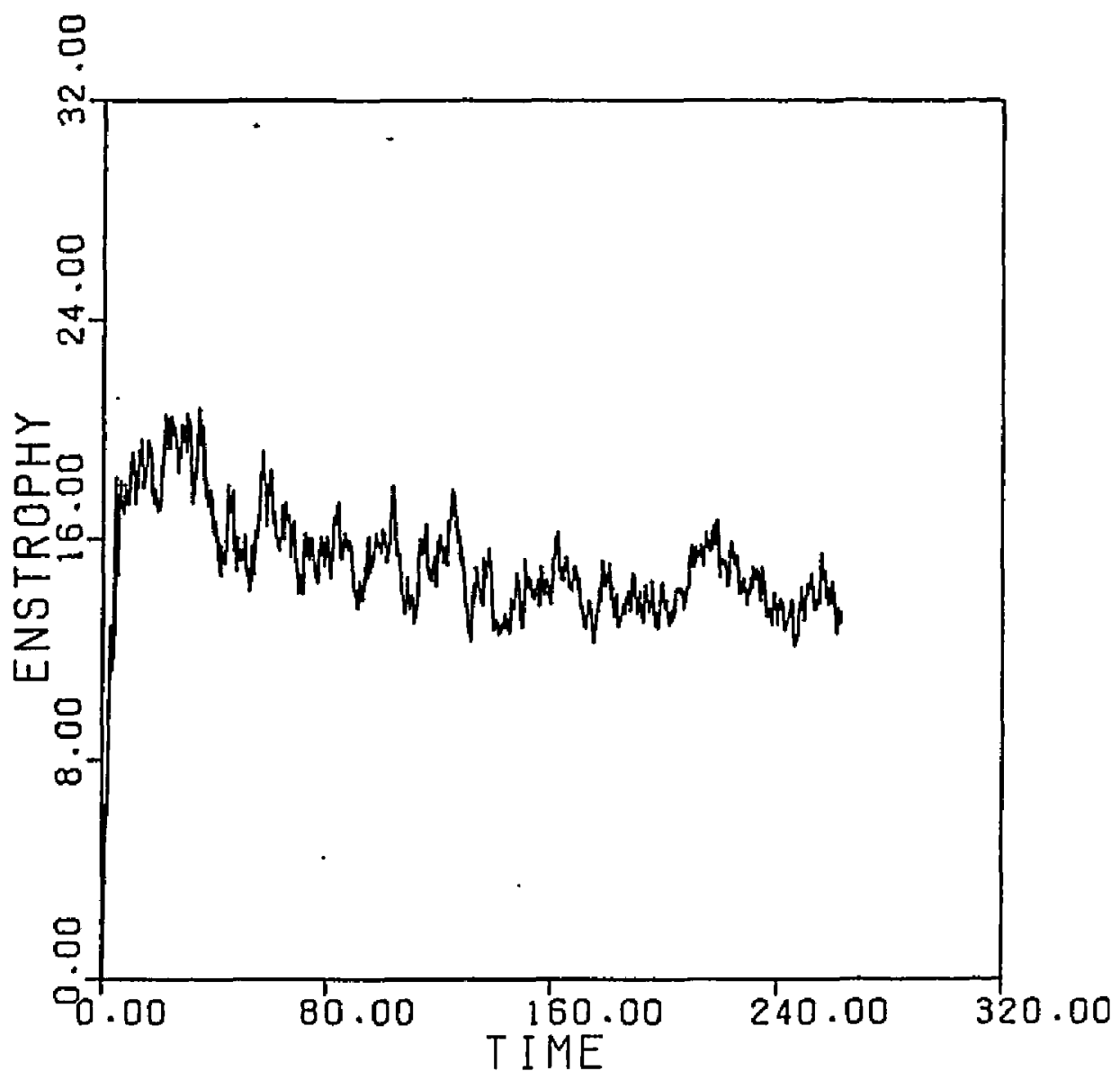


FIGURE $\quad 4 \mathrm{~A}$

MODAL ENERGY SPECTRUH

AVERACED OVER SOD TIHE STEPS ENDED AT $T=19.53$

DRIVEN NAYIER-STOKES RUN

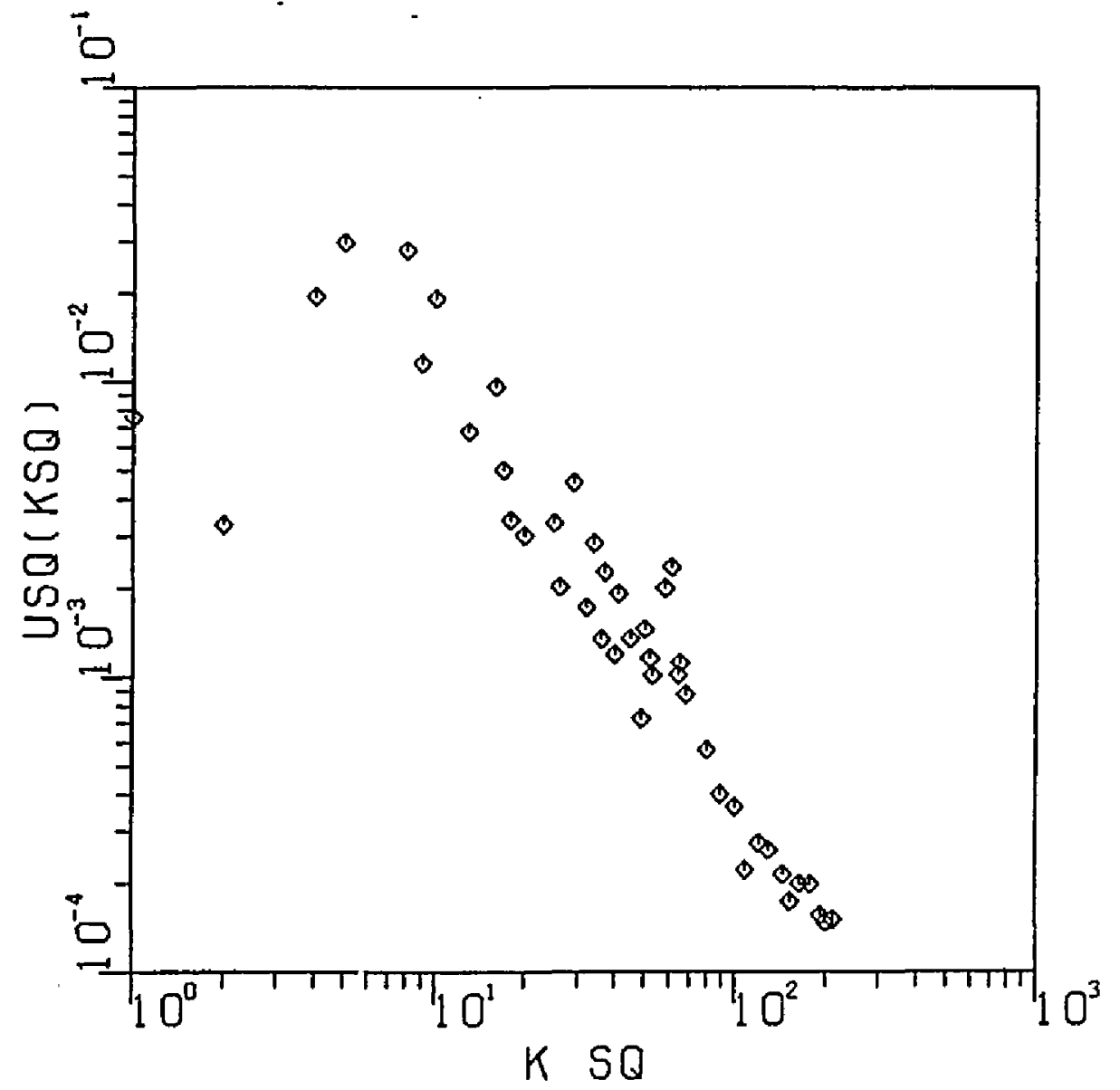




\section{FIGURE 4B}

MOOAL ENERGY SPECTRUM

FYERAGED OVER 1000 TIME STEPS ENDED AT $T=29.30$

DRIVEN NAVIER-STOKES RUN

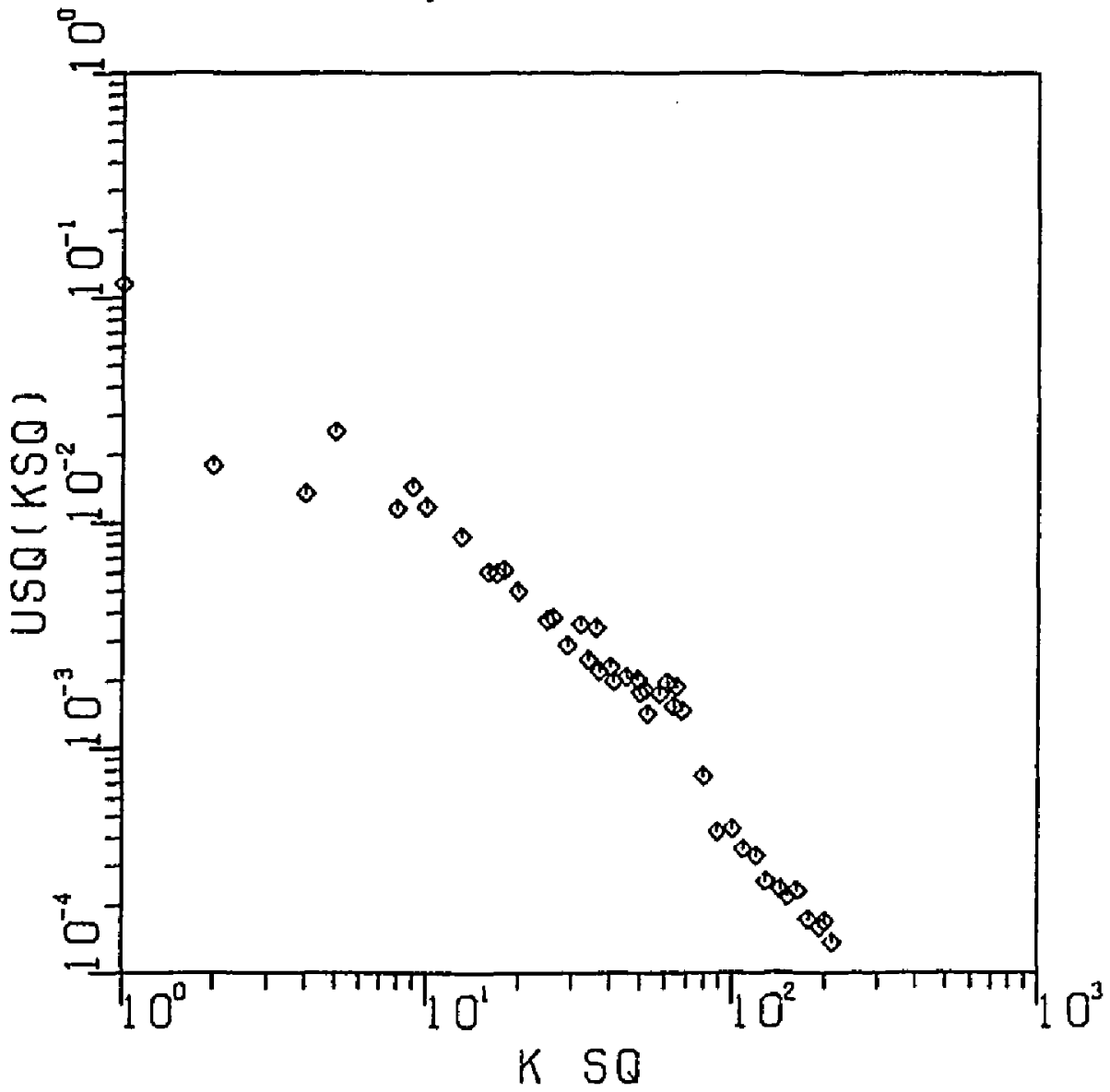


FIGURE $\quad 4 C$

MODAL ENERGY SPECTRUM

AVERACED OVER 1 DOO TIME STEPS ENOED AT $T=273.44$

DRIVEN NAVIER-STOKES RUN

SOLID LINES ARE

POHER LAH LEAST SOUARE FIT

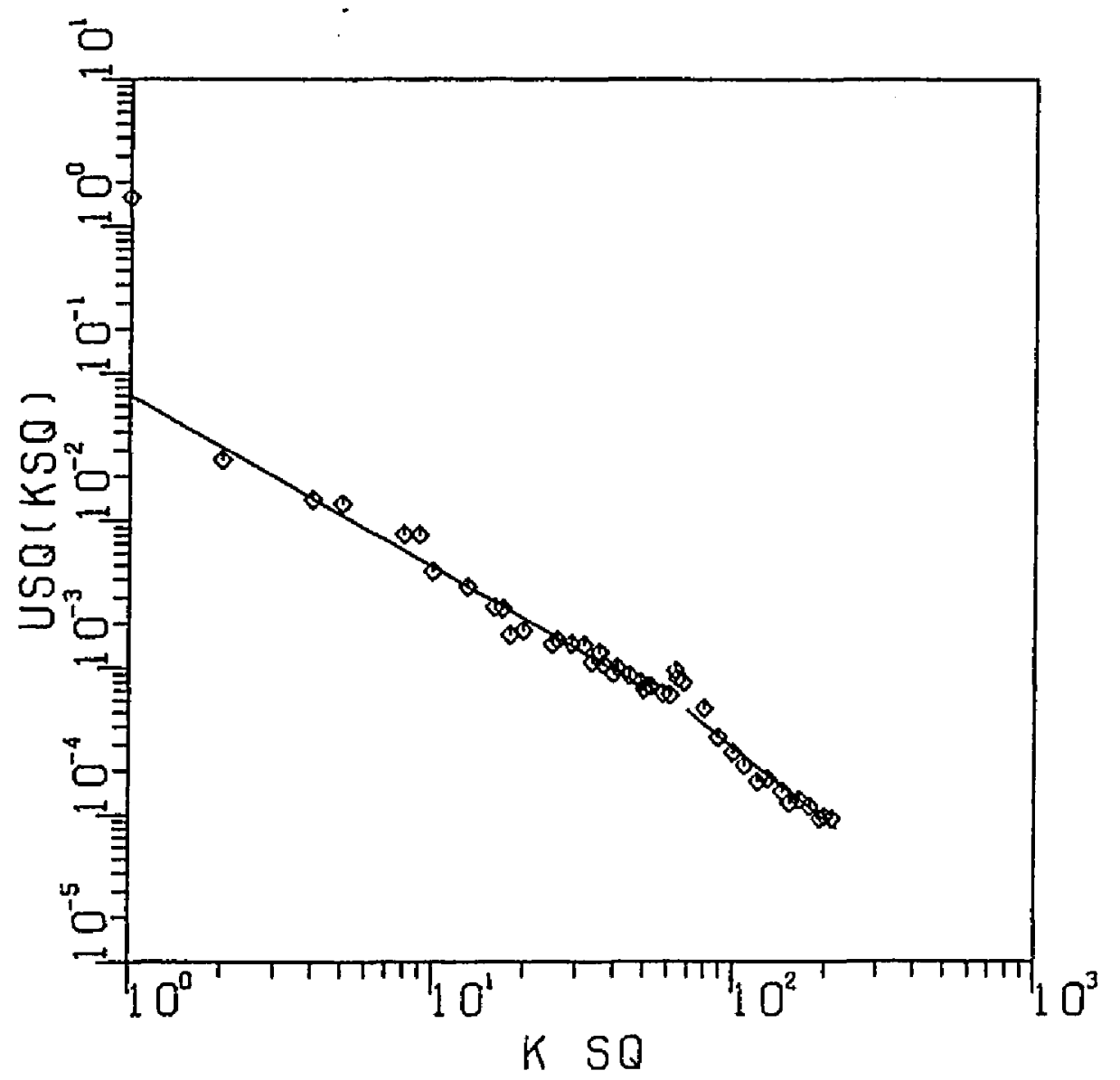




\section{FIGURE $5 \mathrm{~A}$}

CONTOURS OF STREAM FUNCTICN

AT $T=19.53$. TIME STEP 5000

FORCED NAVIER-STOKES RUN

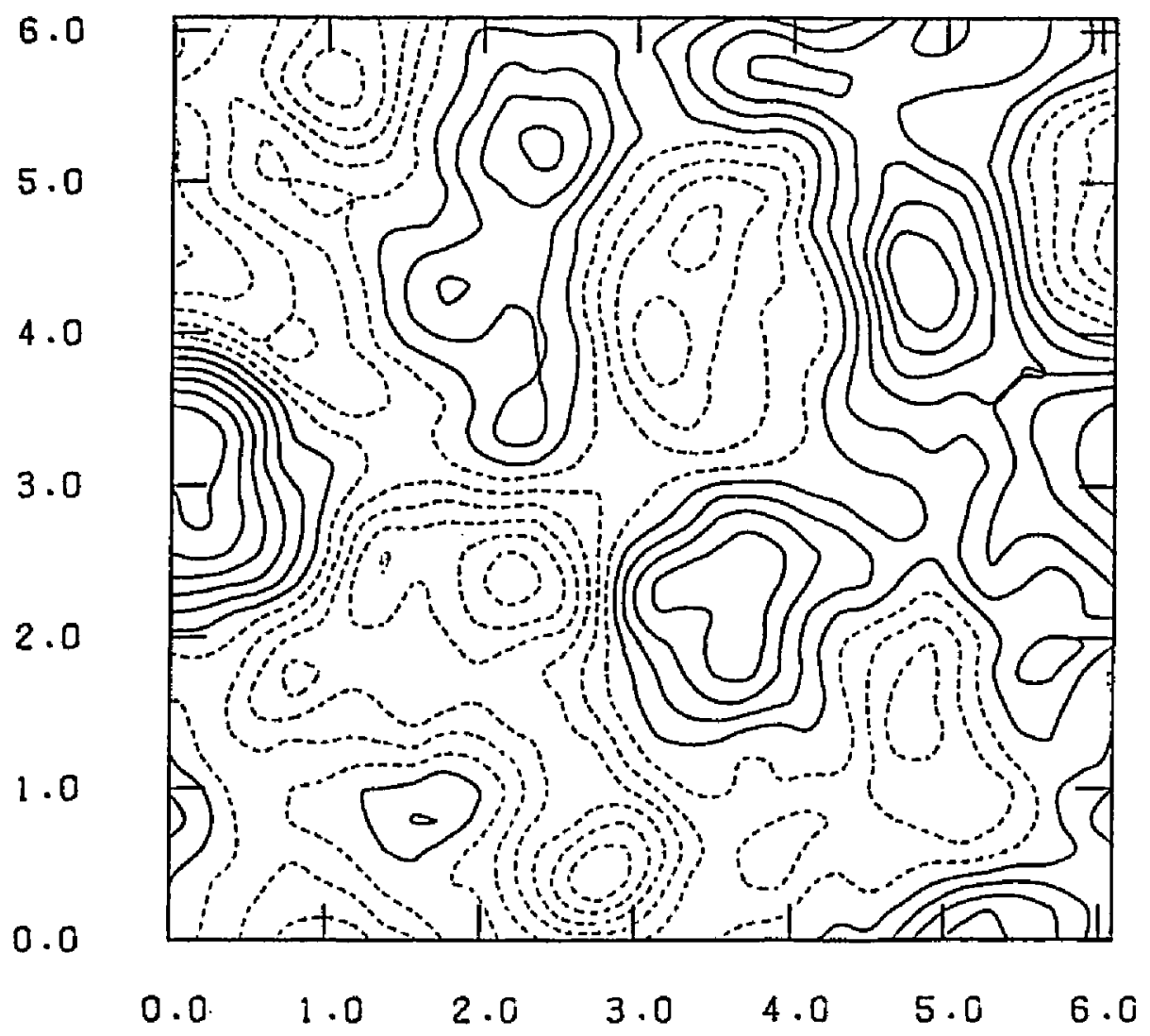




\section{F IGURE 5B}

C.ONTOURS OF STREAH FUNCTION

AT $T=97.66$. TIHE STEP 2500D

FORCED NAVIER-STOKES RUN

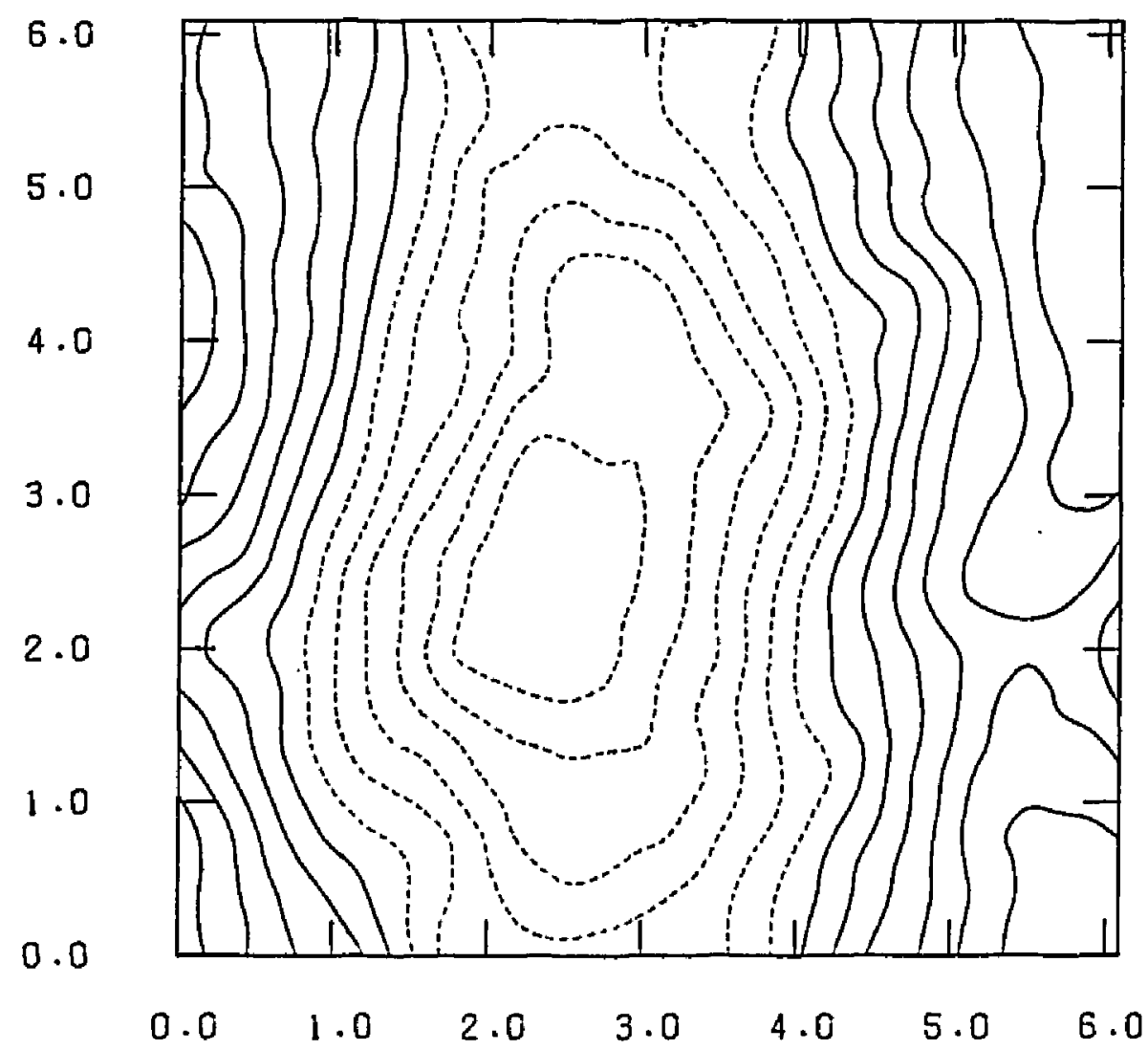


FIGURE $5 C$

CONTOURS OF STREAM FUNCTION

AT $T=273.44$. TIME STEP 70000

FORCED NAVIER-STOKES RUN

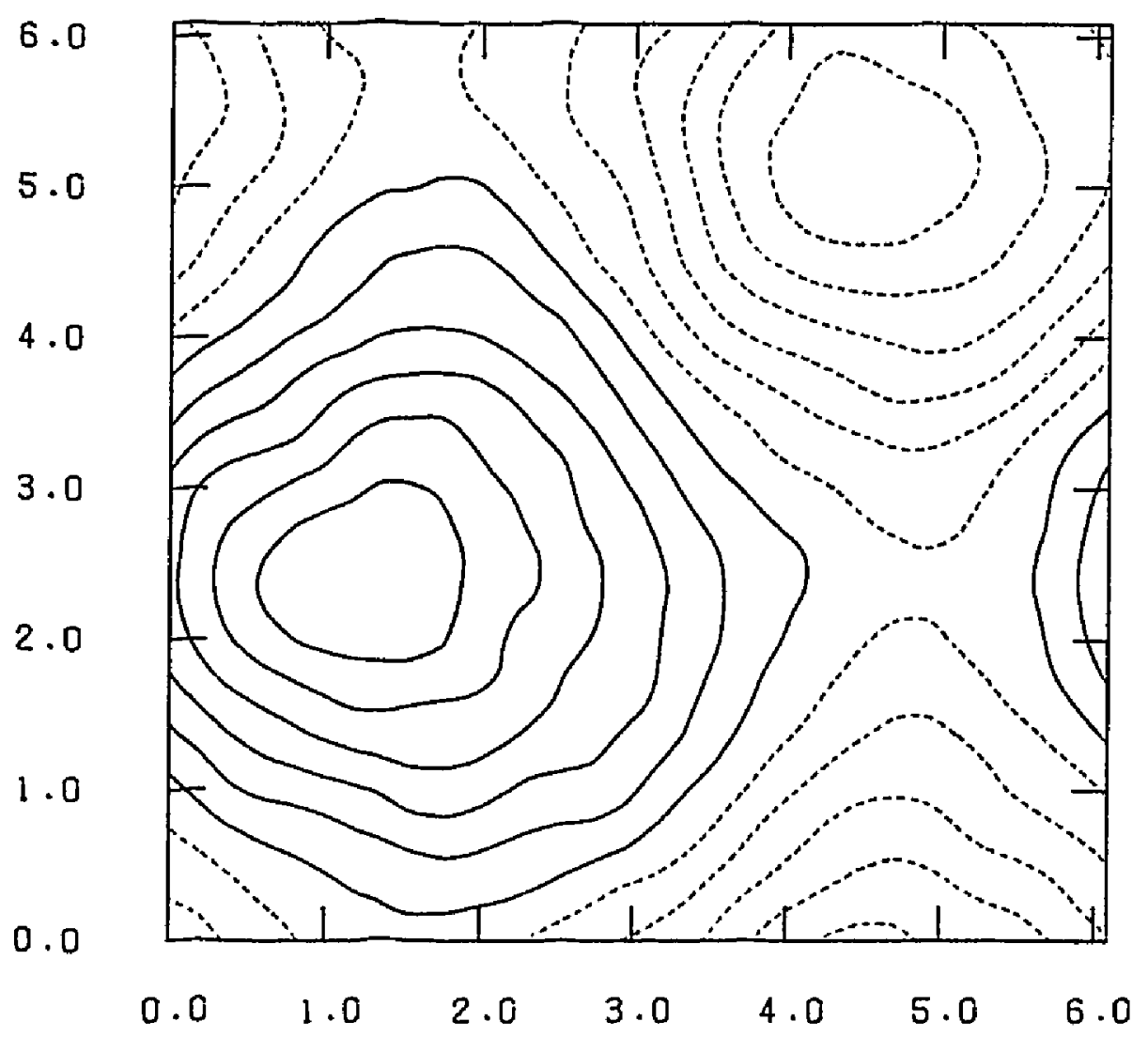




\section{FIGURE 5D}

CONTOURS OF VORTICITY DENSITY

AT $T=19.53$, TIME STEP 5000

FORCED NAVIER-STOKES RUN

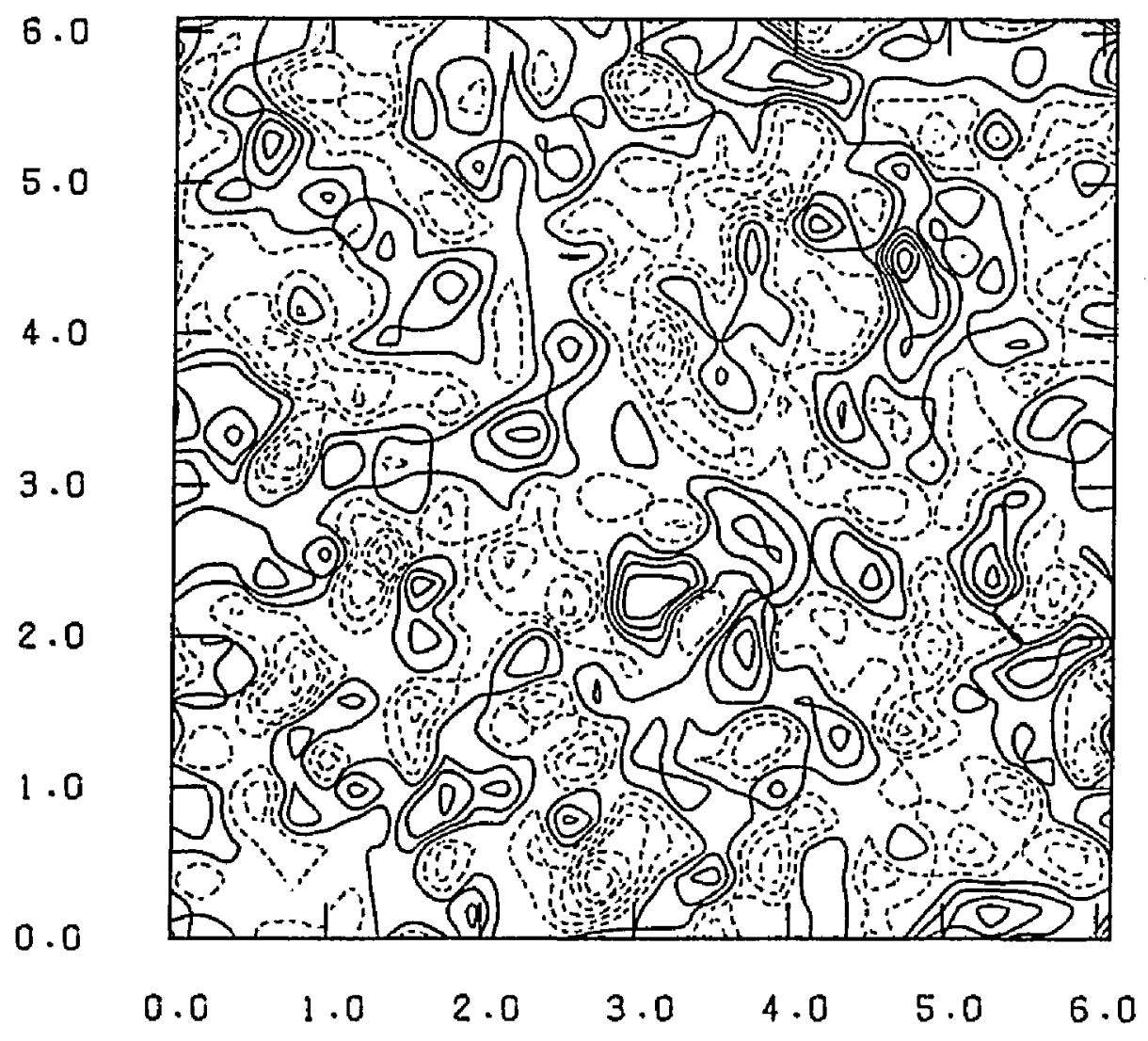




\section{FIGURE 5E}

CONTOURS OF YORTICITY DENSITY

AT $T=97.66$. TIME STEP 25000

FORCED NAVIER-STOKES RUN

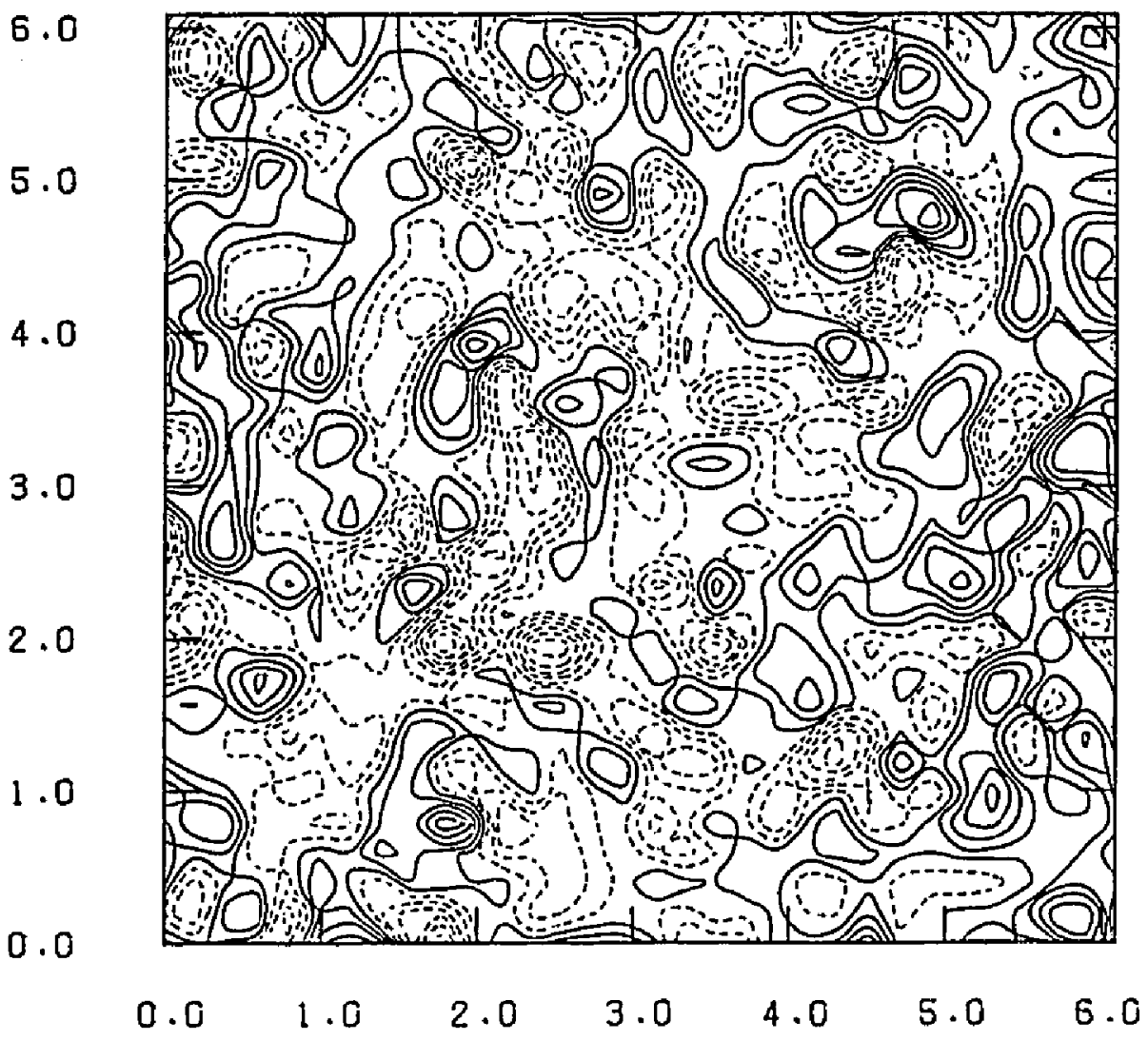




\section{FIGURE $5 F$}

CONTOURS OF VORTICITY DENSITY

AI $T=273.44$, TIME STEP 70000

FORCED NAYIER-STOKES RUN

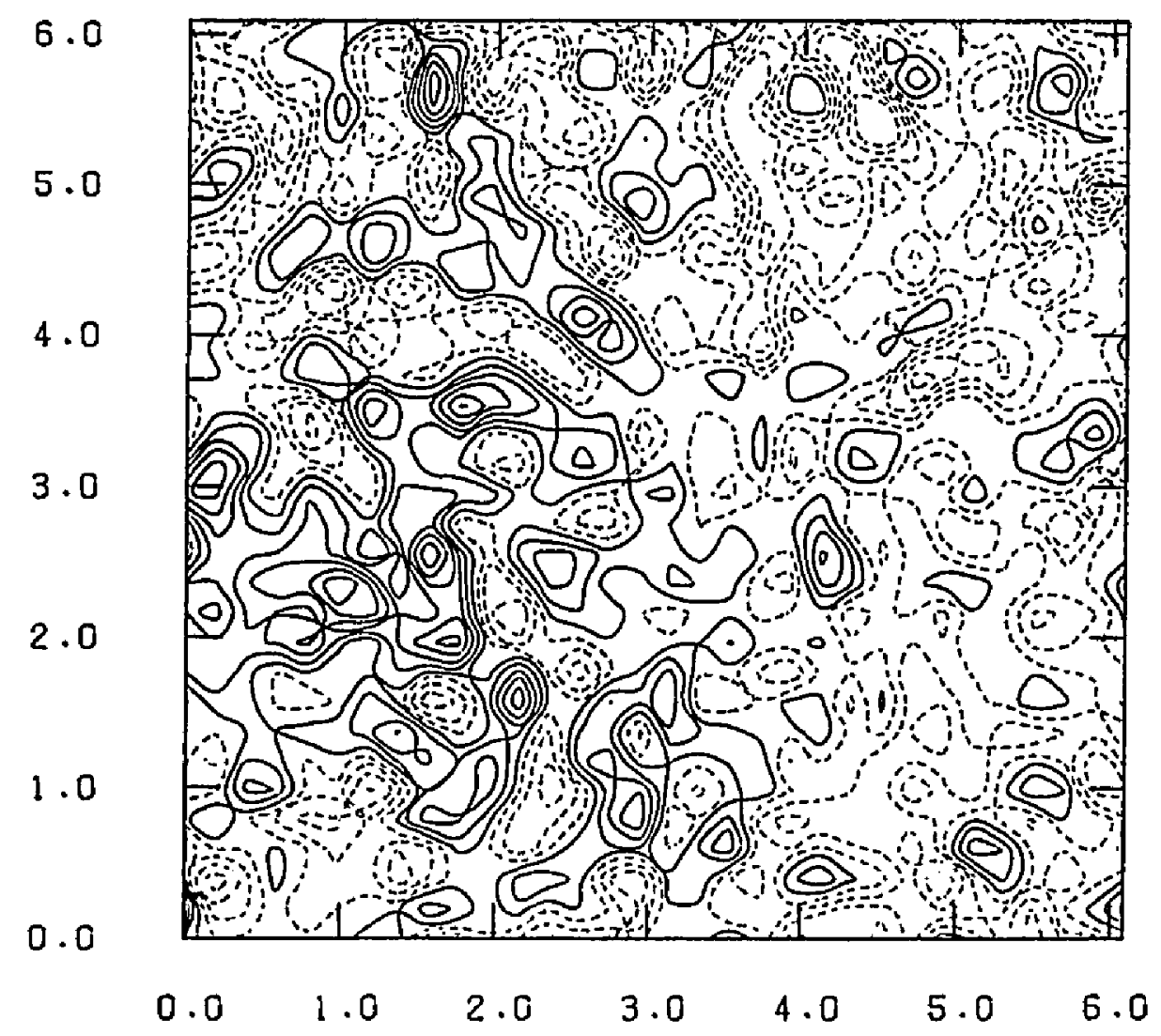


FIGURE 6 A
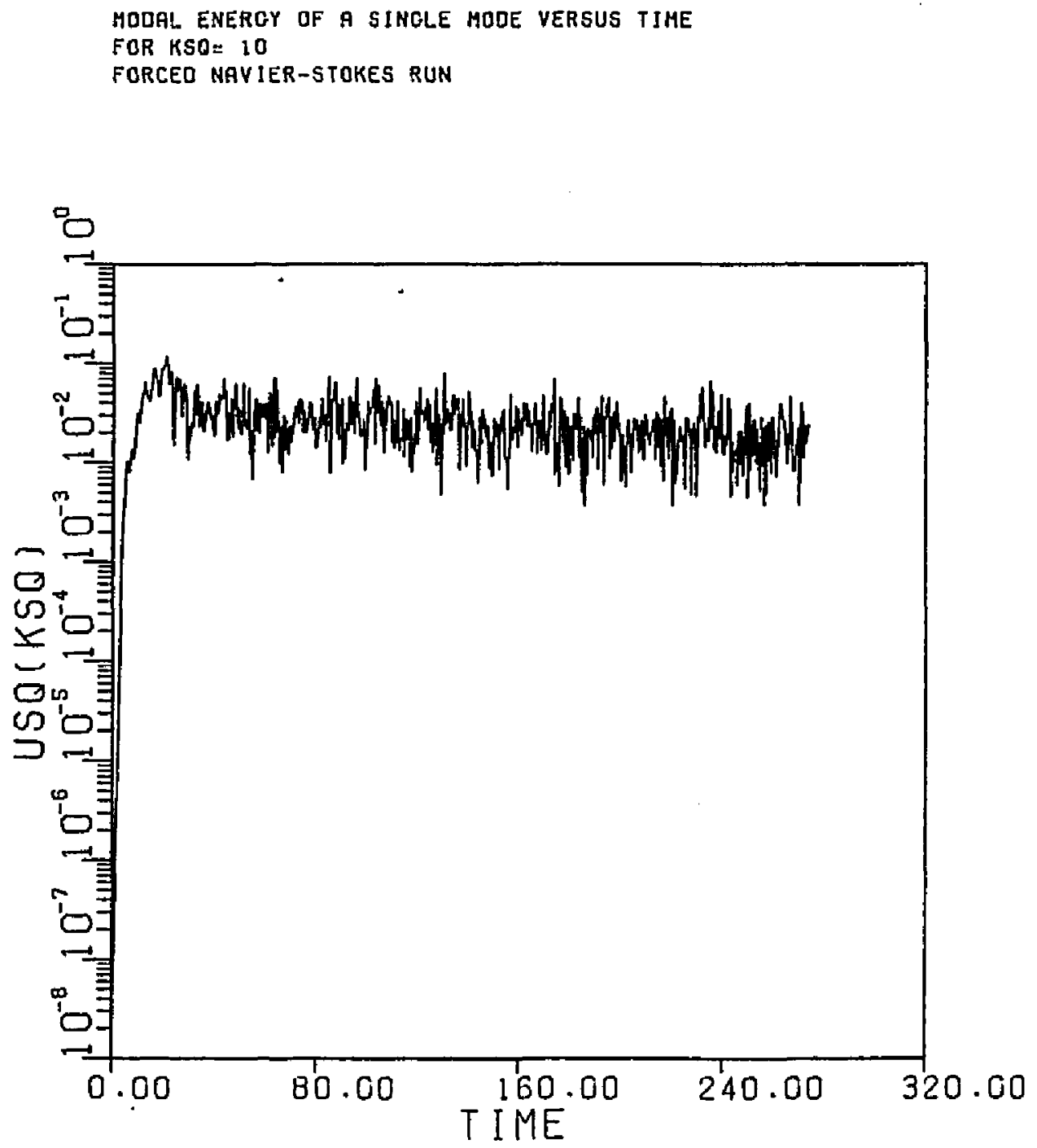


\section{F I GURE GB}

MODAL. ENERTY OF a SINOLE MODE VERSUS TIME FOR $K S O=25$

FORCED NAVIER-STOKES RUN

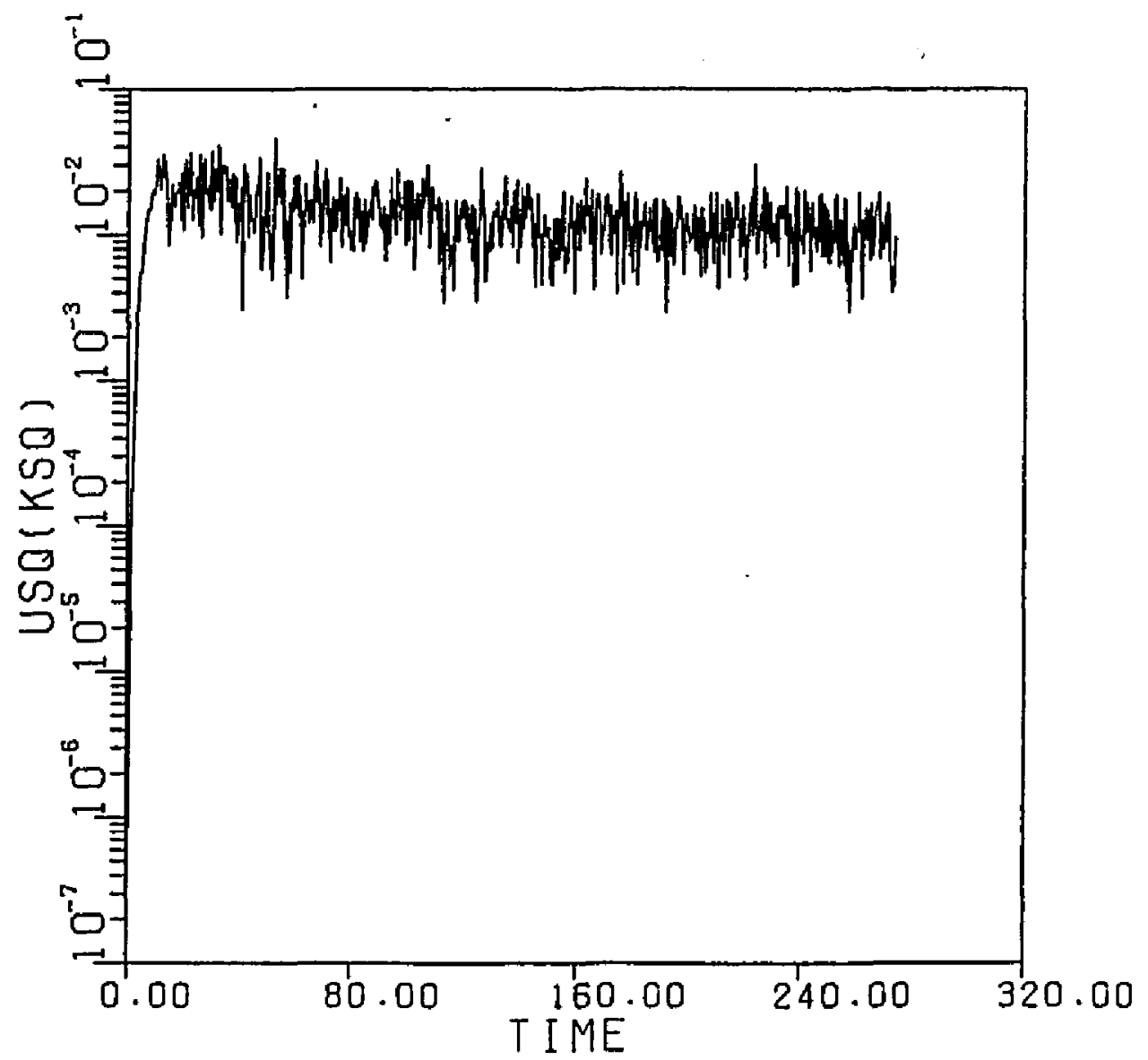


FIGURE GC

MODAL ENERGY OF A SINGLE MODE VERSUS TIME

FOR $K S O=40$

FORCED NAVIER-STOKES RUN

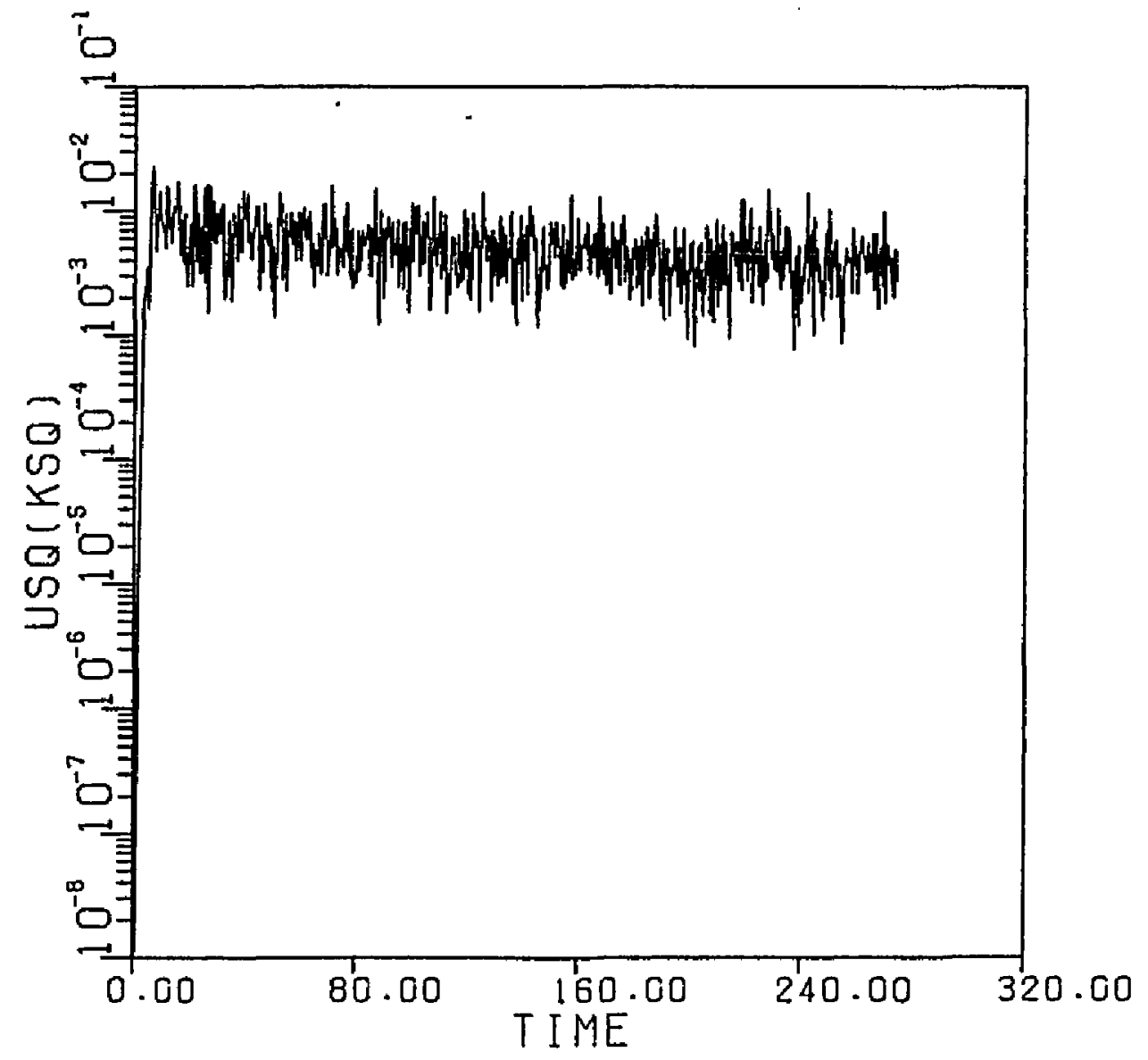


FIGURE $\quad 6 D$

MODAL ENERGY OF F SINGLE MODE VERSUS TIME

FOR KSQ = 65

FORCED NAVIER-STOKES RUN

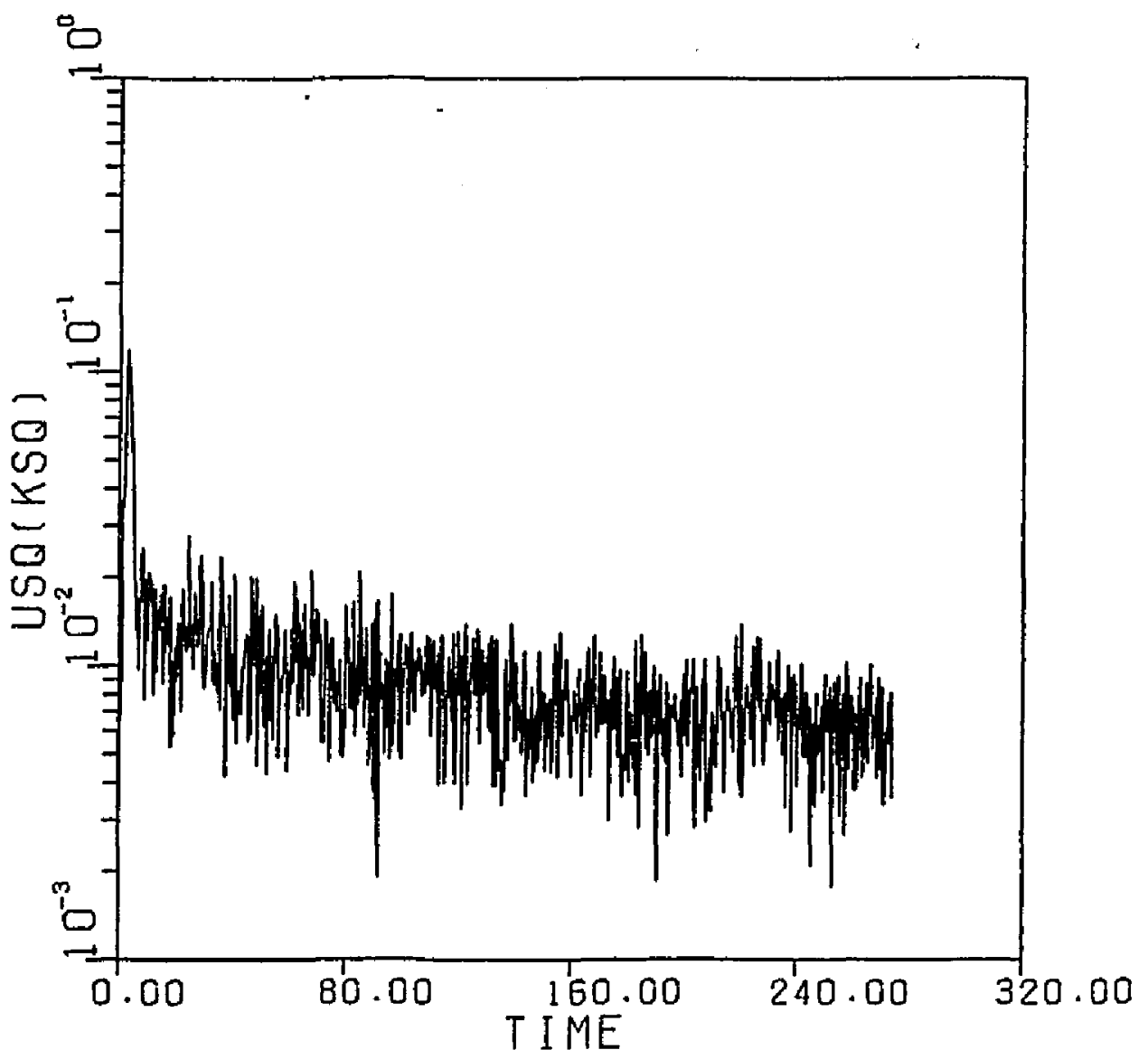


FIGURE GE

MODAL ENERGY OF A SINGLE MODE VERSUS TIME

FOR $K S O=85$

FORCED NAYIER-STOKES RUN

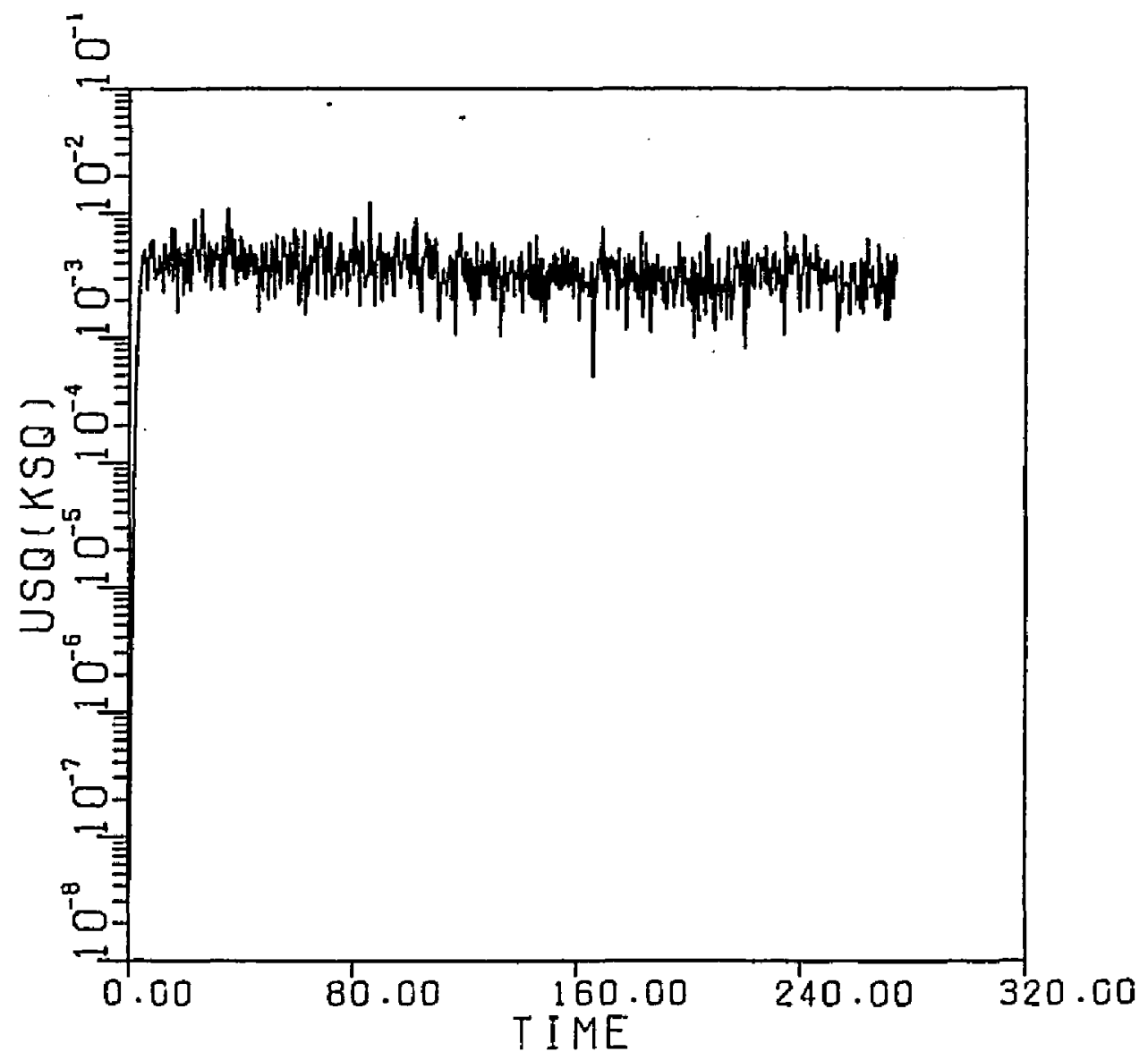




\section{FIGURE GF}

MODAL ENERGY OF A SINGLE MODE VERSUS TIME FOR KSO $=109$

FORCED NAVIER-STOKES RUN

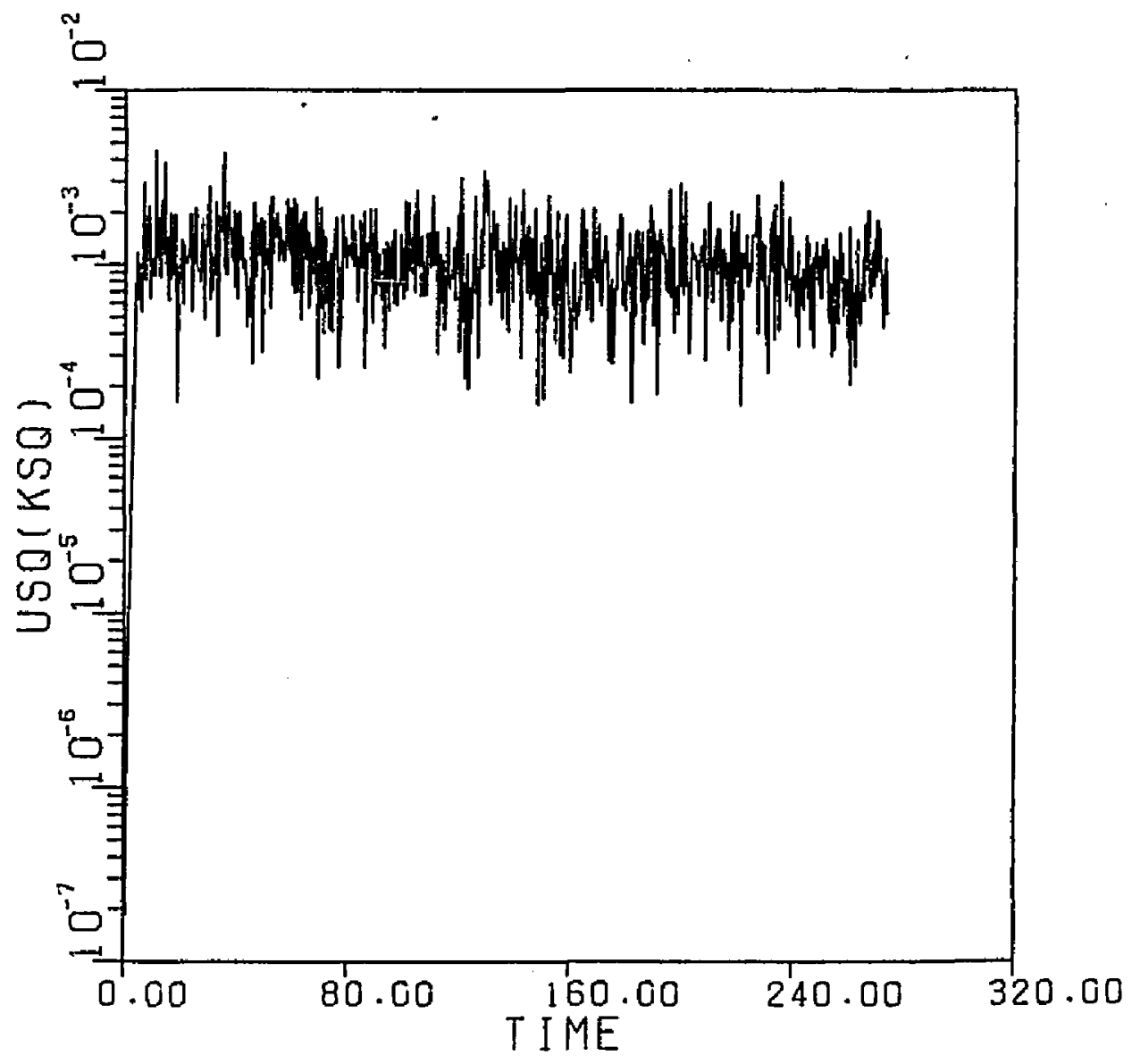




\section{FIGURE $\quad$ GG}
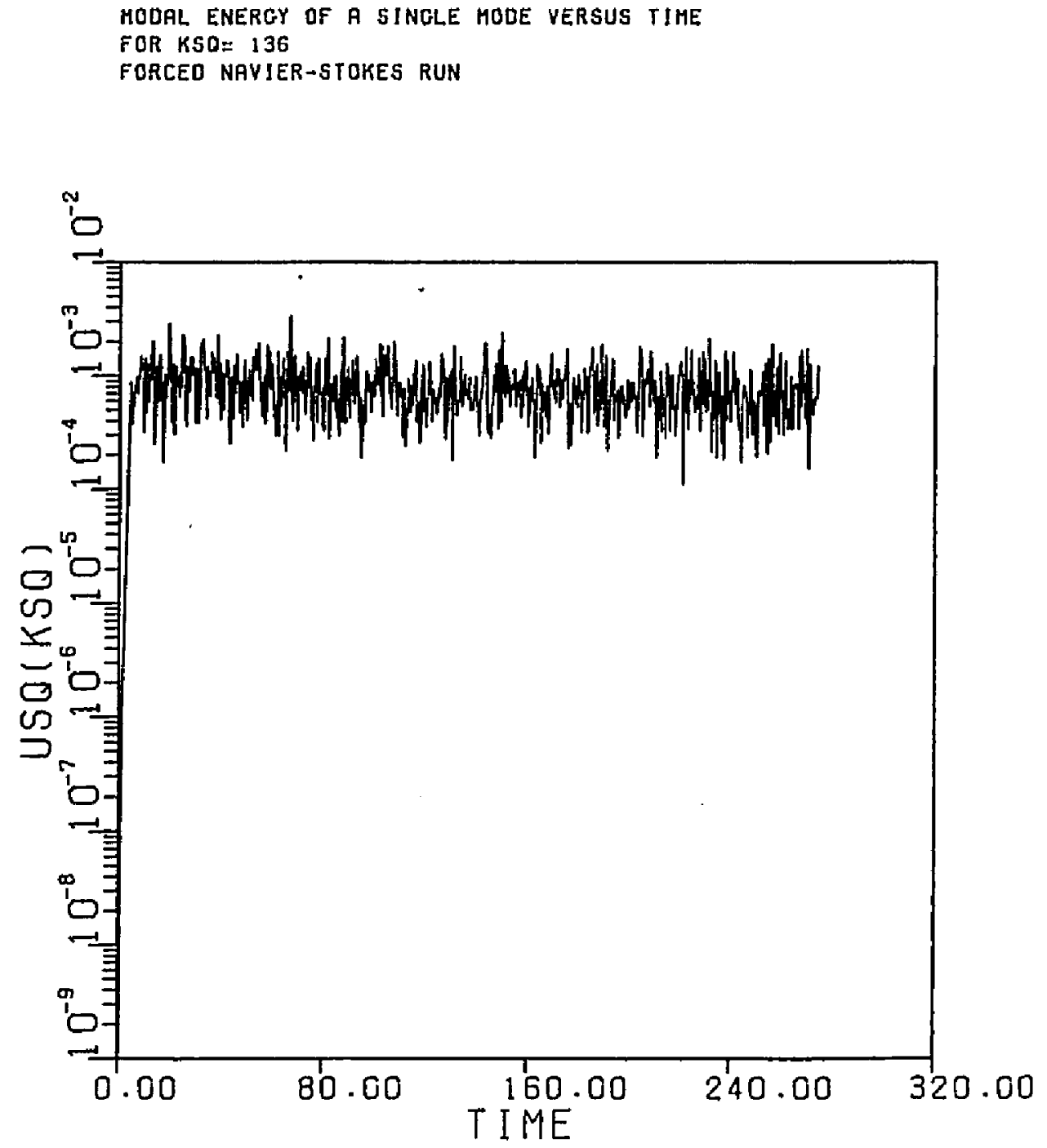
FIGURE $\quad$ GH

HODAL ENERGY OF A SINGLE HOOE VERSUS TIME

FOR KSO $=153$

FORCED NAVIER-STOKES RUN

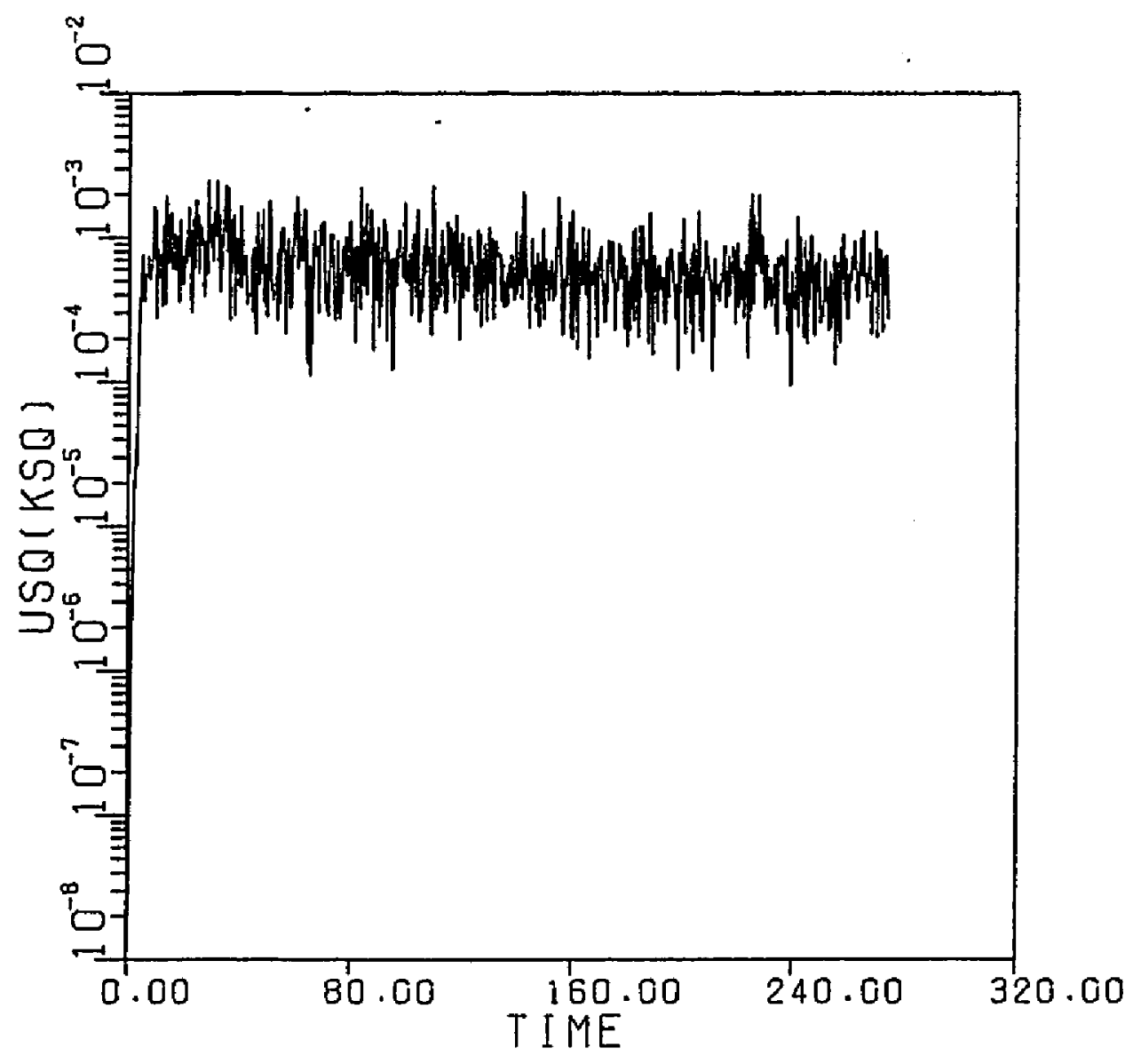


FIGURE 6 I

MODAL. ENERGY OF A SINGLE MODE VERSUS TIME

FOR KSG $=185$

FORCED NAVIER-STOKES RUN

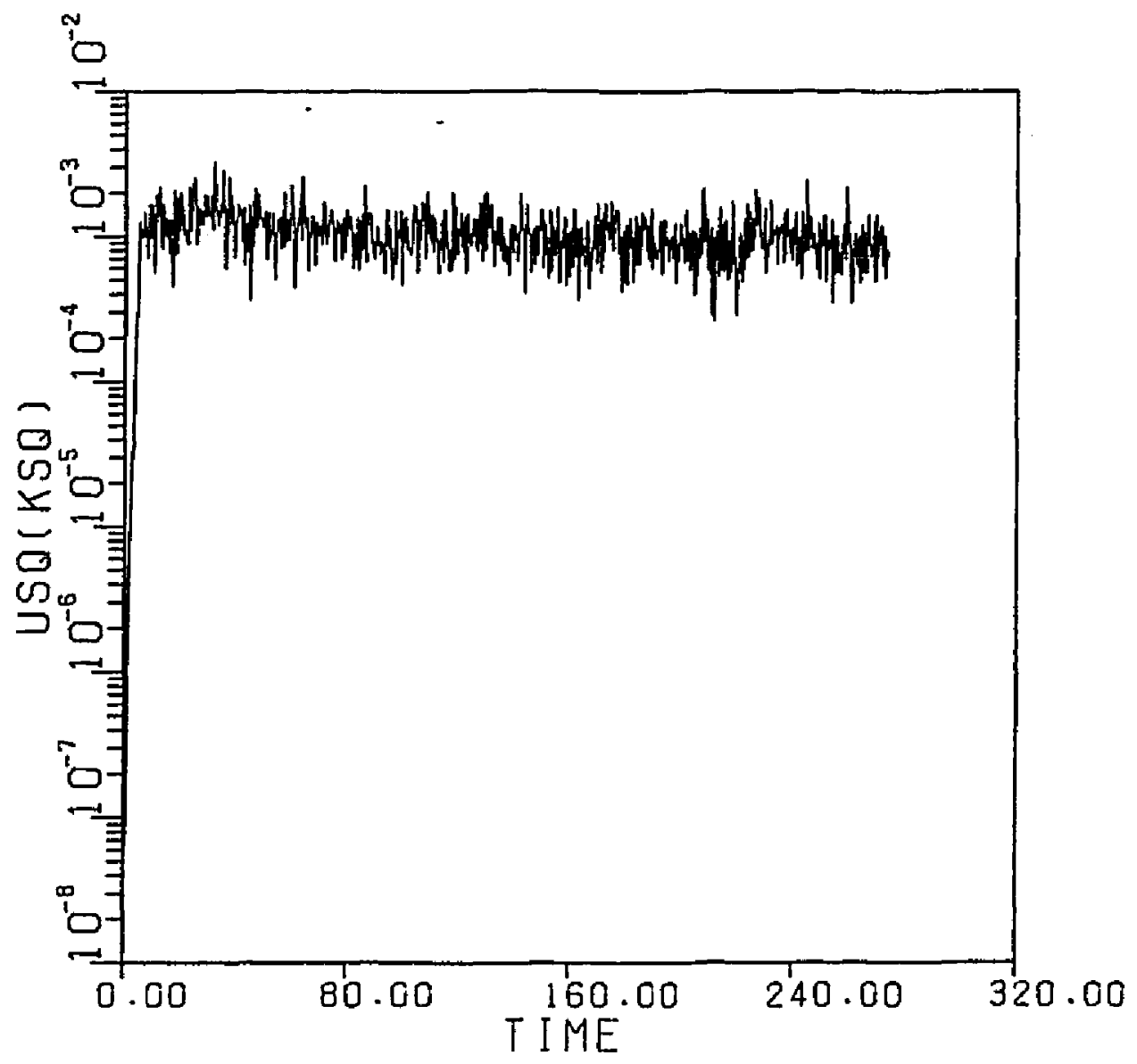


FIGURE GJ

MODAL ENERGY OF A SINGLE MODE VERSUS TIME $F O R$ KSO $=218$

FORCEO NAVIER-STOKES RUN

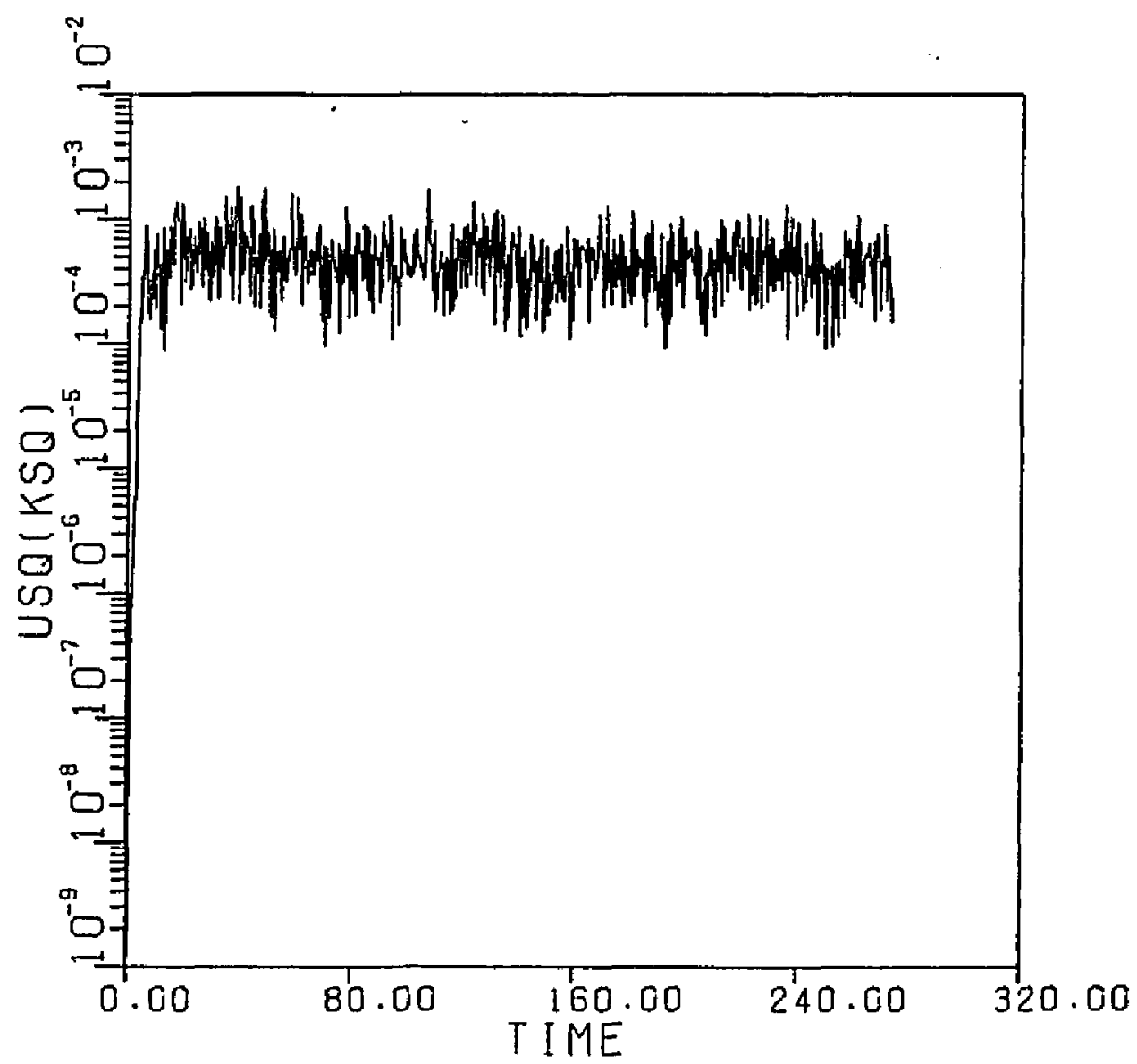


FIGURE 7

CARNEYALE ENTROPY VERSUS TJME

FOR DRIVEN NAVIER-STOKES RUN

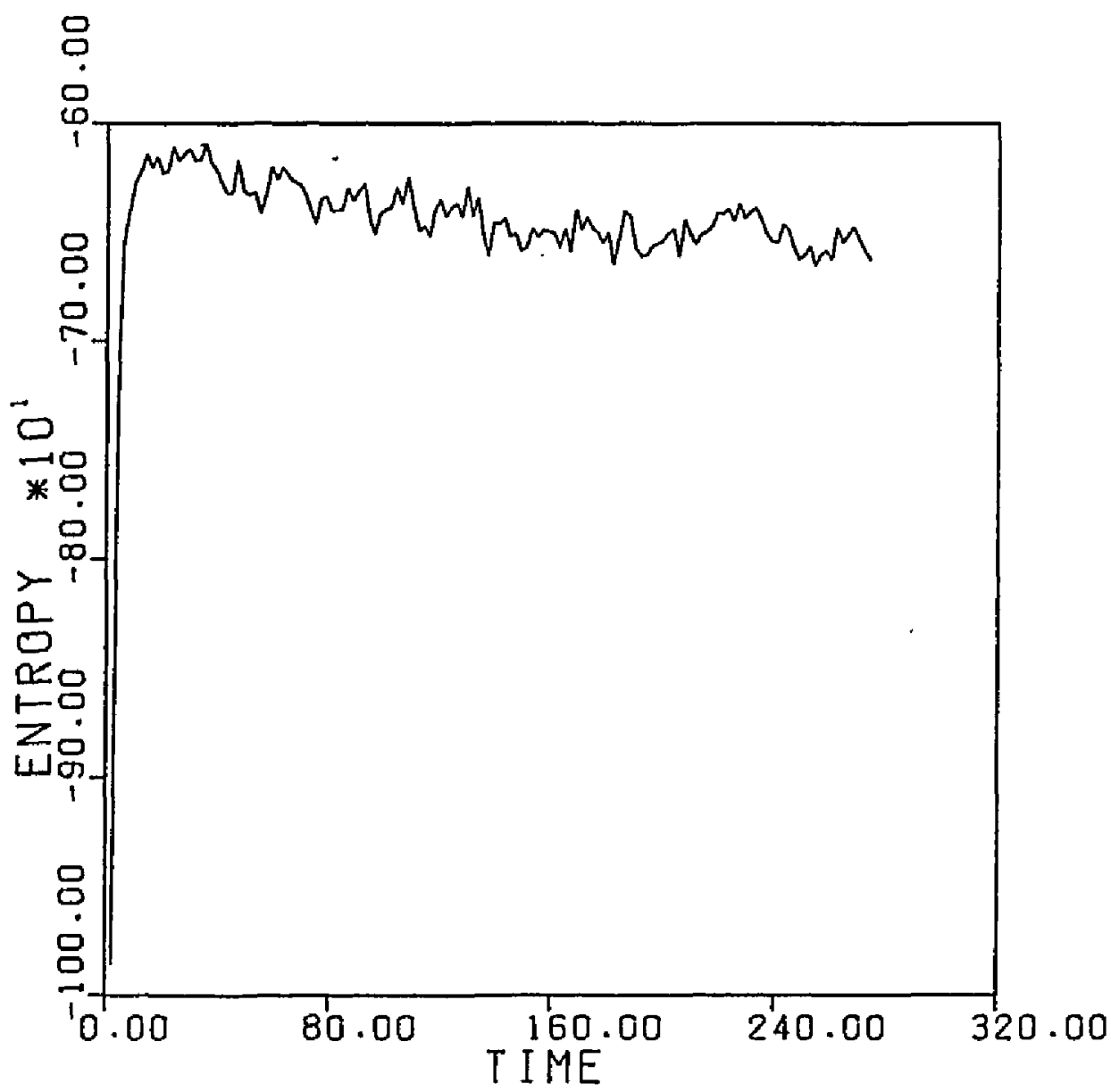


FIGURE $8 \mathrm{~A}$

ENERGY YERSUS TIME

DRIVEN MHD RUN

MECHANICAL CONTRIBUTION LABELED AS $V$

MACNETIC CONTRIBUTION LABELED AS B

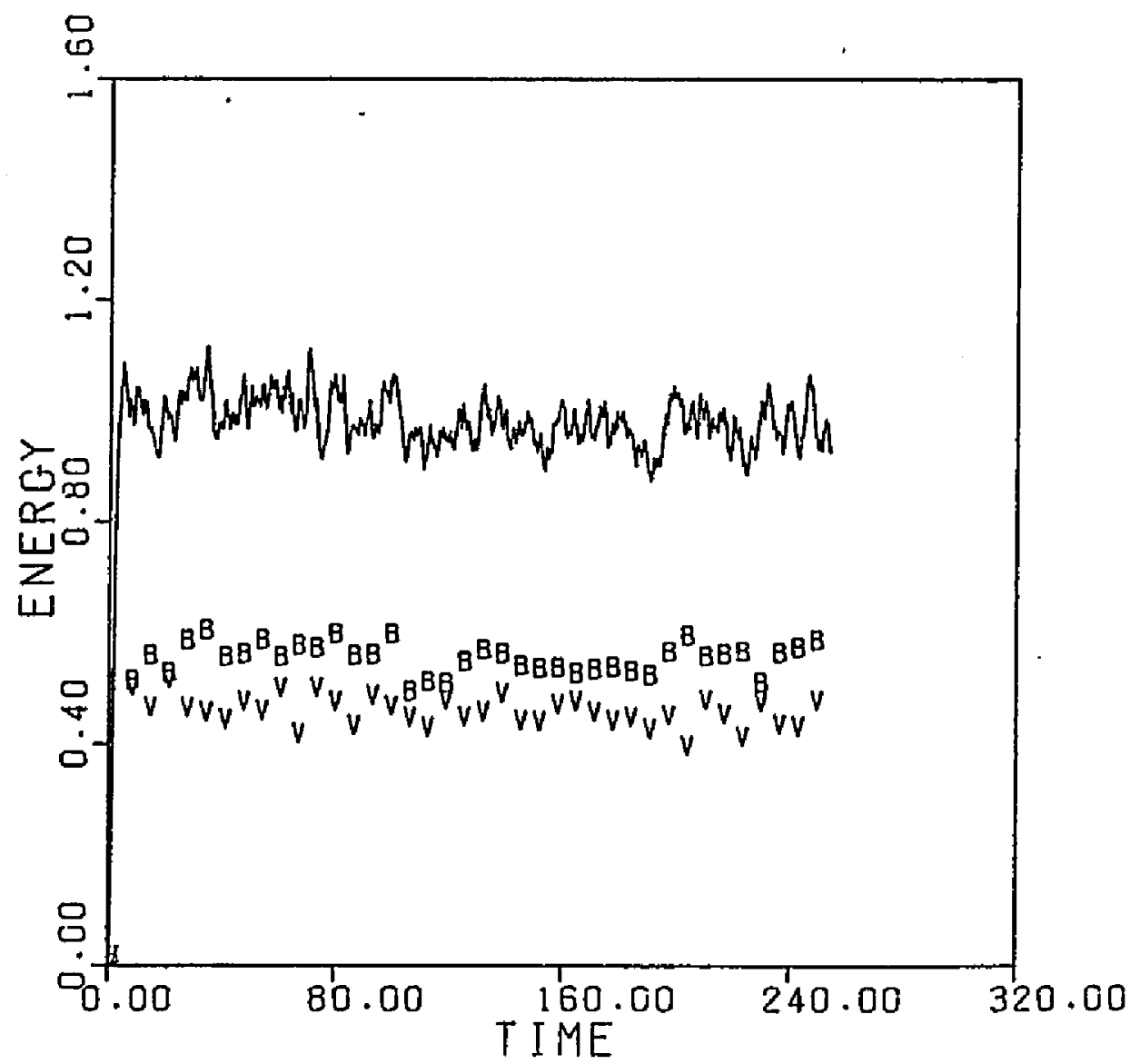




\section{FIGURE 8B}

MEAN SOUARE VECTOR POTENTIAL VERSUS TIHE DRIVEN MHO RUN

SOUARES REPRESENT THE CONTRIBUTION DUE TO THE FUNDAHENTAL

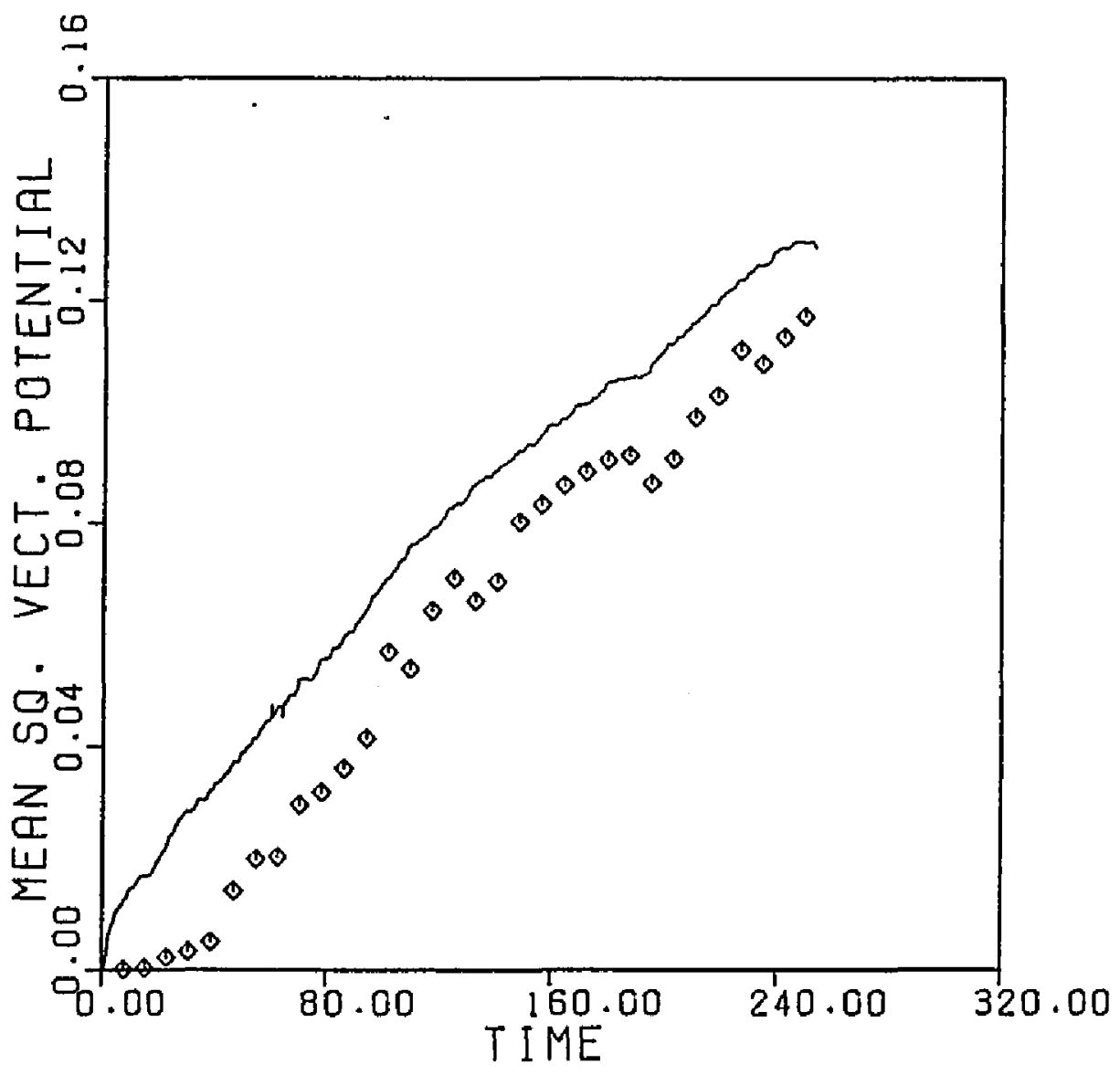


FIGURE 9A

VECTOR POTENTIAL SPECTRUM

AVERACED OVER 2000 TIME STEPS, ENDED AT $r=19.53$

DRIVEN MHD RUN

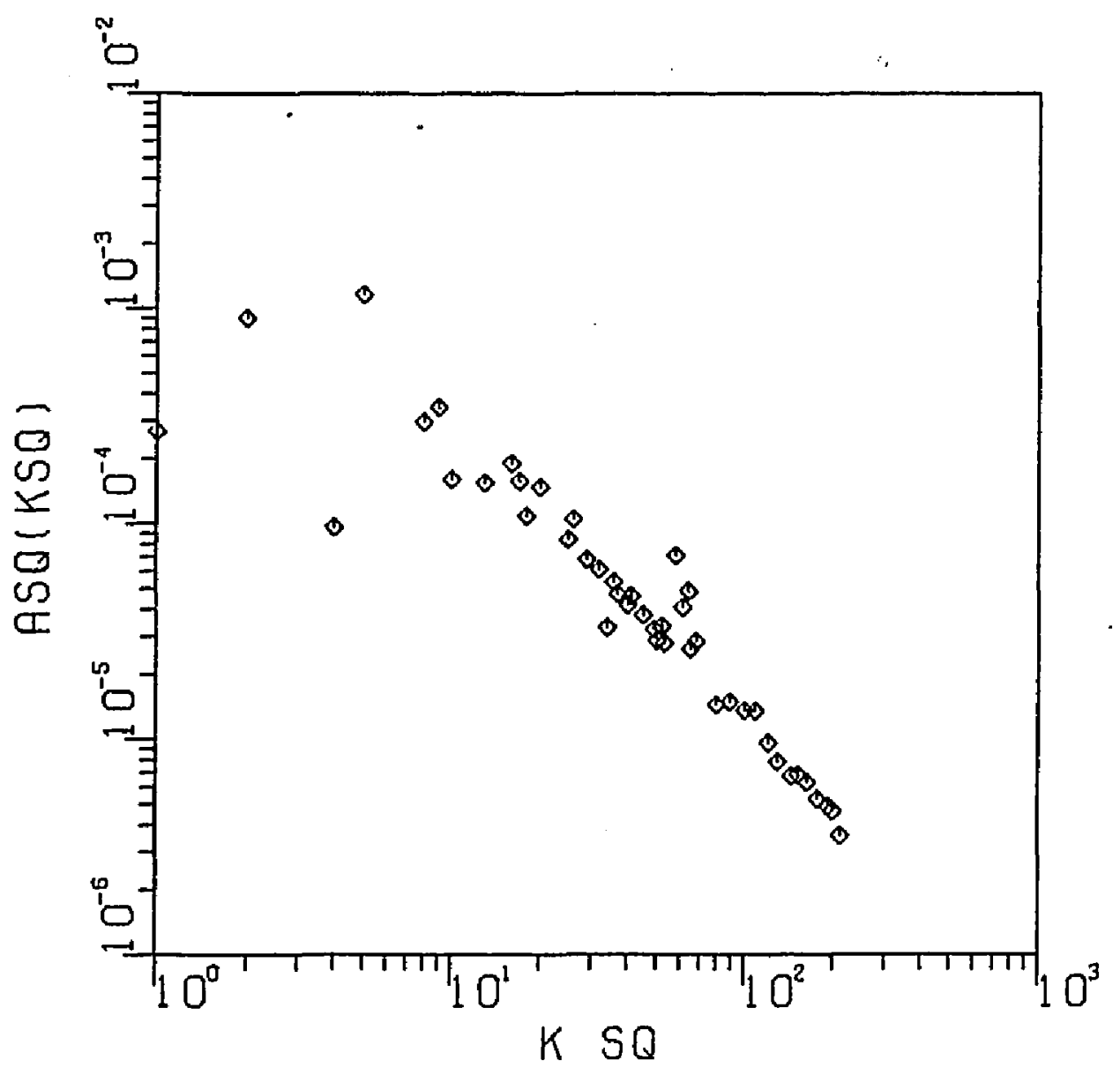


FIGURE 9B

VECTOR POTENTIAL SPECTRUH

AVERAGED OVER 2000 TIME STEPS. ENDED AT $T=136.32$

DRIVEN MHO RUN

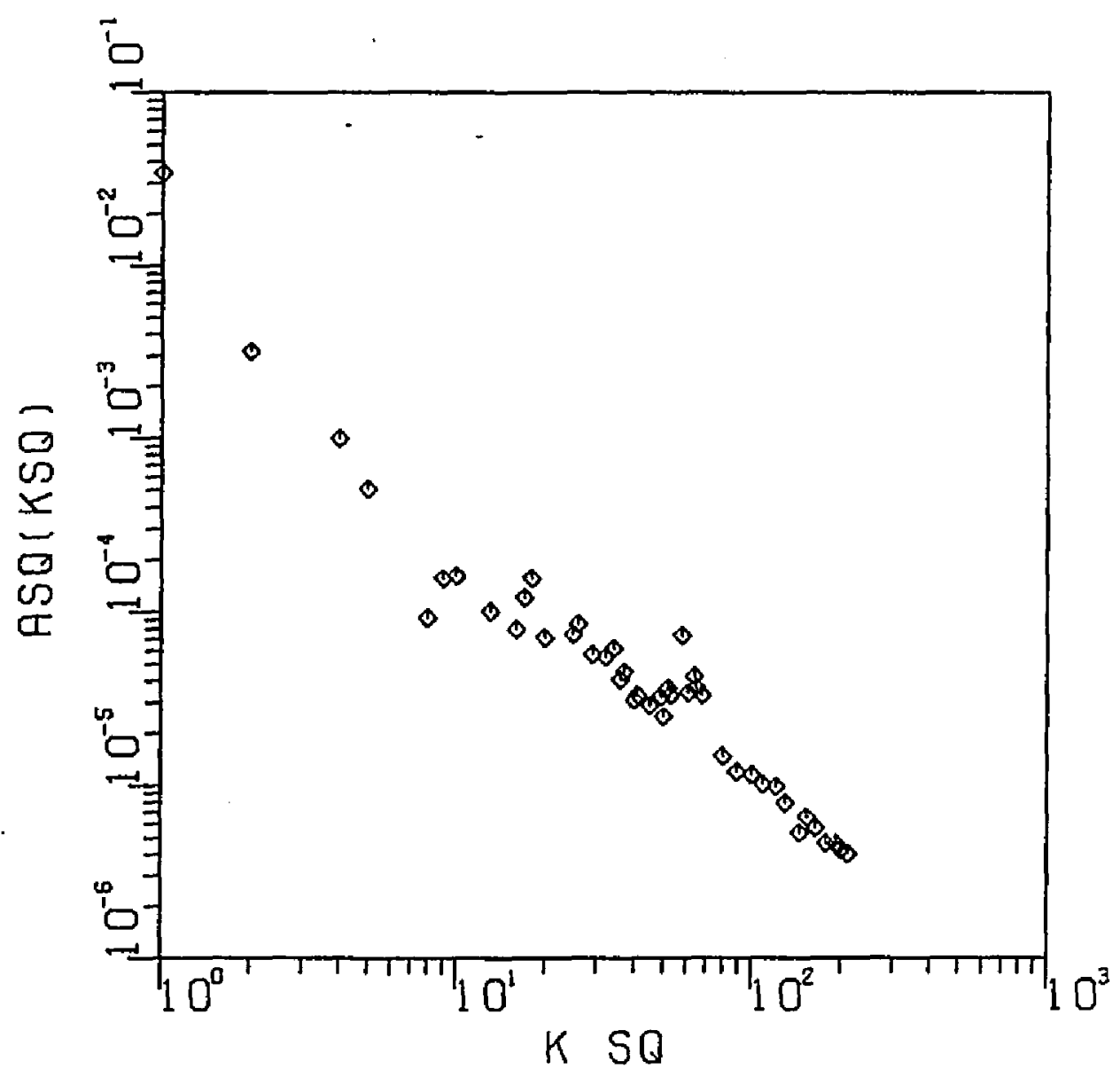


FIGURE $\quad 9 C$

VECTOR POTENTIAL SPECTRUH

AVERACED OVER 2000 TIHE STEPS, ENDED AT $T=253.91$

DRIVEN MHD RUN

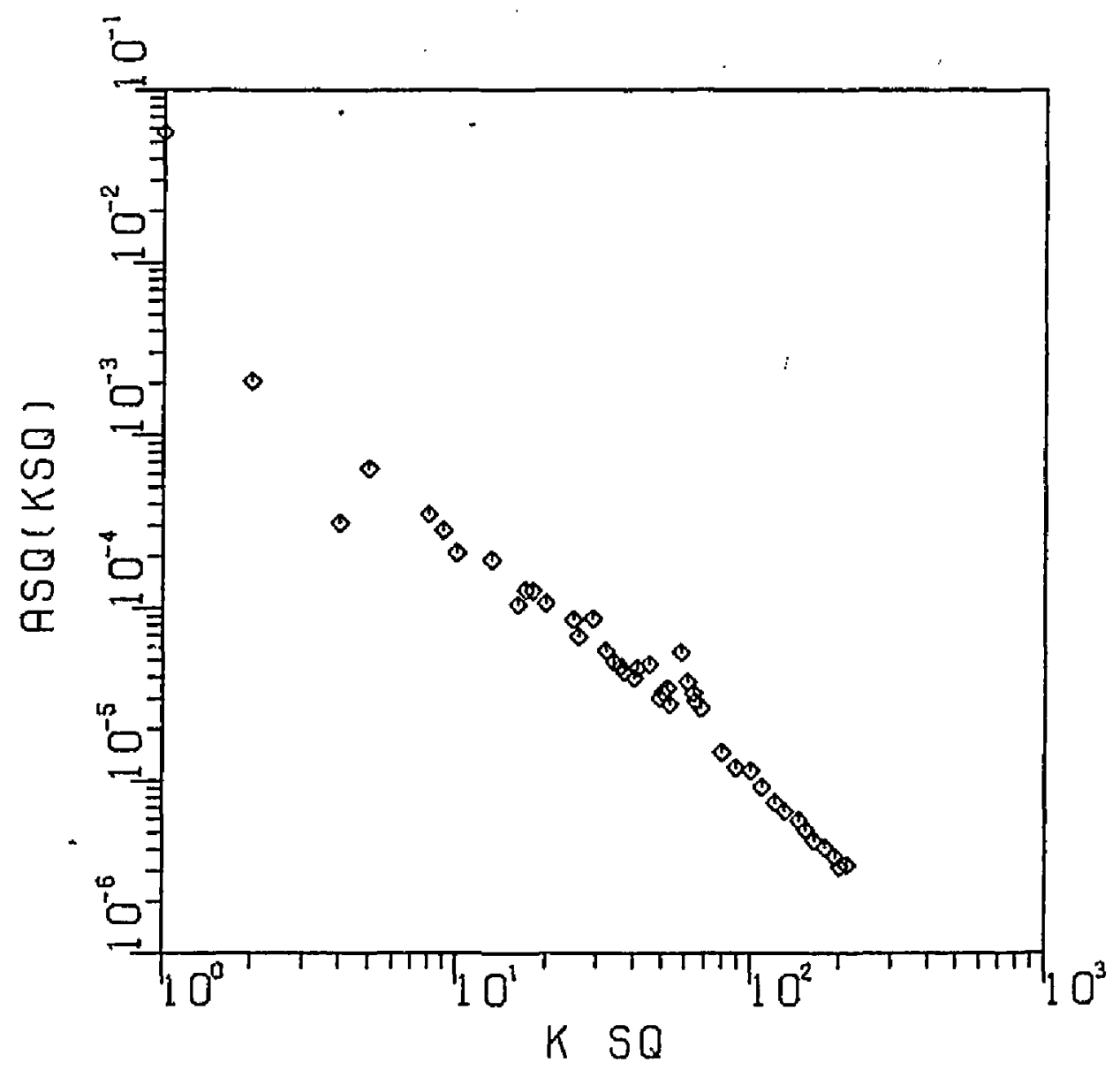


FIGURE $9 D$

HECHANICAL HODAL ENERGY SPECTRUM

AVERACED OVER 2000 TIME STEPS. ENOED AT $T=19.53$

ORIVEN MHD RUN

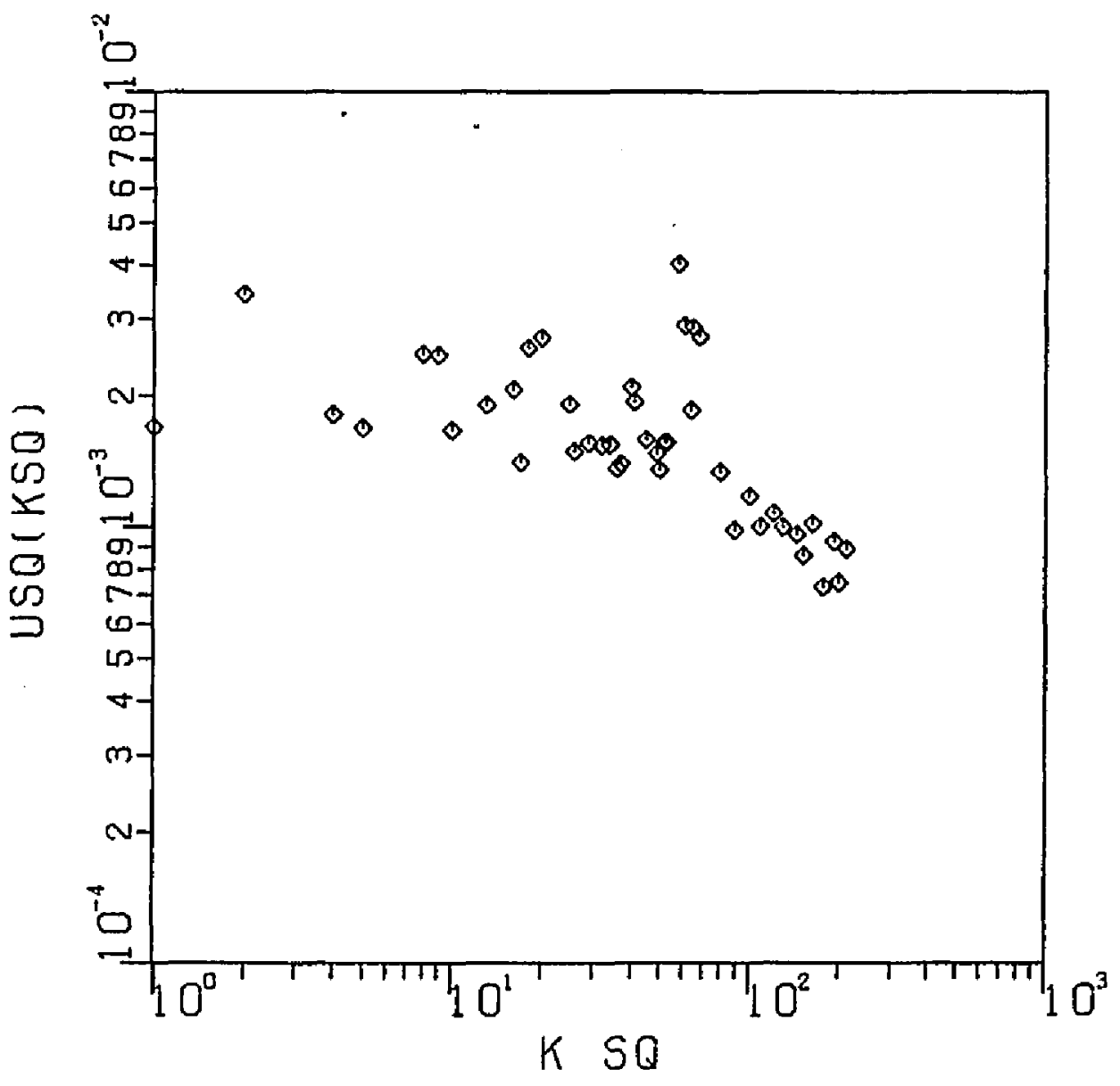


FIGURE GE

MECHANICAL MODAL ENERGY SPECTRUM

AVERACED OVER 2000 TIME STEPS. ENDEO AT $T=136.72$

ORIVEN MHD RUN

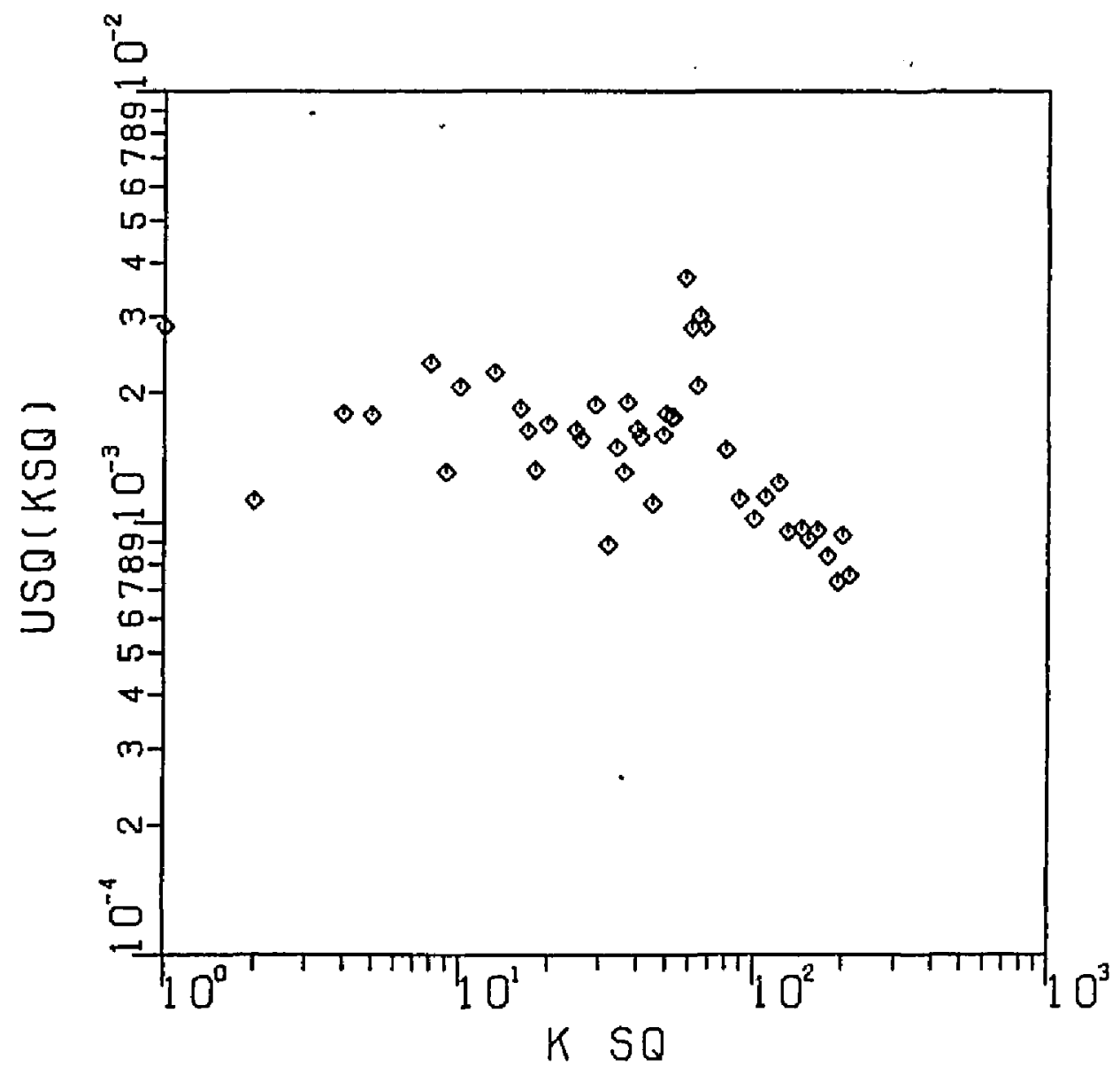




\section{FIGURE 9F}

MECHANICAL MOOAL ENERCY SPECTRUM

AVERAGED OVER 2000 TIME STEPS, ENDED AT $T=253.91$

DRIVEN MHD RUN

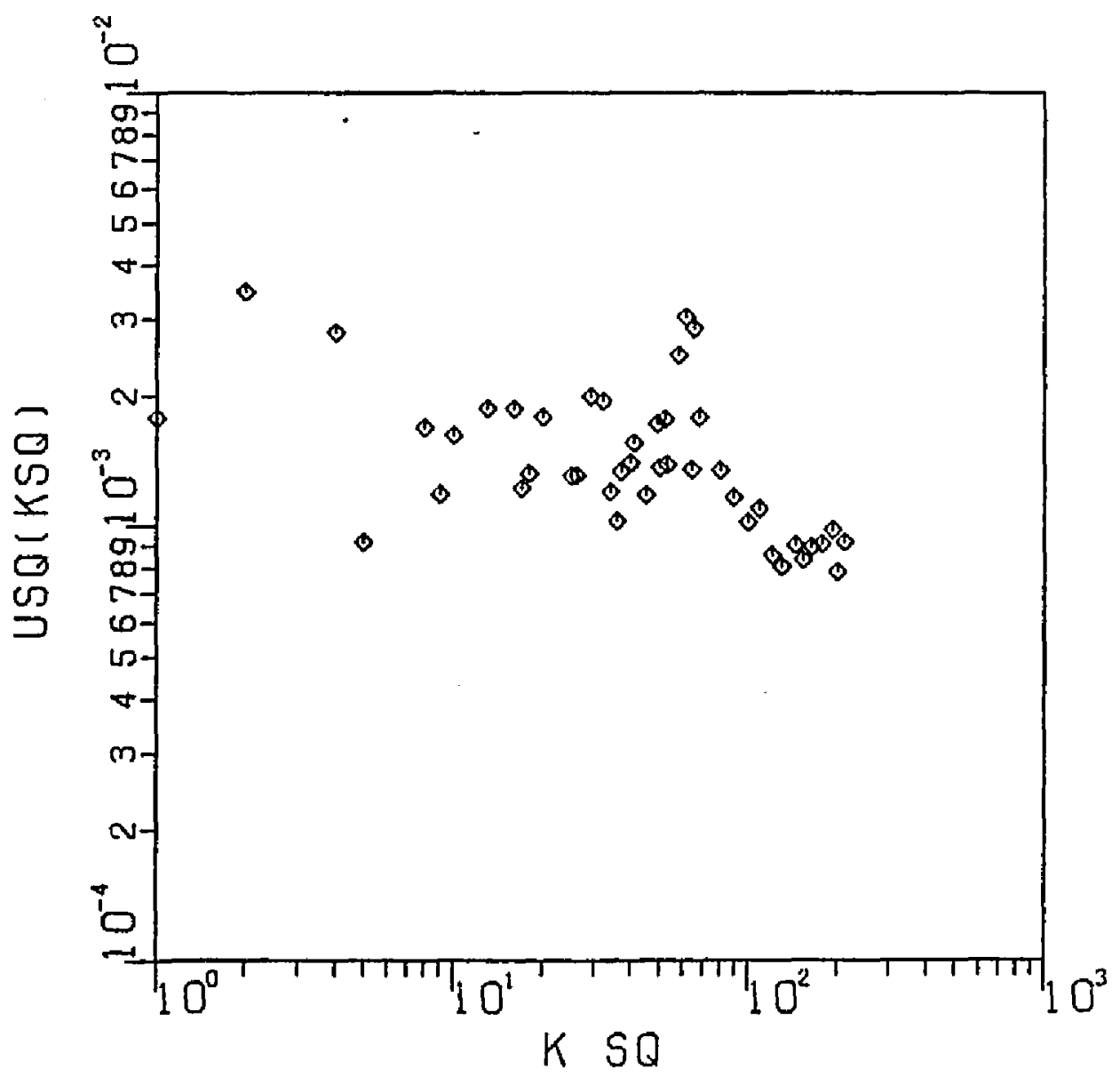




\section{FIGURE $\quad 9 G$}

MACNETIC MOOAL. ENERGY SPECTRUH

AVERACED OVER 2000 TIHE STEPS. ENDED AT $T=19.53$

GRIVEN MHD RUN

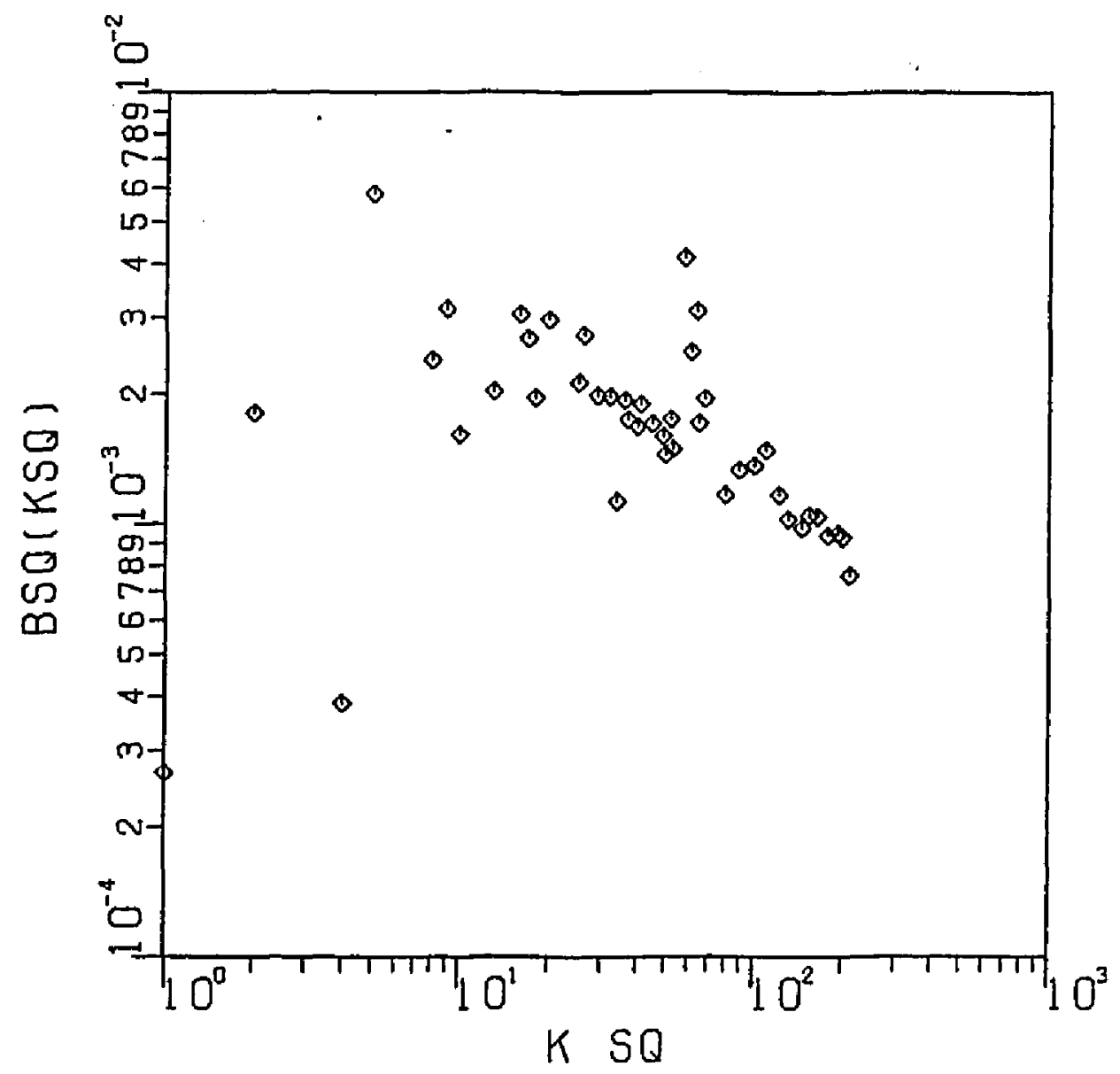


FIGURE $\quad 9 H$

MAGNETIC MODAL ENERGY SPECTRUH

AVERAGEO OVER 2000 TIME STEPS. ENDED AI $T=136.72$

DRIVEN MHD RUN

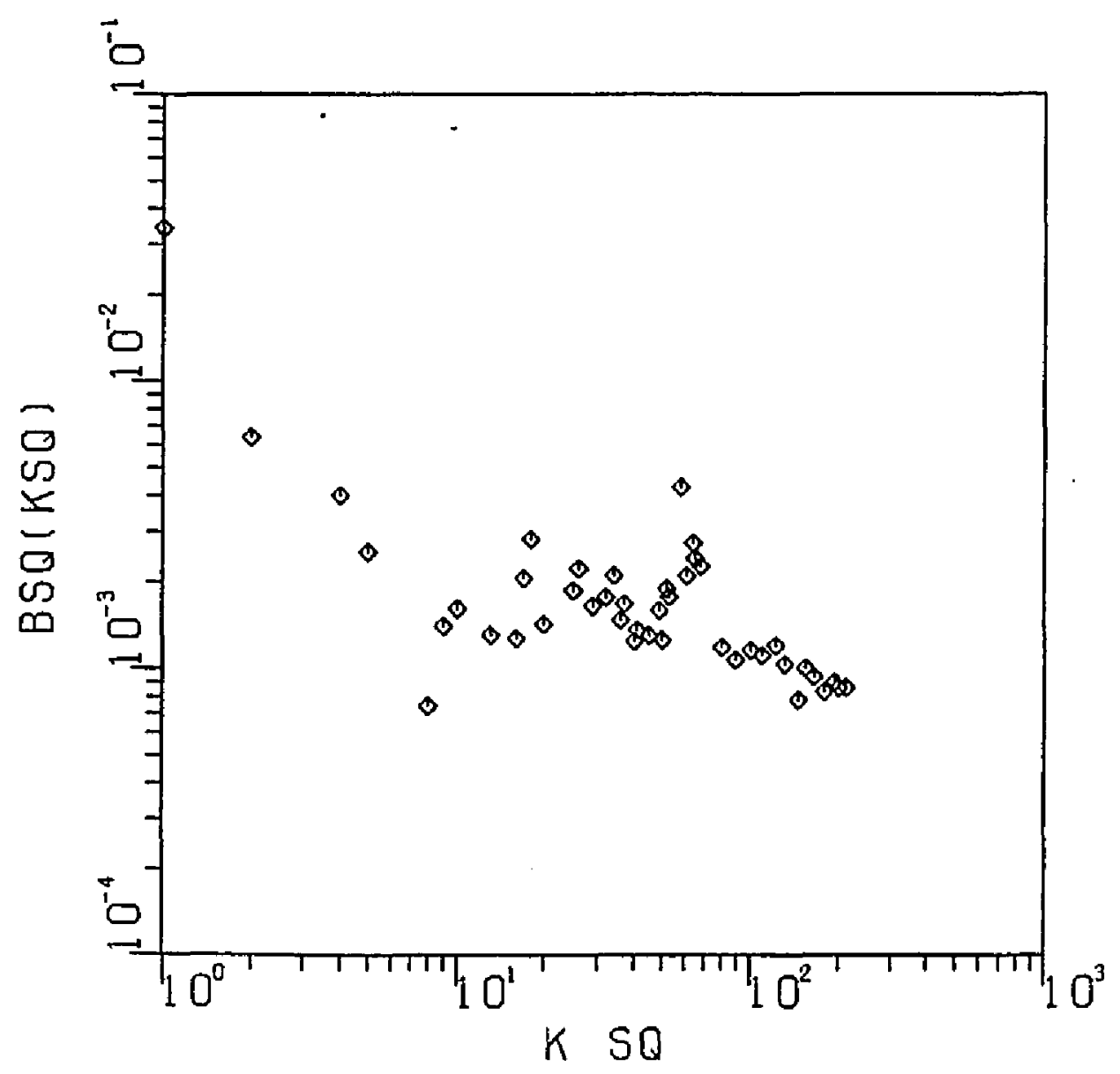


FIGURE 9 I

MAGNETIC MODAL ENERGY SPECTRUH

AVERAGED OVER 2000 TIME STEPS, ENOED AT $T=253.91$

DRIVEN MHO RUN

SOLID LINES ARE

POHER LAH LEAST SOUARE FIT

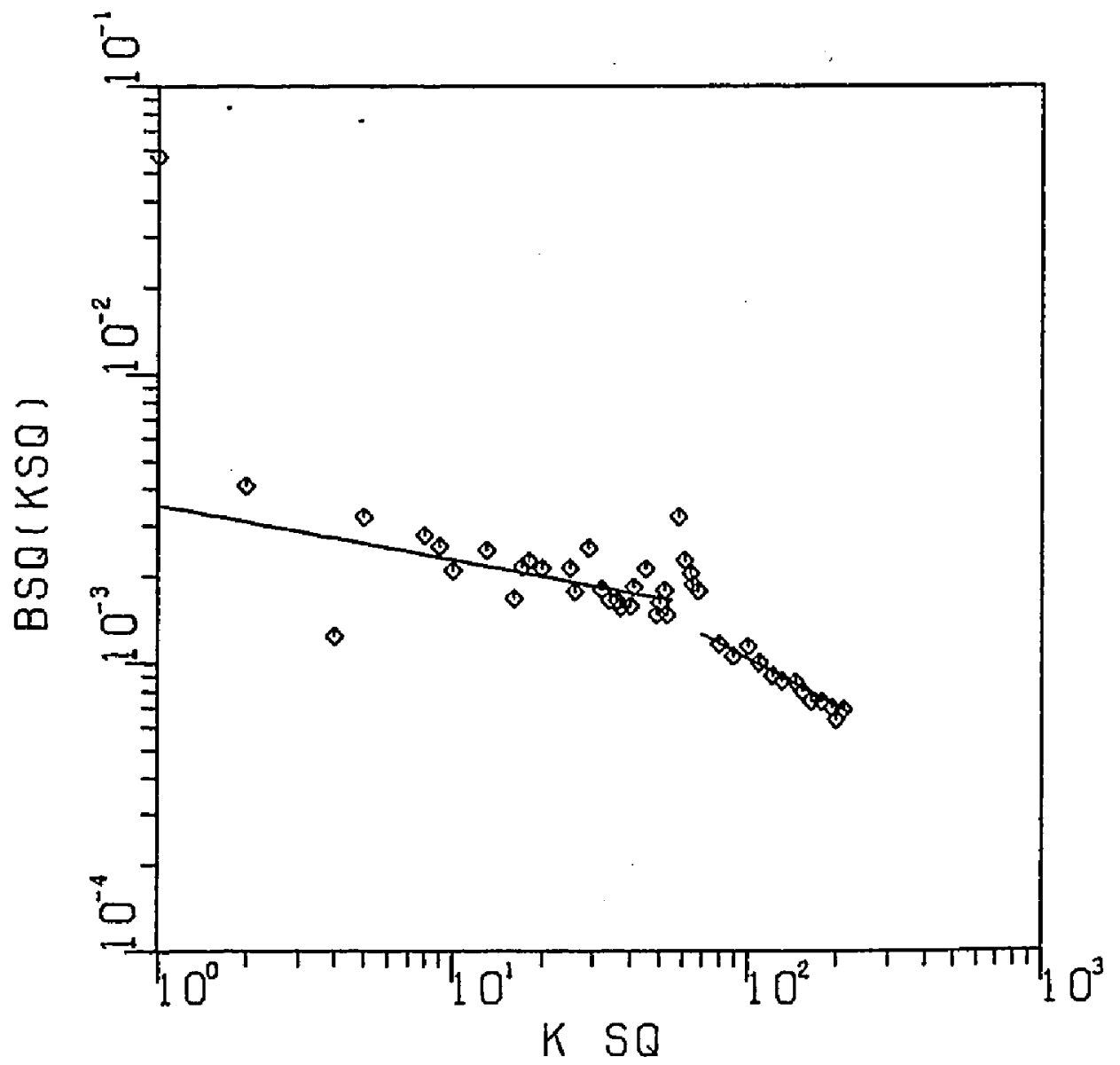




\section{F IGURE $10 A$}

CONTOURS OF STREAM FUNCTION

RT $T=19.53$, TIHE STEP 5000

ORIVEN MHO RUN

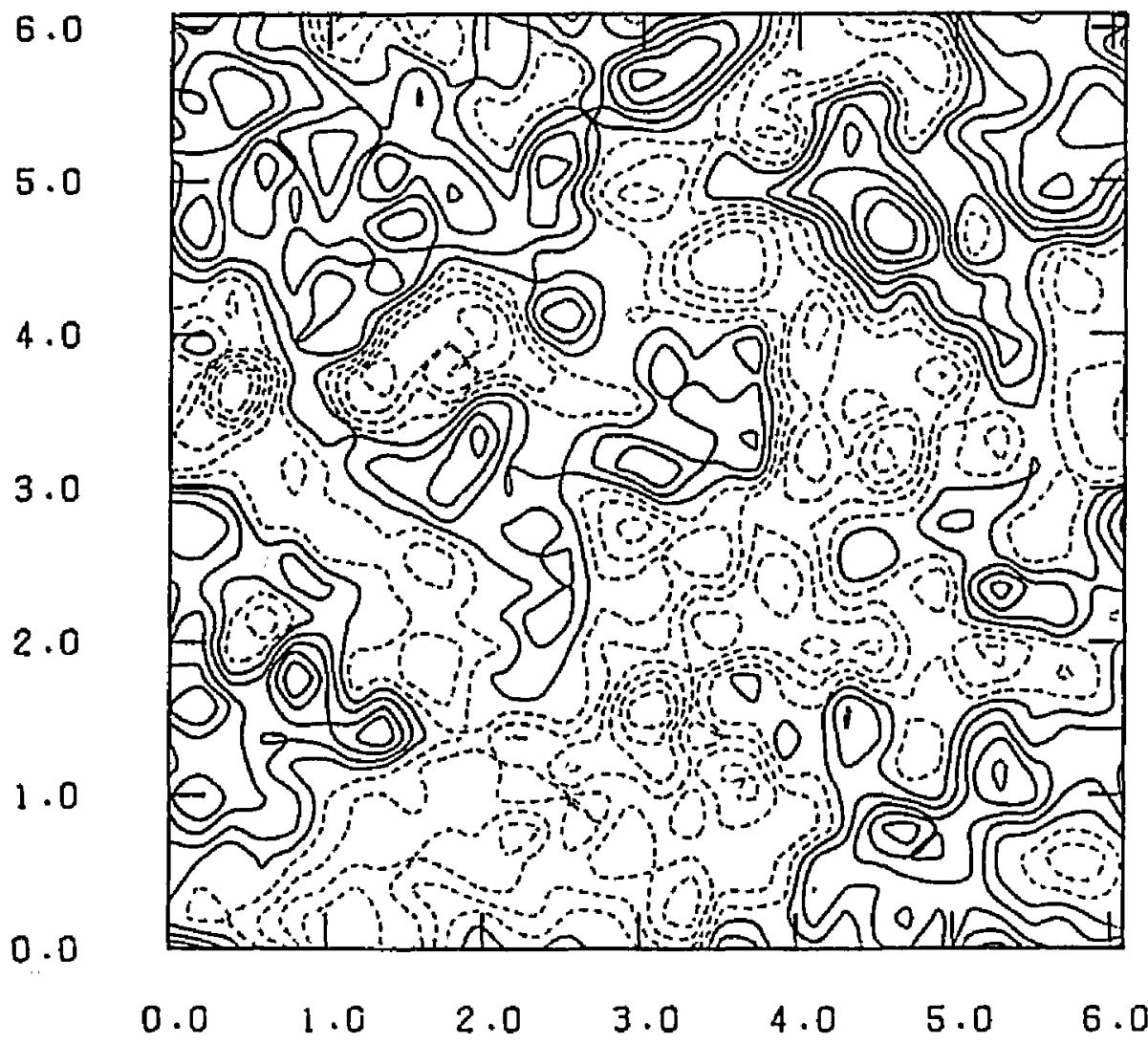




\section{F I GURE $10 B$}

CONTOURS OF STREAH FUNCTION

AT $T=136.72$. TIME STEP 35000

DRIVEN MHO RUN

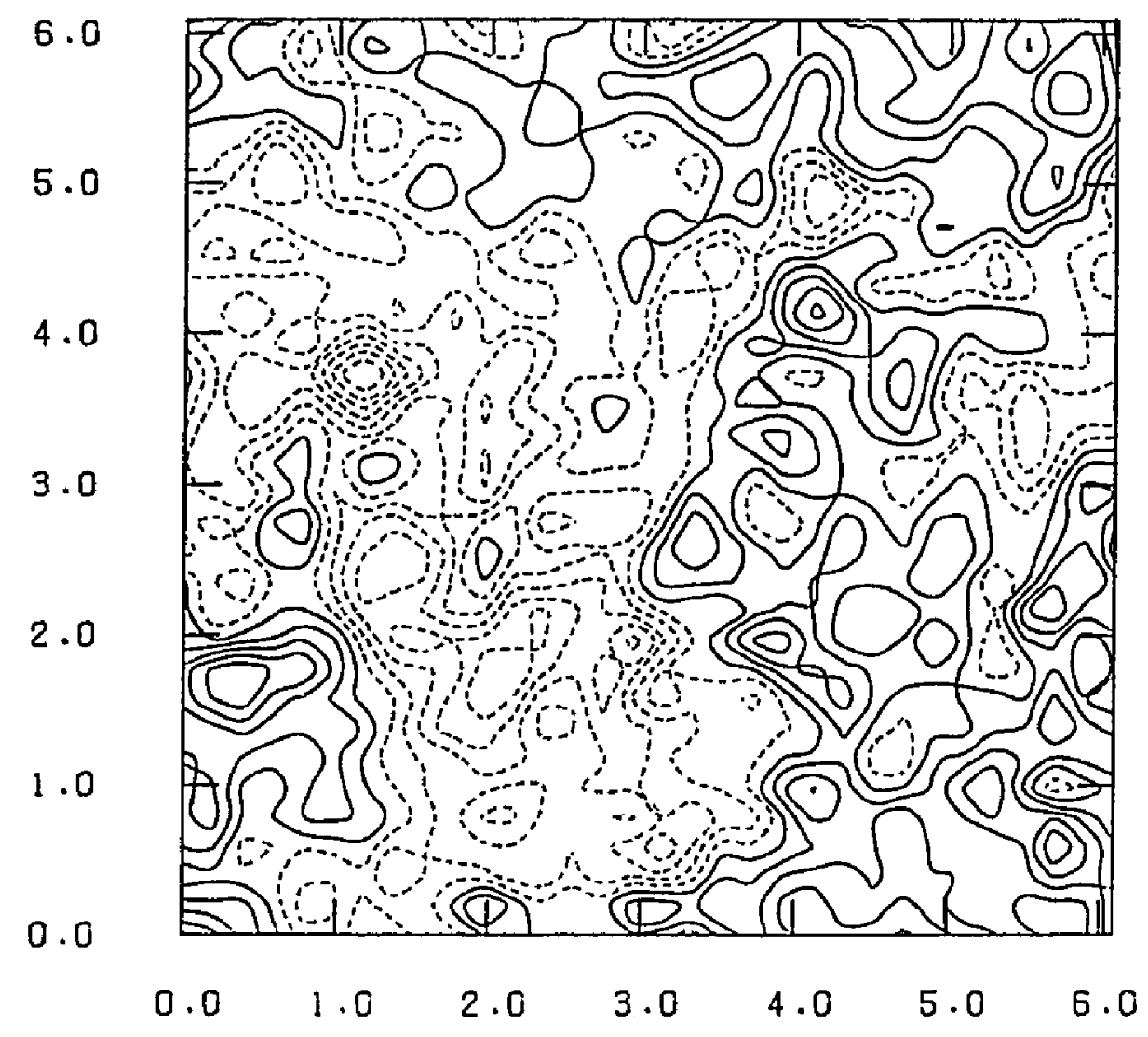


F I GURE $10 \mathrm{C}$

CONTOURS OF STREAH FUNCTION

AT $T=253.90$. TIME STEP 65000

DRIVEN MHD RUN

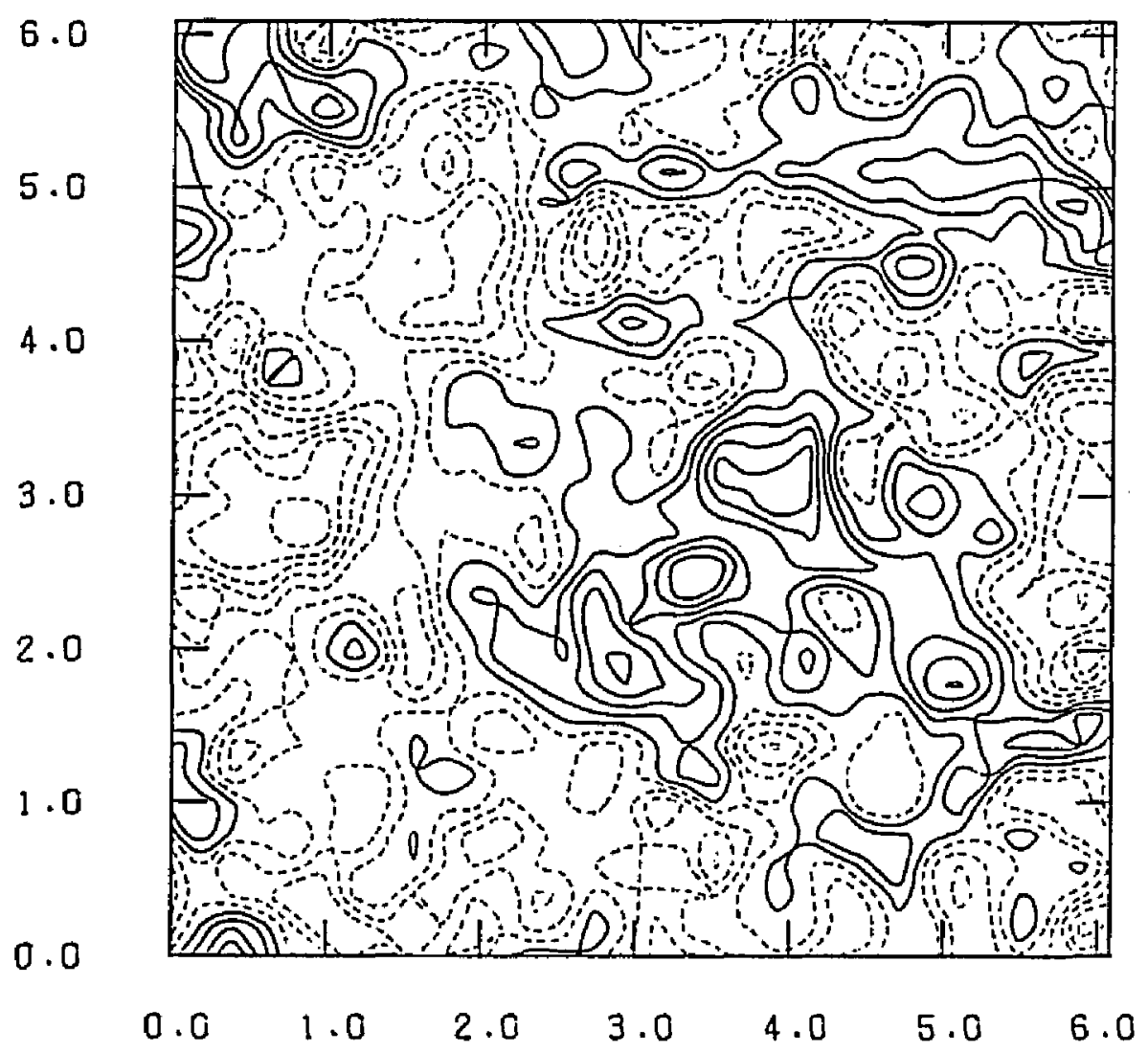


F I GURE 100

CONTOURS OF VORTICITY

AT $T=19.53$. TIME STEP 5000

DRIVEN MHD RUN

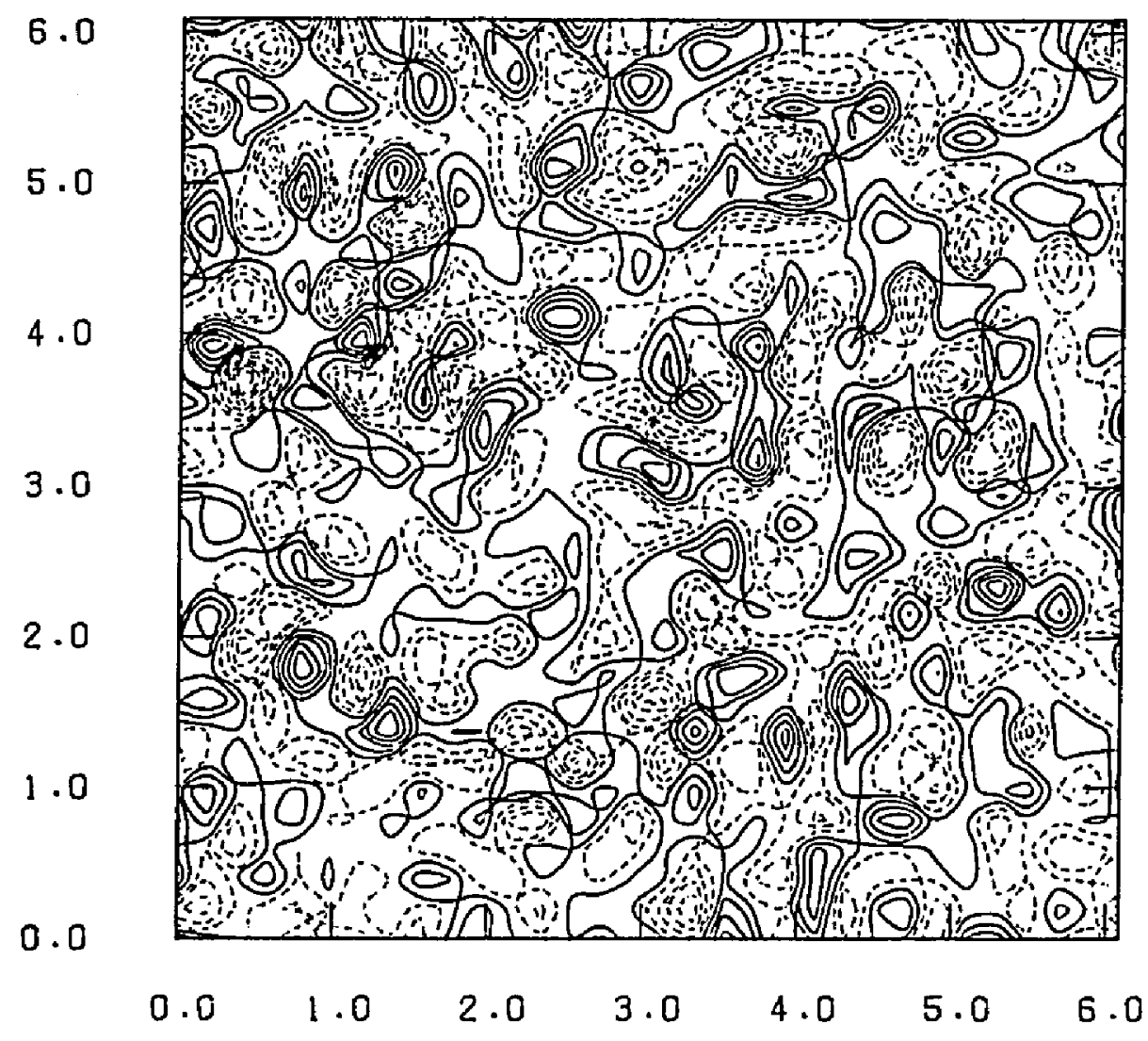




\section{F I GURE $10 E$}

CONTOURS OF VORTICITY

AT $T=136.72$, TIME STEP 35000

DRIVEN MHD RUN

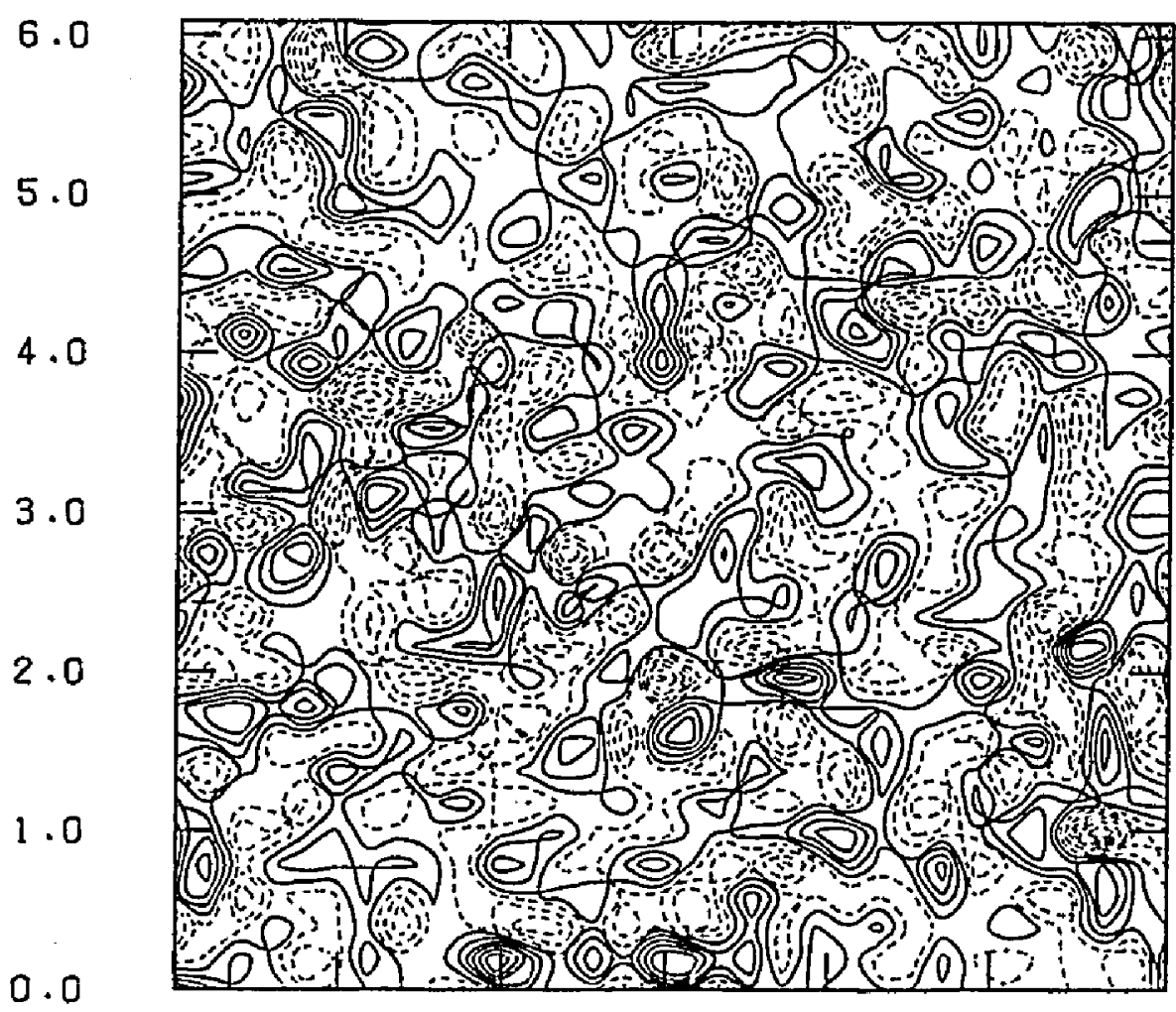

$\begin{array}{lllllll}0.0 & 1.0 & 2.0 & 3.0 & 4.0 & 5.0 & 6.0\end{array}$ 


\section{F IGURE 1 OF}

CONTOURS OF VORTICITY

AT $T=253.90$, TIME STEP 65000

ORIVEN MHID RUN

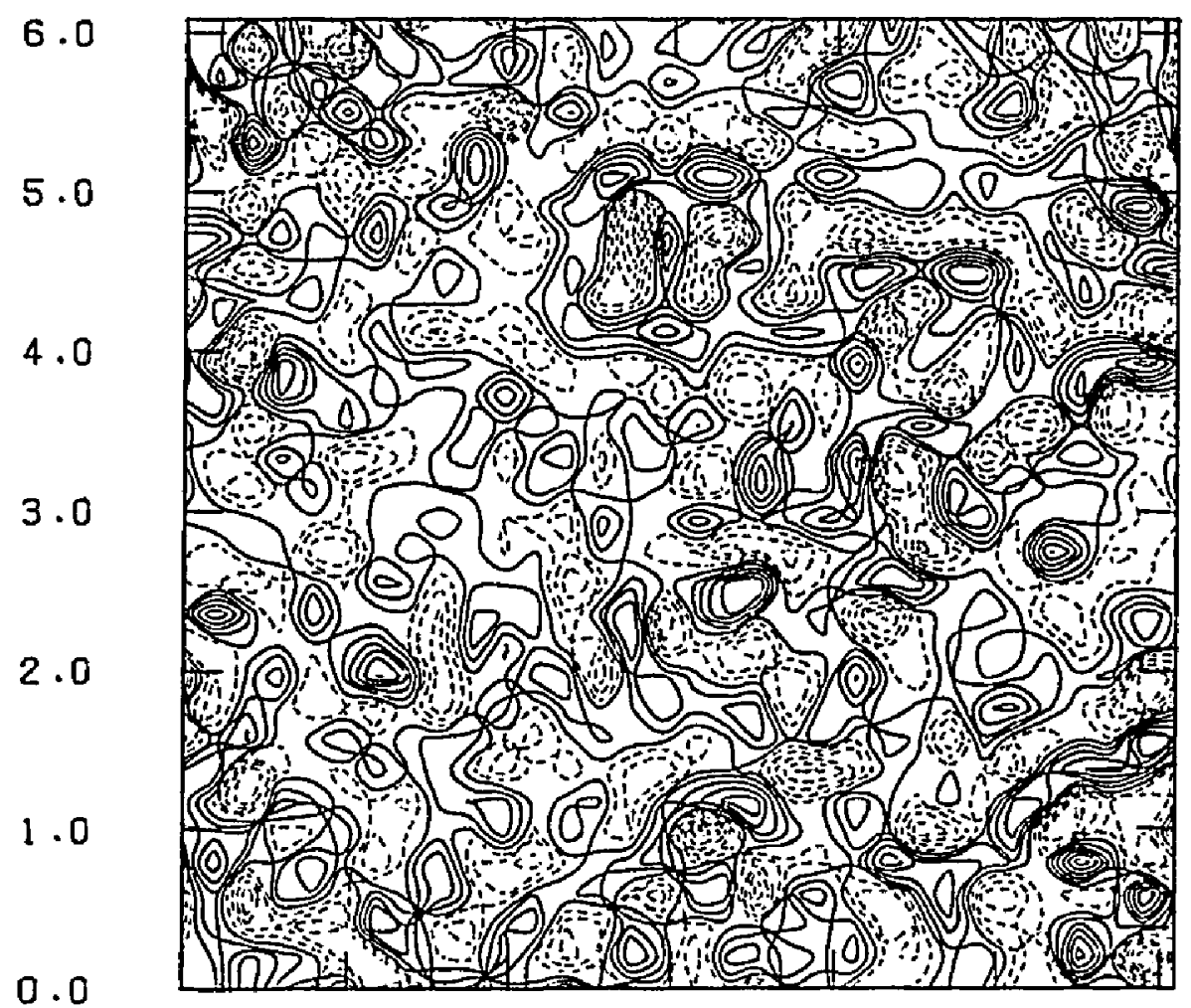

$\begin{array}{lllllll}0.0 & 1.0 & 2.0 & 3.0 & 4.0 & 5.0 & 6.0\end{array}$ 
FIGURE $10 G$

CONTOURS OF VECTOR POTENTIRL

AT $T=19.53$, TIME STEP 5000

DRIVEN MHD RUN

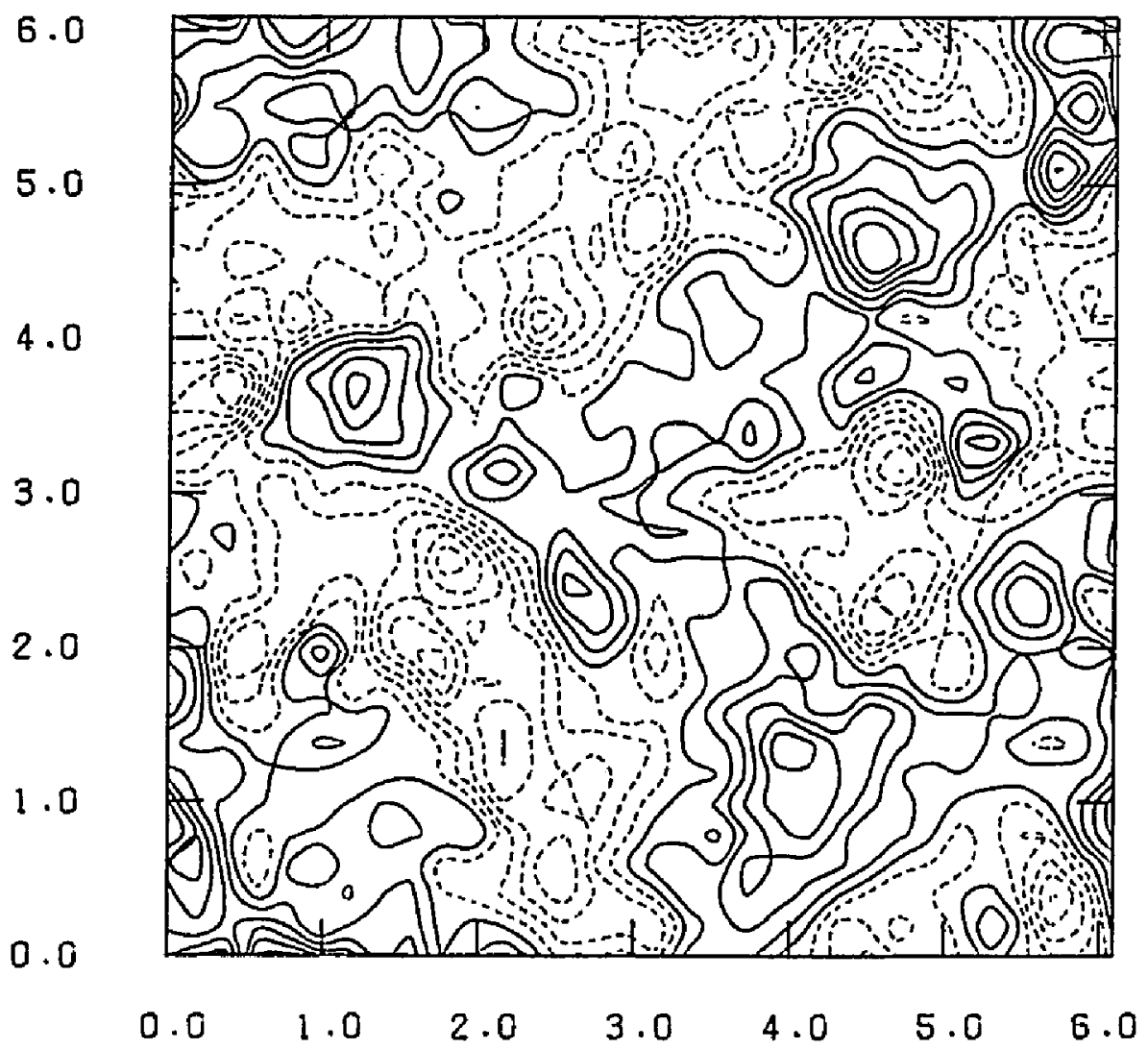




\section{F I GURE $10 \mathrm{H}$}

CONTOURS OF VECTOR POTENTIAL

AT $T=136.72$. TIME STEP 35000

DRIVEN HHO RUN

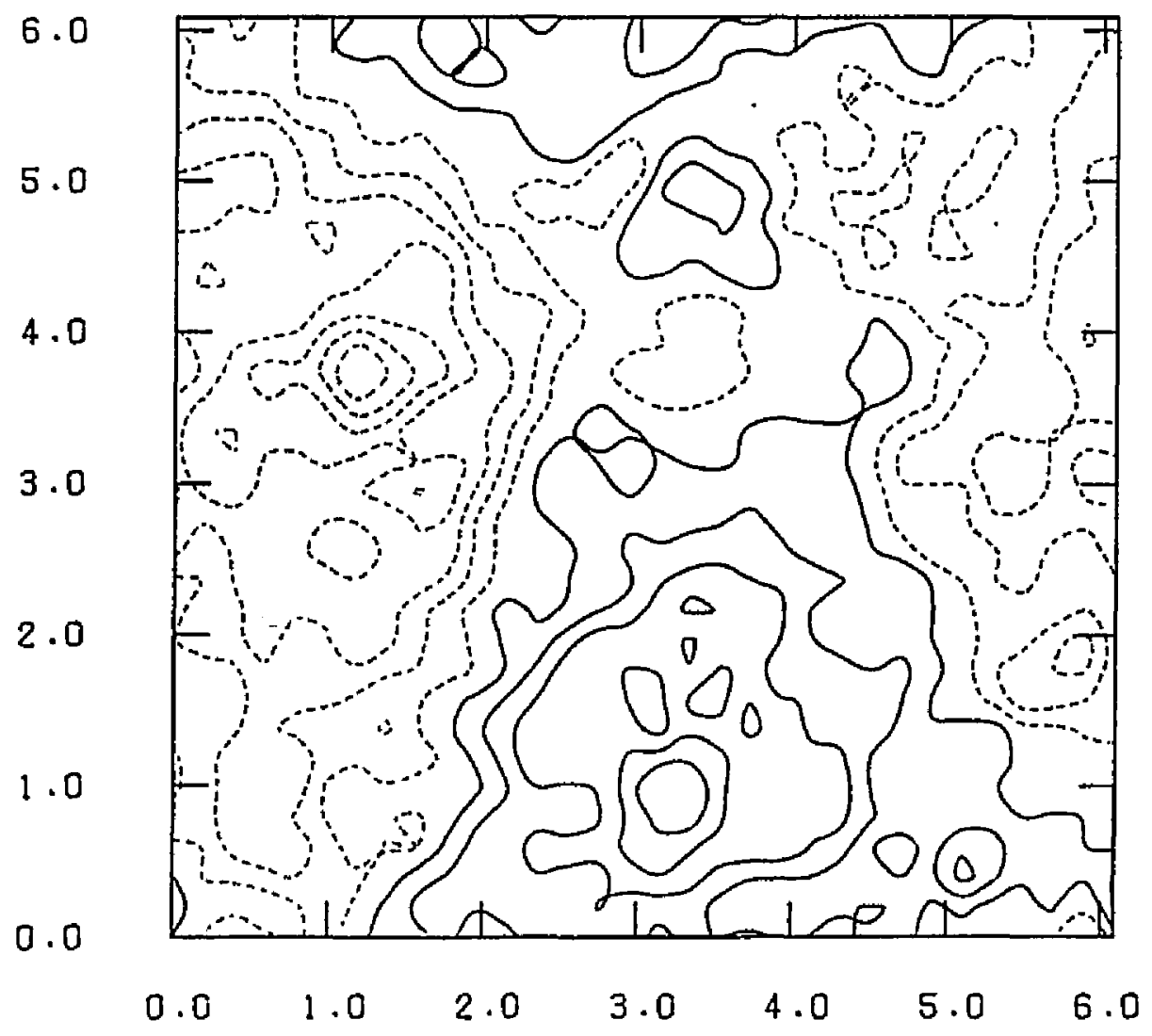


F I GURE 10 I

CONTOURS OF VECTOR POTENTIAL

AT $T=253.90$. TIME STEP 65000

DRIVEN MHO RUN

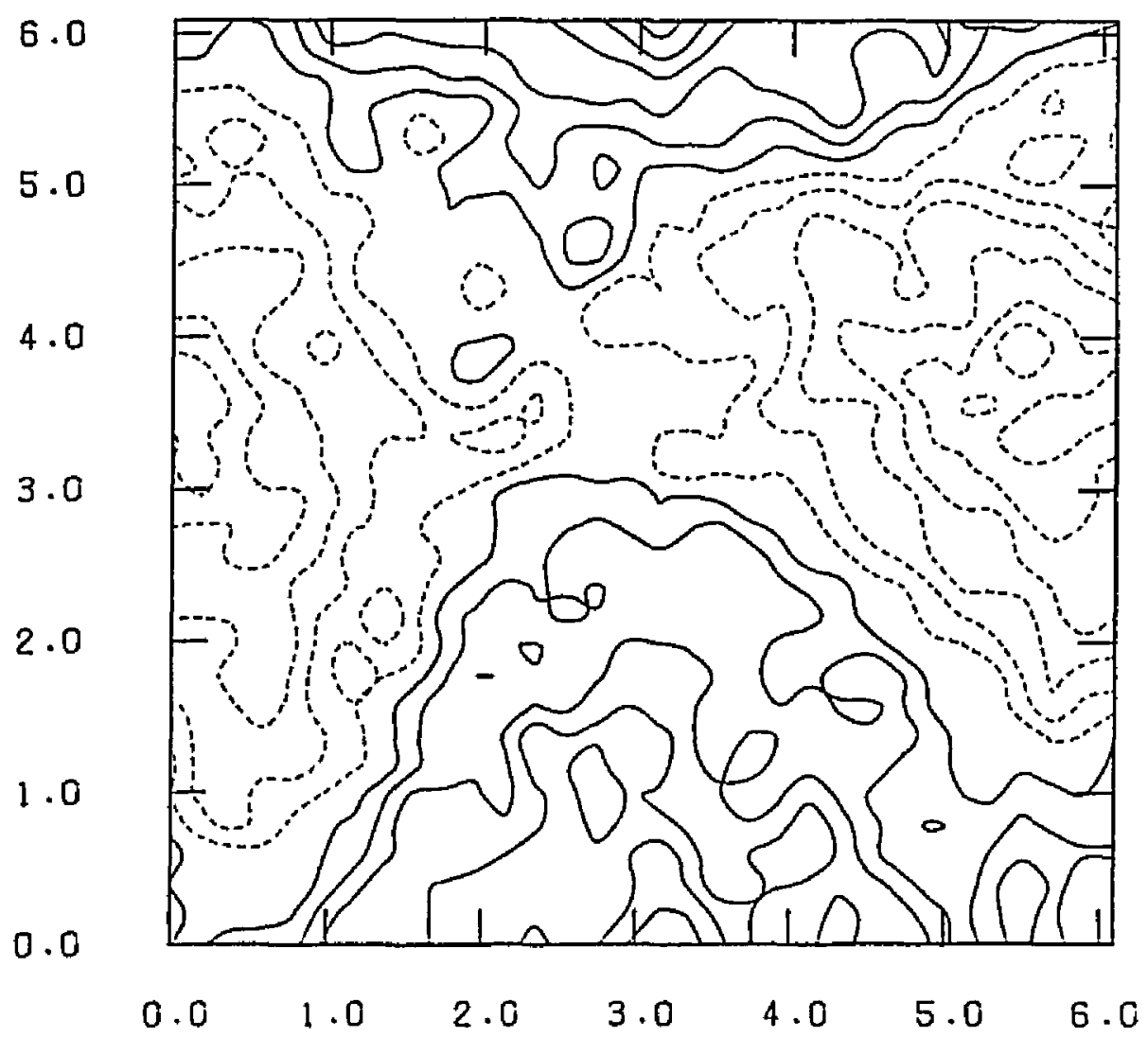




\title{
FIGURE $10 \mathrm{~J}$
}

\author{
CONTOURS OF CURRENT DENSITY \\ AT $T=19.53$. TIME STEP 5000 \\ ORIVEN MHD RUN
}

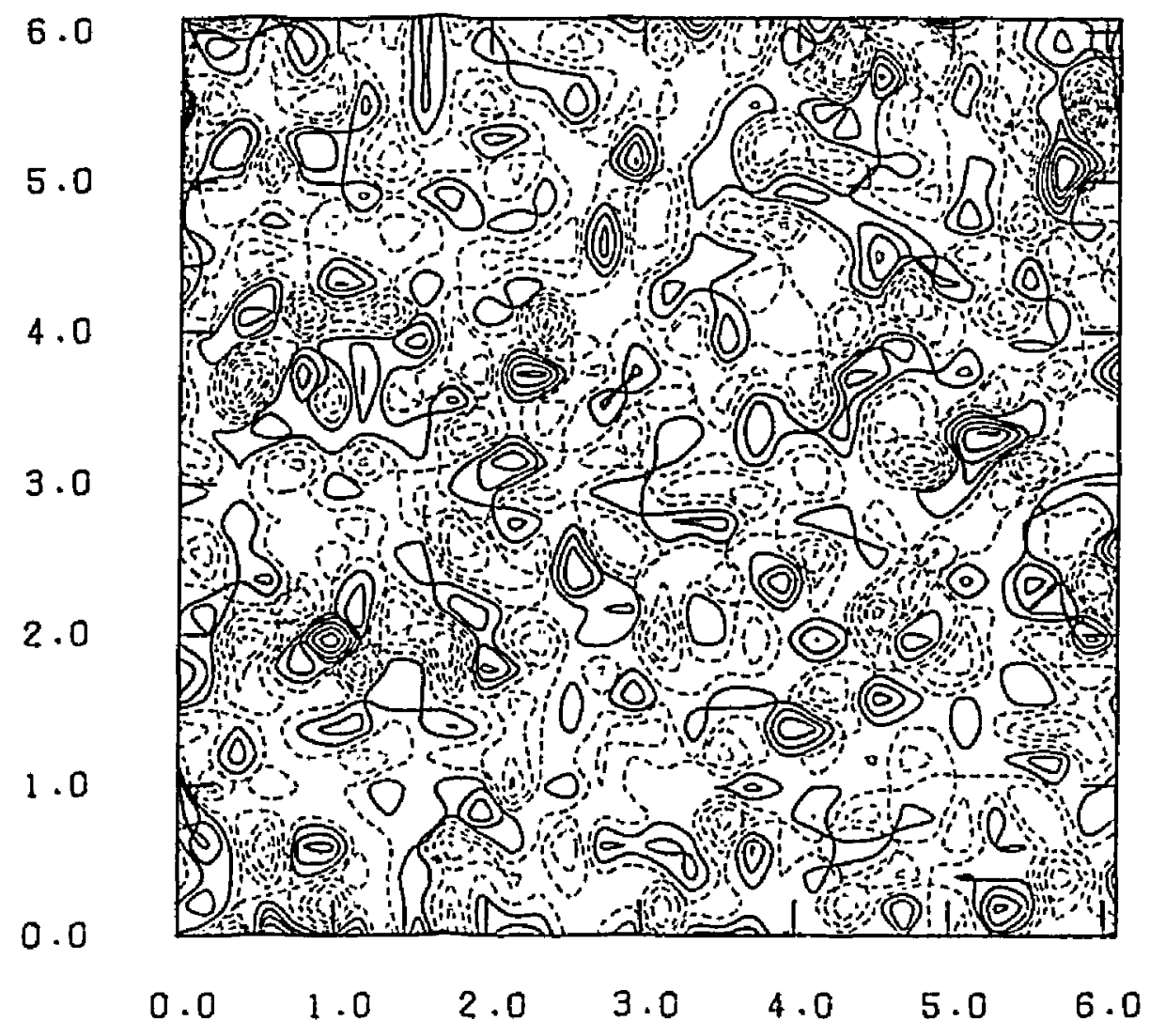




\section{FIGURE 1OK}

CONTOURS OF CURRENT DENSITY

AT $T=136.72$. TIME STEP 35000

ORIVEN HHD RUN

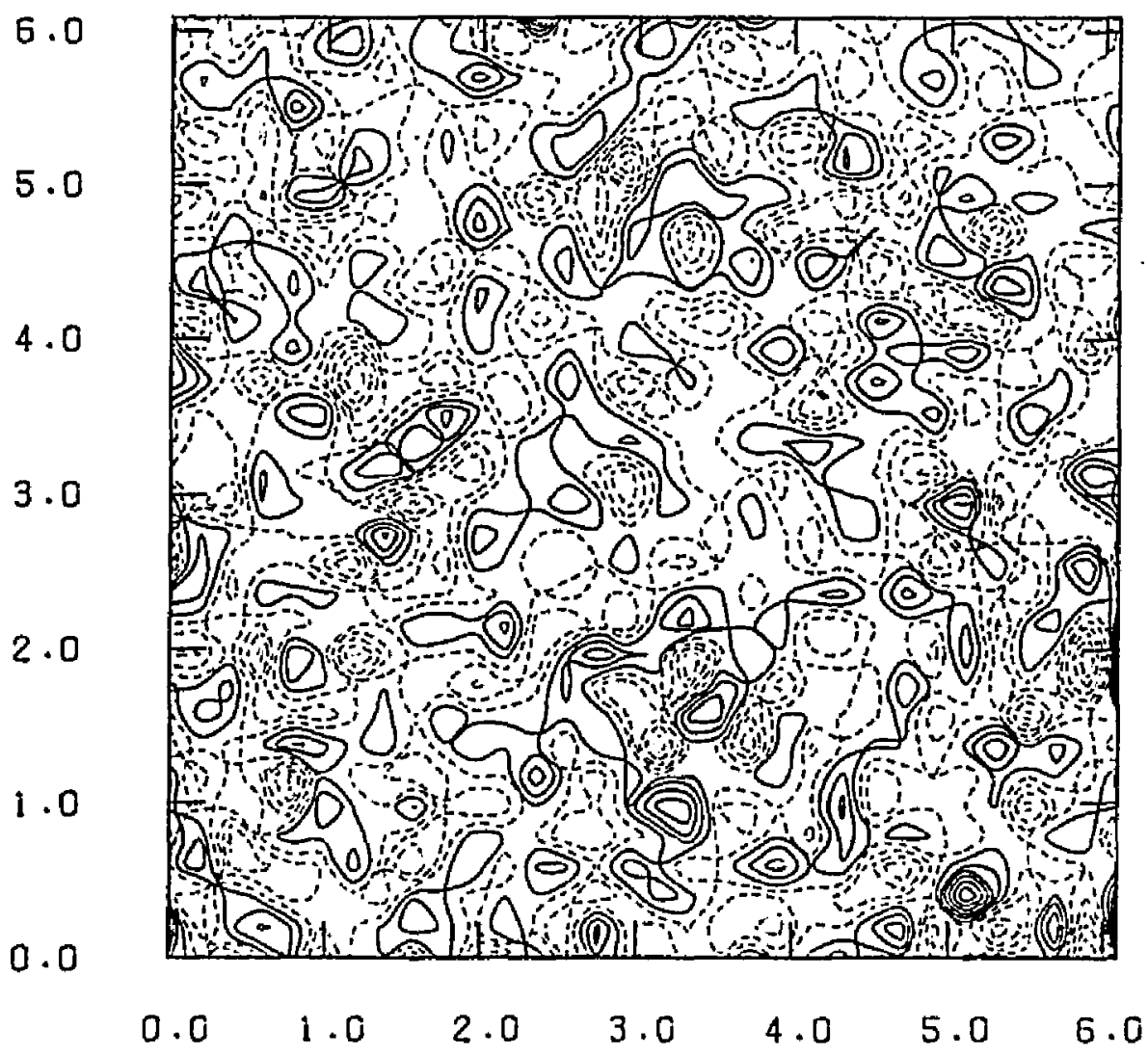




\section{F IGURE $10 L$}

CONTOURS OF CURRENT DENSITY

AT $T=253.90$, TIME STEP 65000

DRIVEN MHD RUN

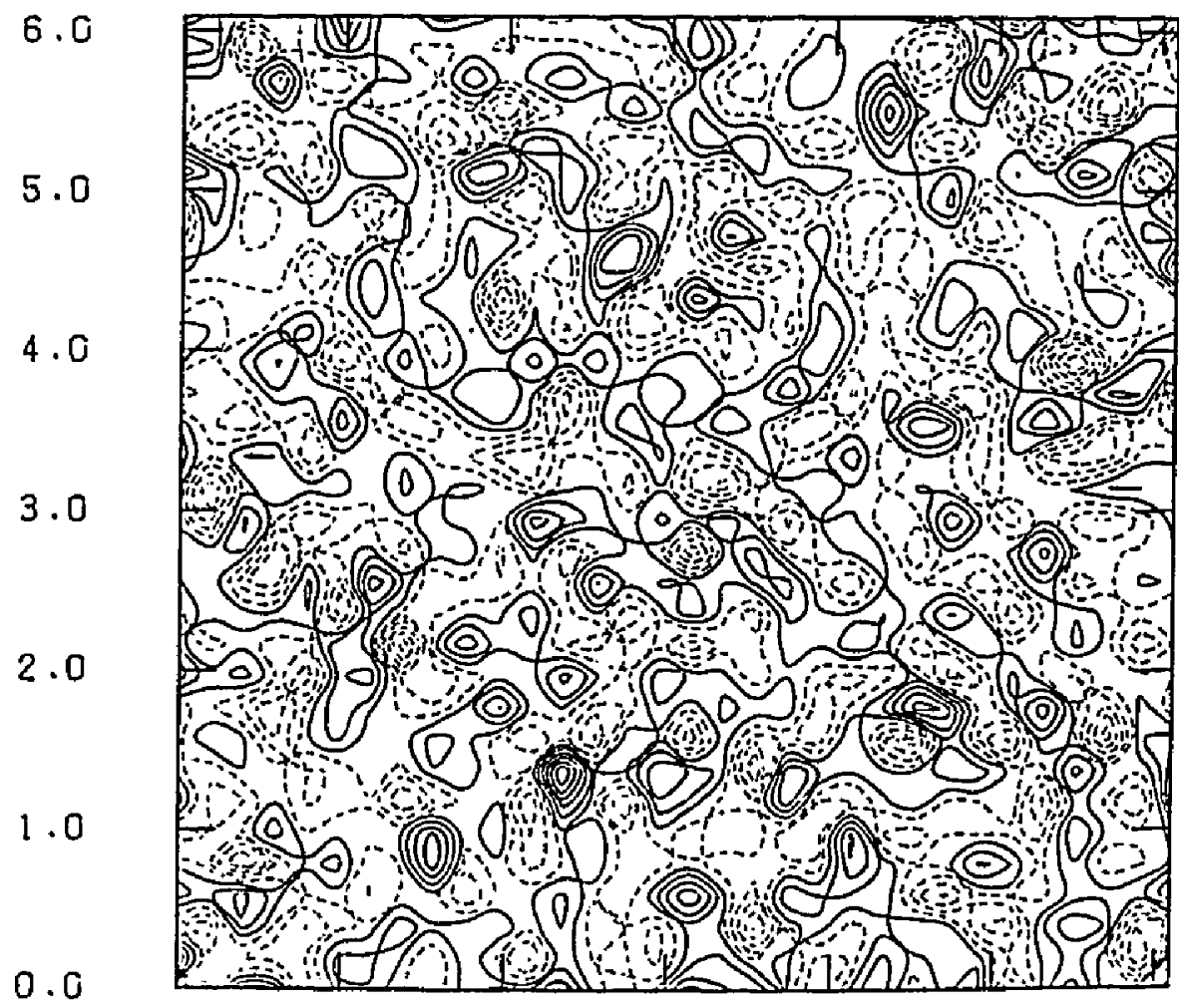

$\begin{array}{lllllll}0.0 & 1.0 & 2.0 & 3.0 & 4.0 & 5.0 & 6.0\end{array}$ 


\section{F IGURE $11 \mathrm{~A}$}

ENERGY VERSUS TIME

DRIVEN MHD (STAR) RUN

MAGNETIC CONTRIBUTION LABELED AS B

MECHANICAL CONTRIBUTION LABELED AS Y

RRROH SHOHS HHEN SHITCHED TO GAXGA GRID

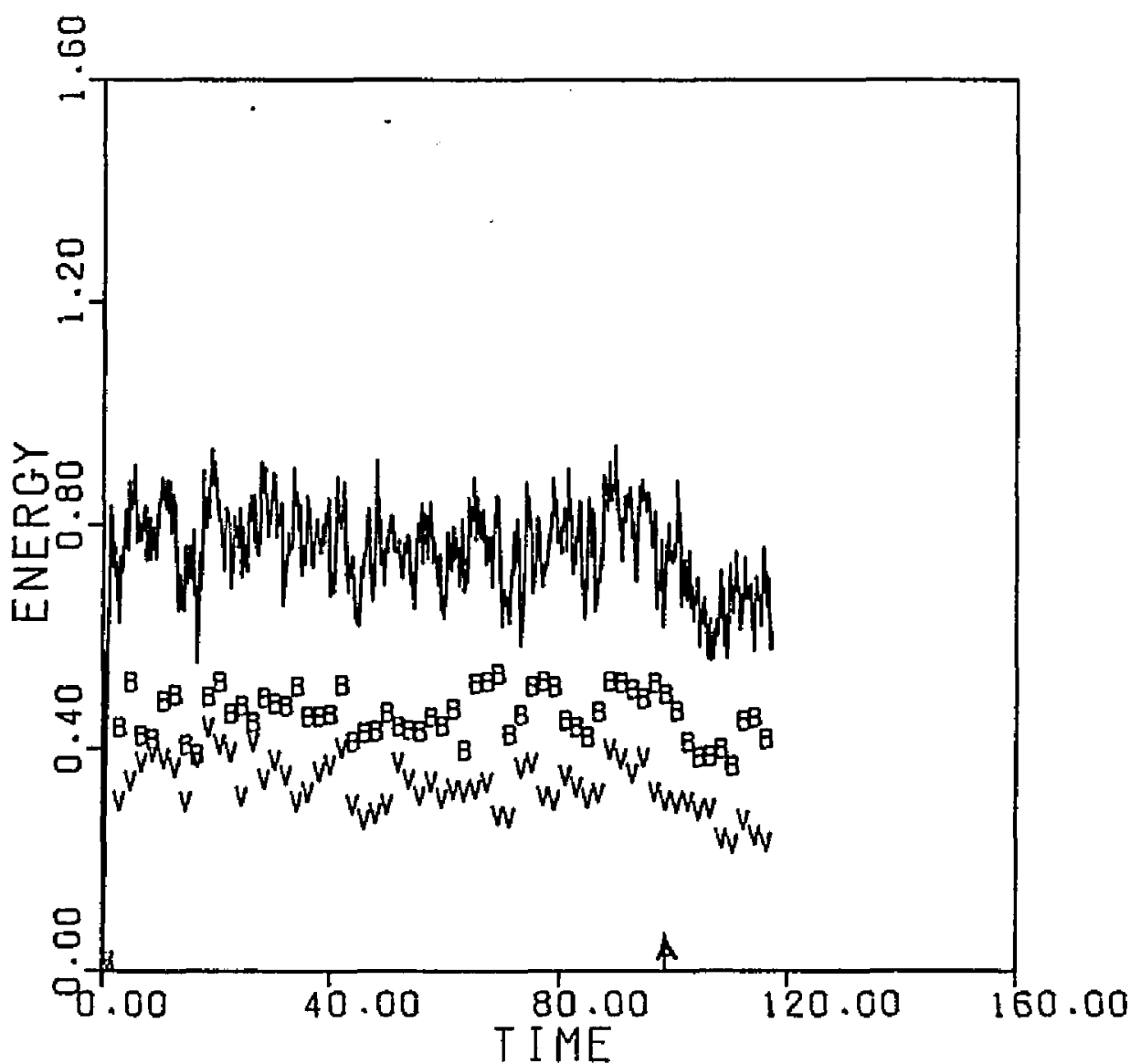




\section{F IGURE $11 \mathrm{~B}$}

MEAN SOUARE VECTOR POTENTIAL VERSUS TIHE

DRIVEN HHD (STAR) RUN

SOUARES REPRESENT THE CONTRIBUTION DUE TO THE FUNDAMENTAL

ARROH SHOHS HHEN SHITCHED TO 64X64 GRID

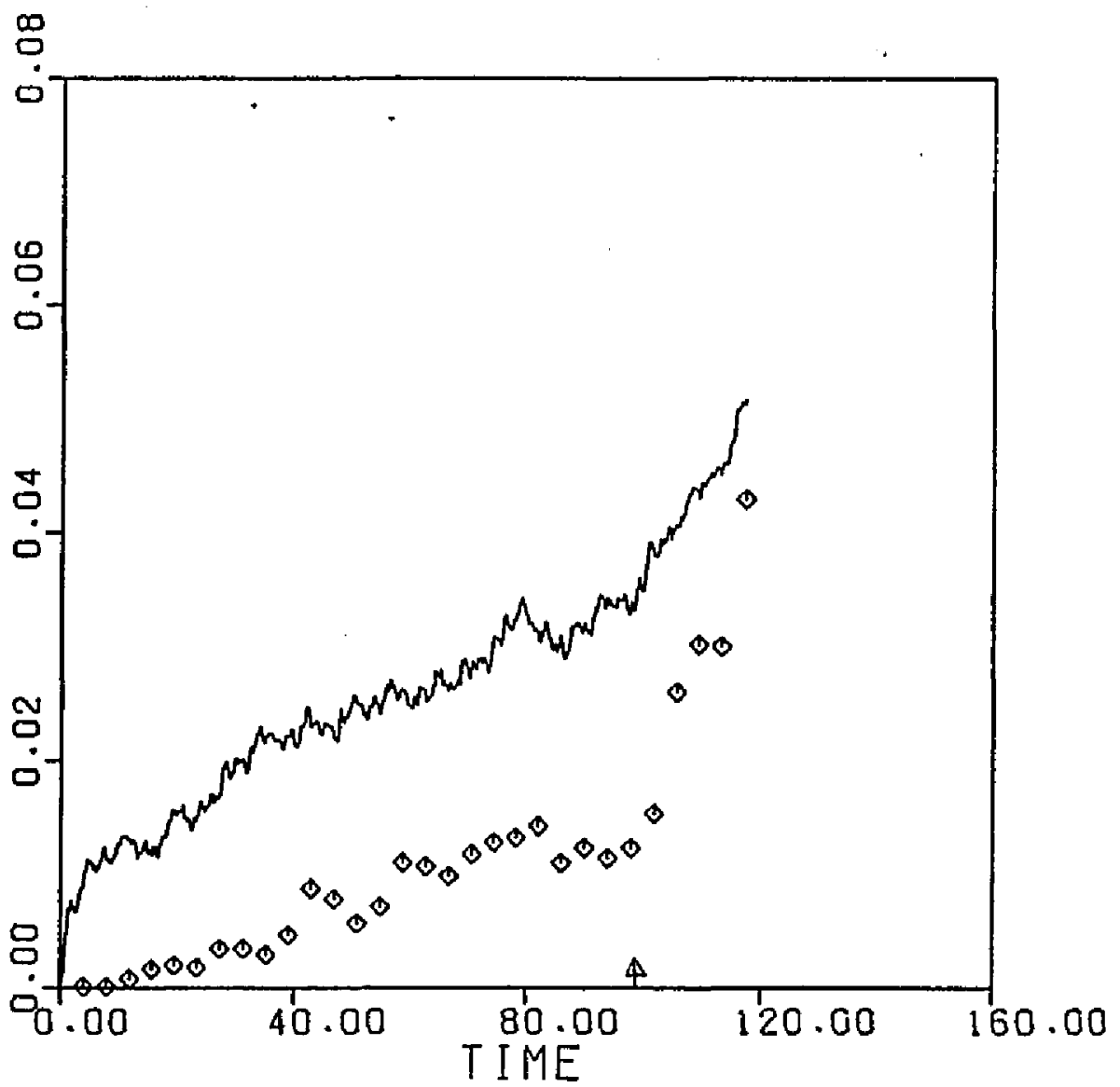


FIGURE $12 \mathrm{~A}$

VECTOR POTENTJAL PECTRUH

AVERAGED OVER 5O SNAP SHOTS ENDED AT $T=97.66$

DRIVEN HHD (STAR) RUN

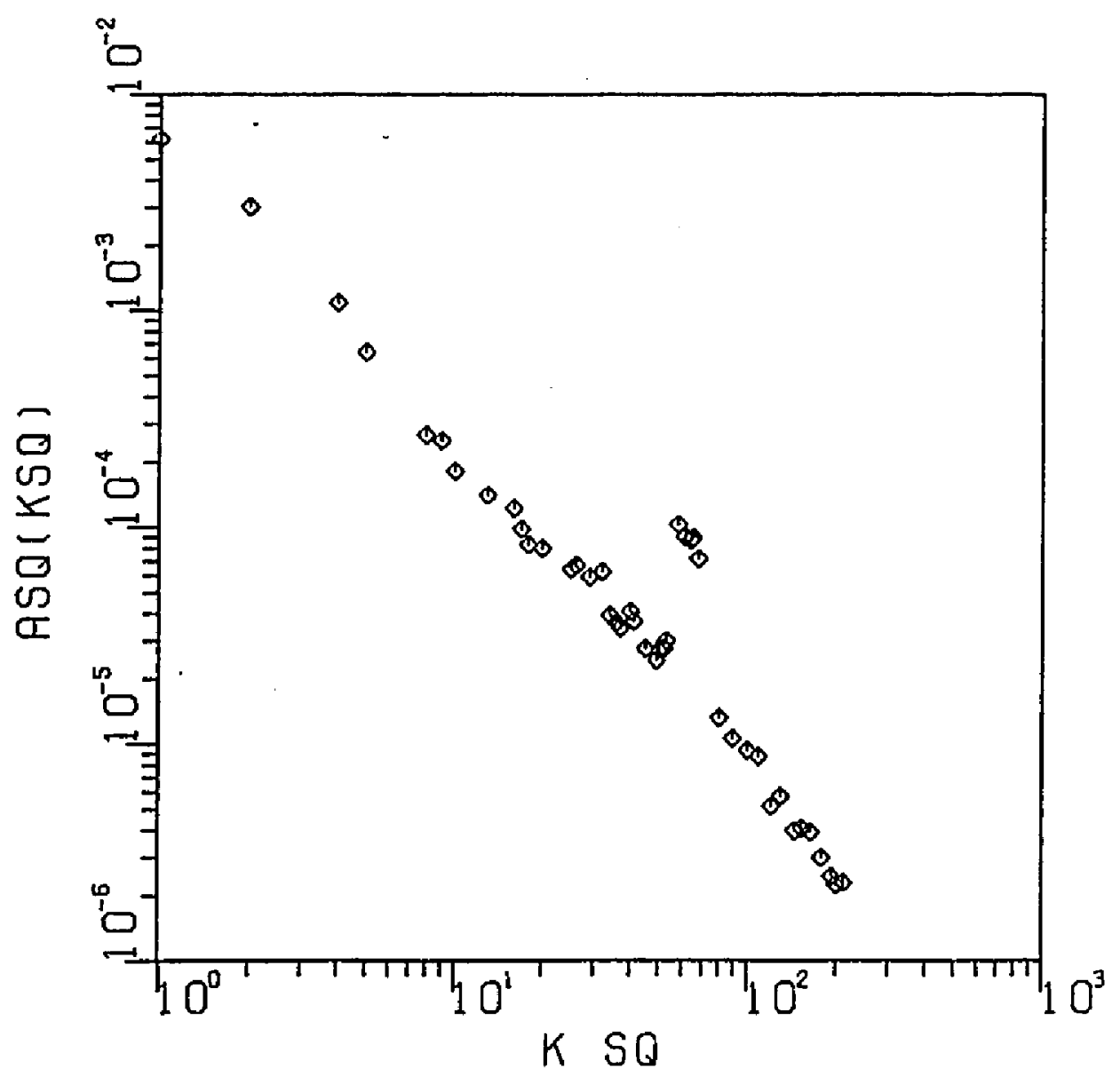


F I GURE 12B

MECHANICAL. MODAL ENERGY SPECTRUM

AVERAGED OVER SO SNAP SHOTS ENDED AT $T=97.66$

DRIVEN HHD (STAR) RUN

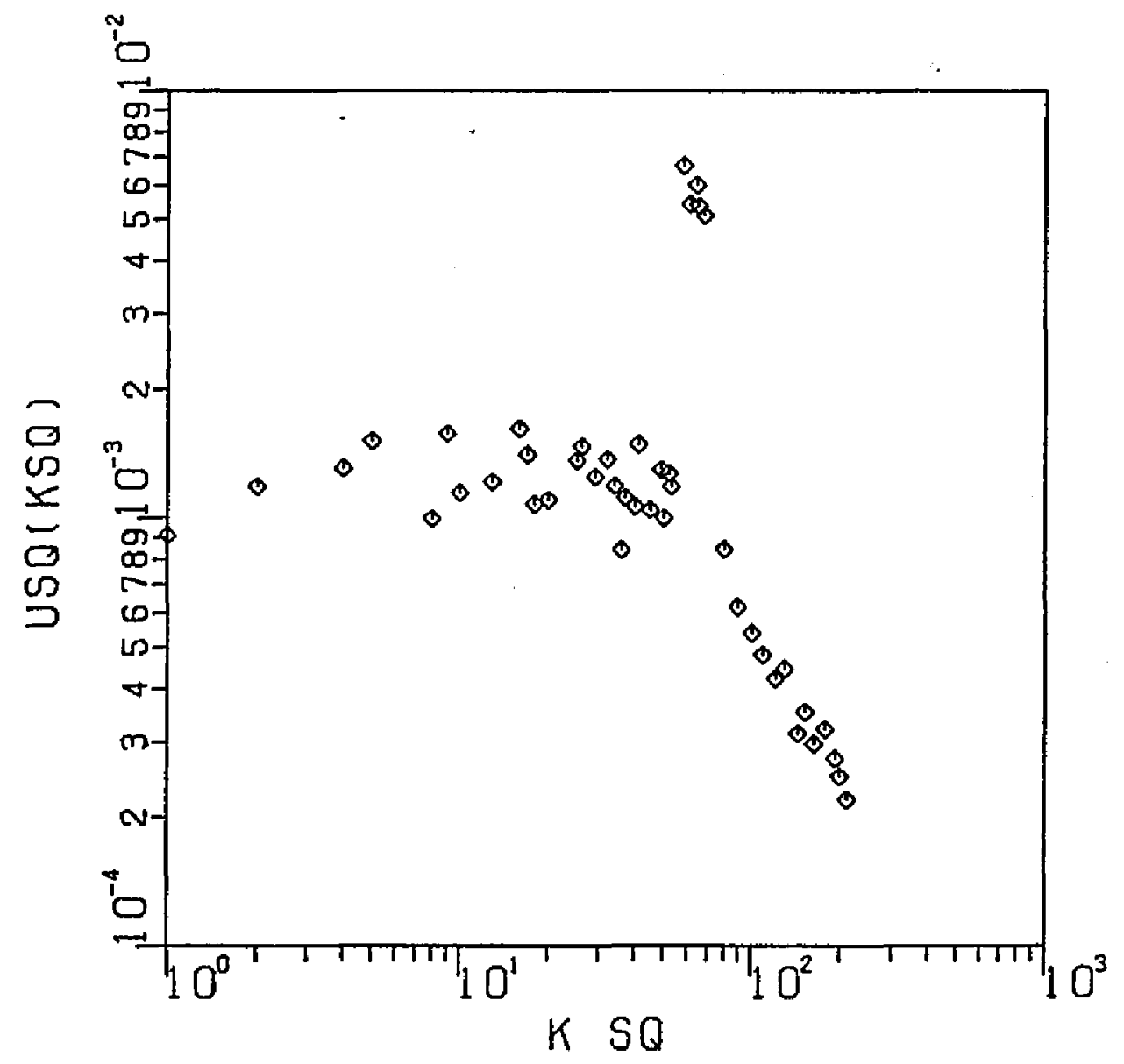




\section{FIGURE 12C}

MAGNETIC MODAL ENERGY SPECTRUH

AVERAGED OVER 5O SNAP SHOTS ENOEO RT $T=97.66$

DRIVEN MHO (STAR) RUN

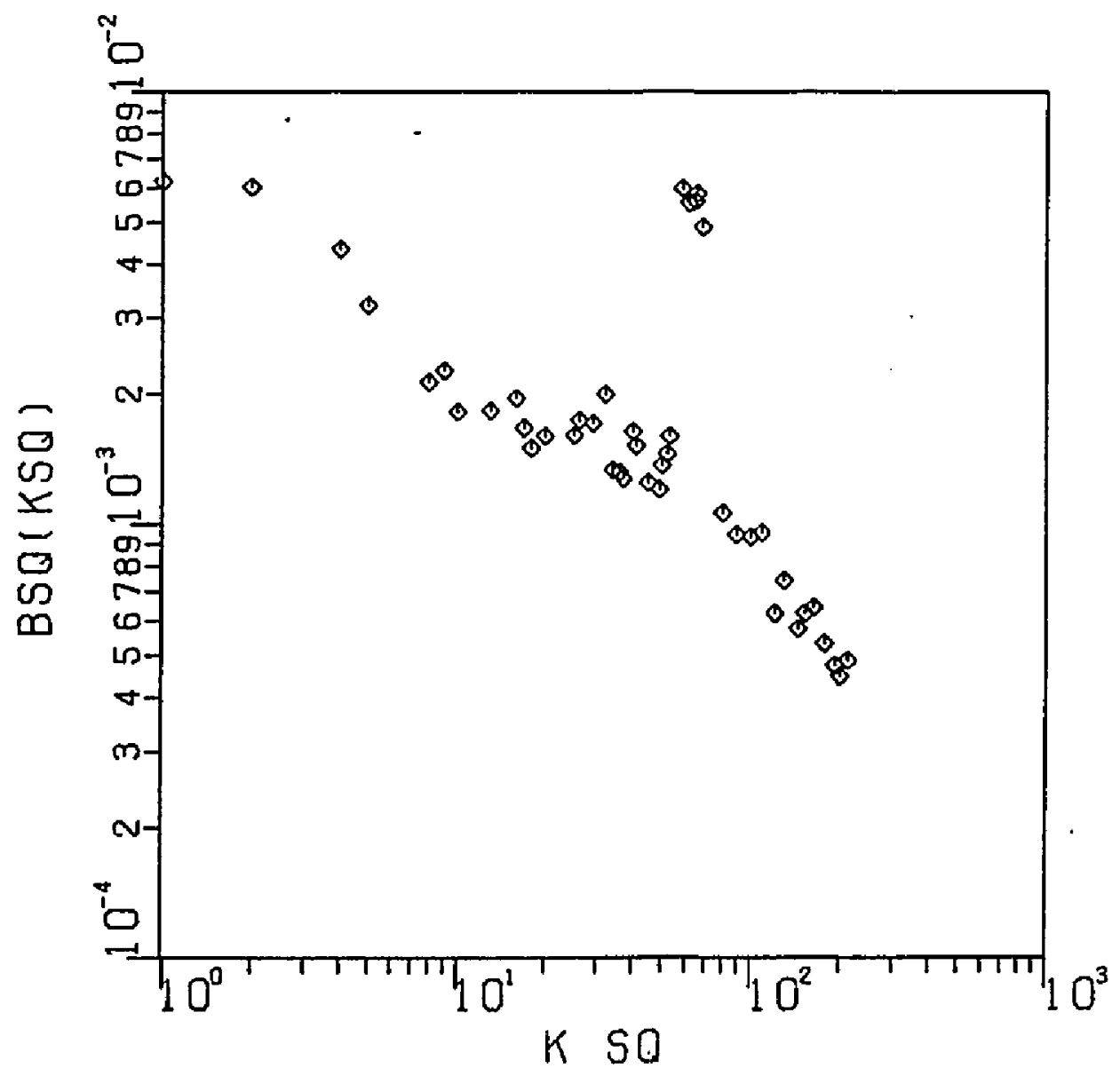




\section{FI.GURE 120}

VECTOR POTENTIAL PECTRUM

GYERAGED OVER 25 SMAP SHOTS ENOED AT $T=117.19$

DRIVEN MHD (STAR) RUN

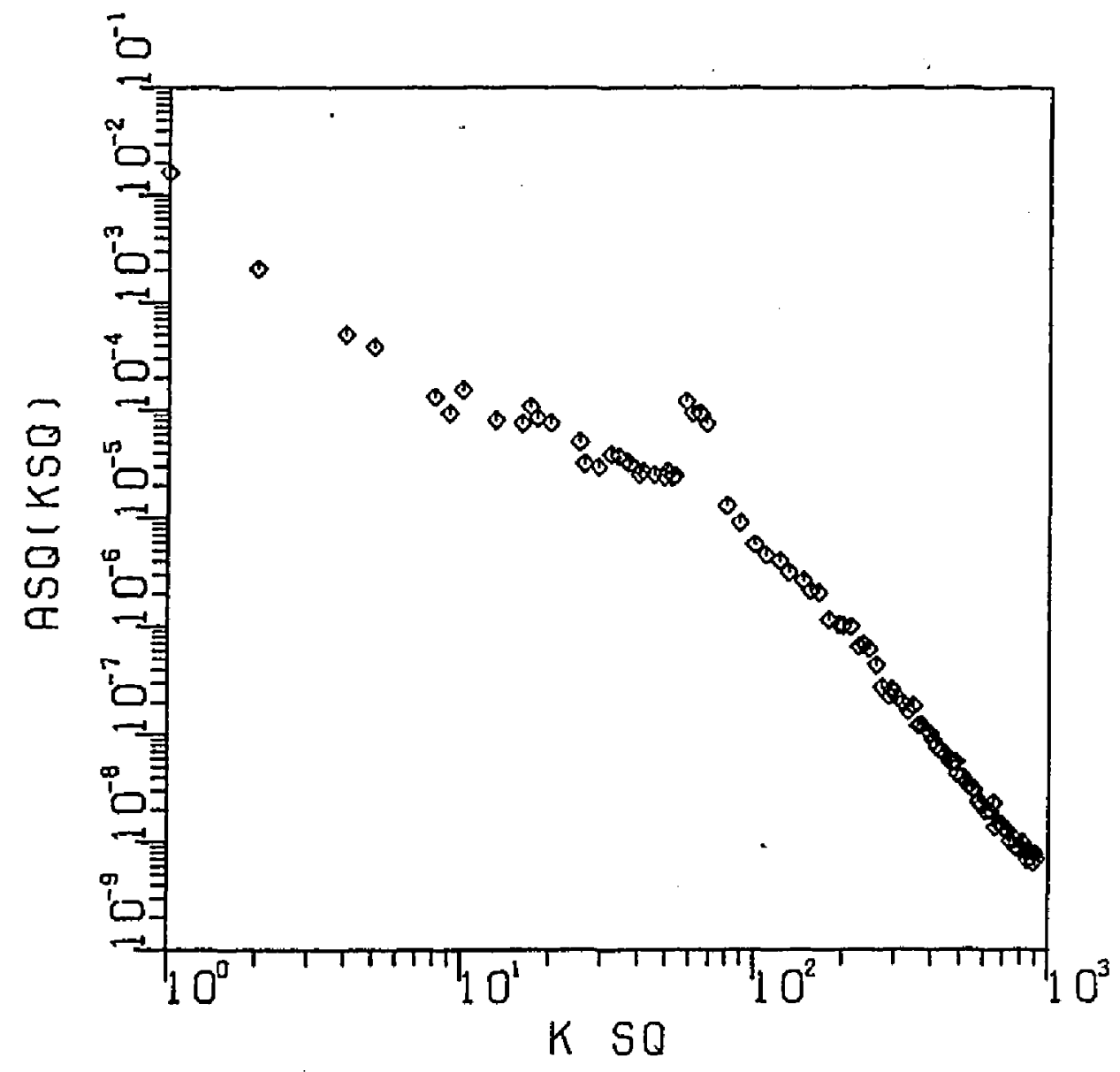


F I GURE 12E

HECHANICAL HODAL ENERGY SPECTRUH

AVERAGED OVER 25 SNAP SHOTS ENDED AT $T=117.19$

ORIVEN HHD (STAR) RUN

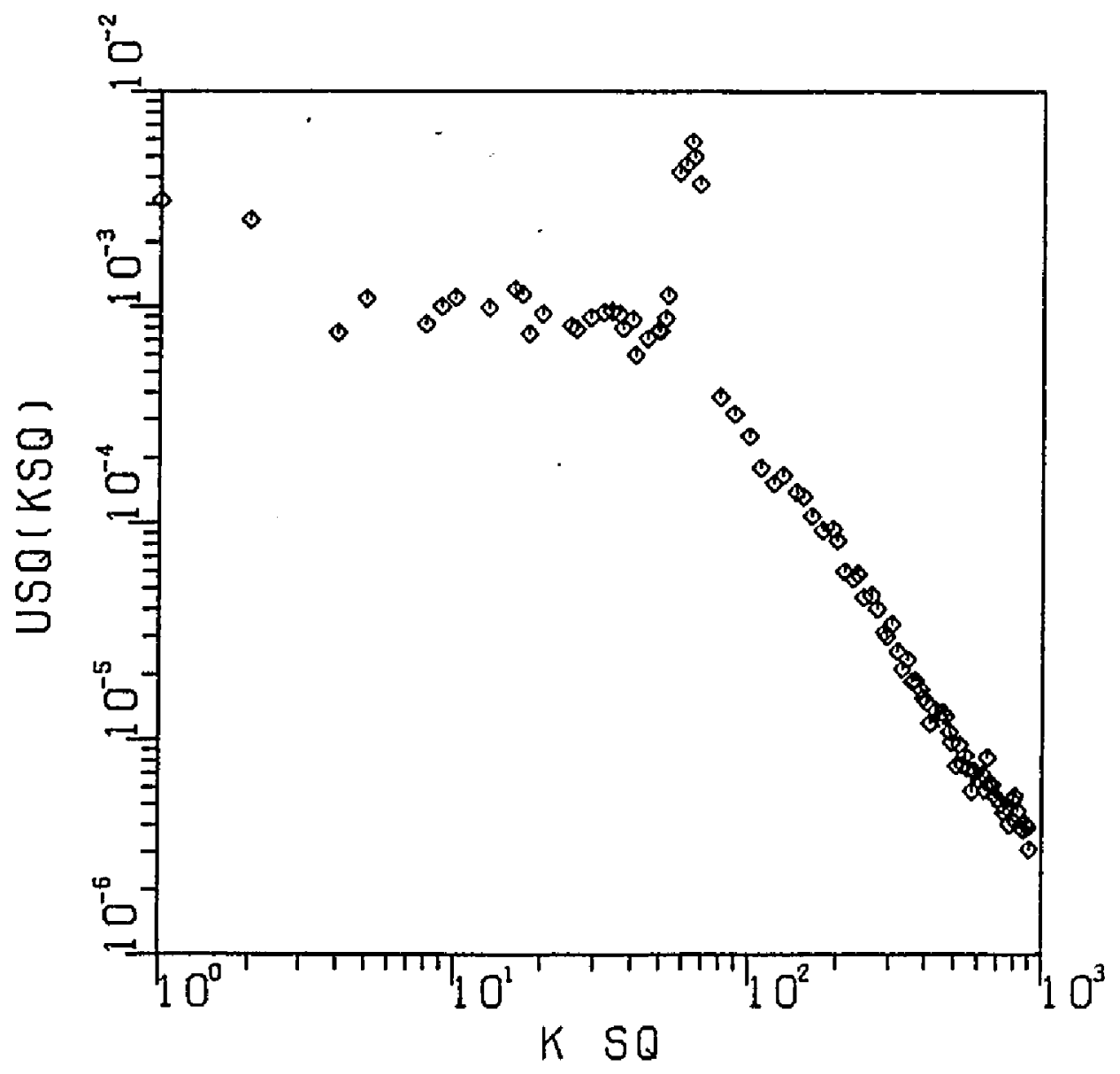


F I GURE $12 \mathrm{~F}$.

MAGNETIC MODAL ENERGY SPECTRUM

AVERAGED OVER 25 SNAP SHOTS ENDED AT $T=117.19$

DRIVEN MHO (STAR) RUN

SOLIO LINES ARE

POHER LAH LEAST SOUARE FIT

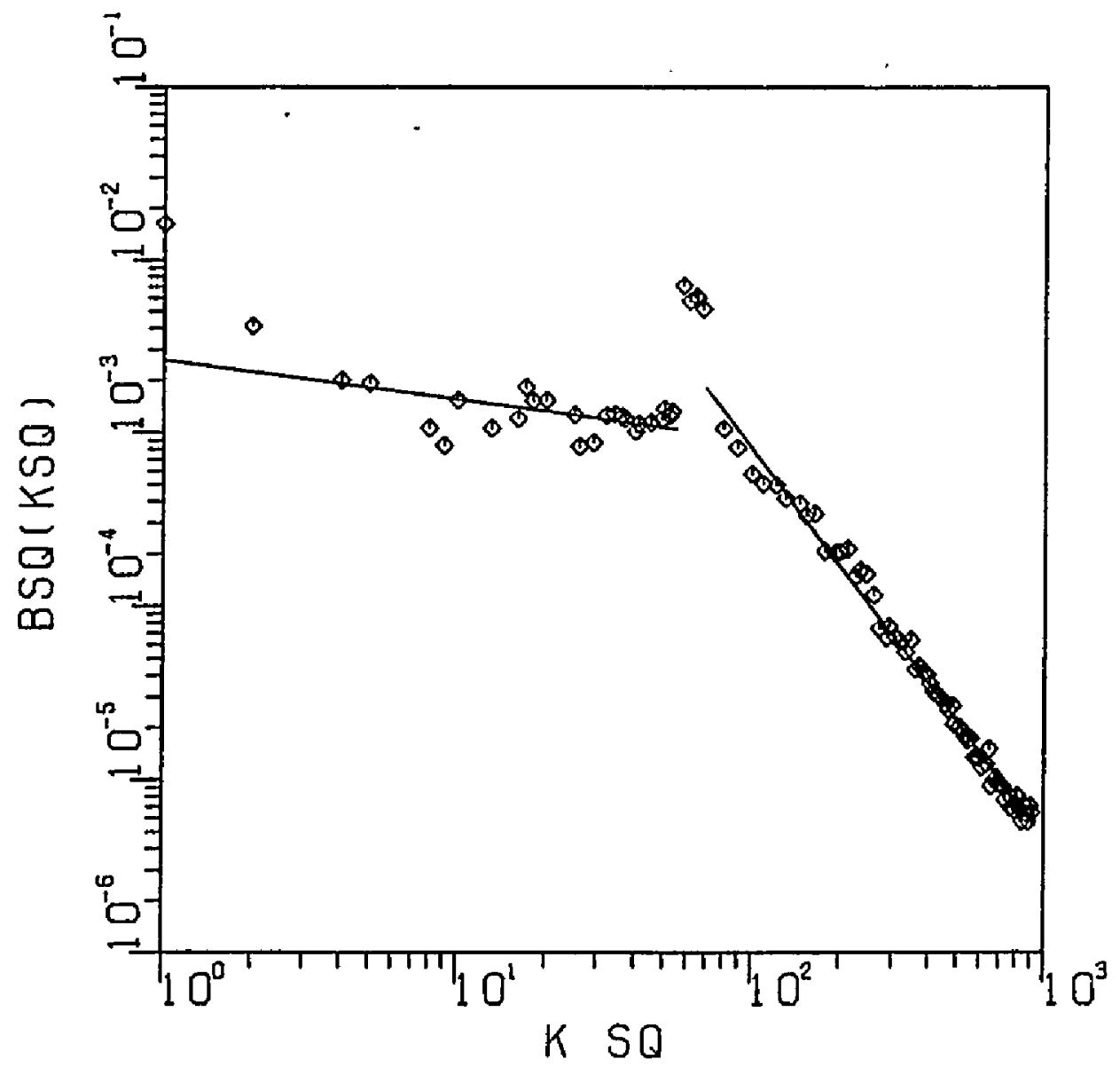


FIGURE 13A

CONTOURS OF VECTOR POTENTIAL AT $T=117.19$ TIME STEP 30000 DRIVEN HHD ISTAR \ RUN

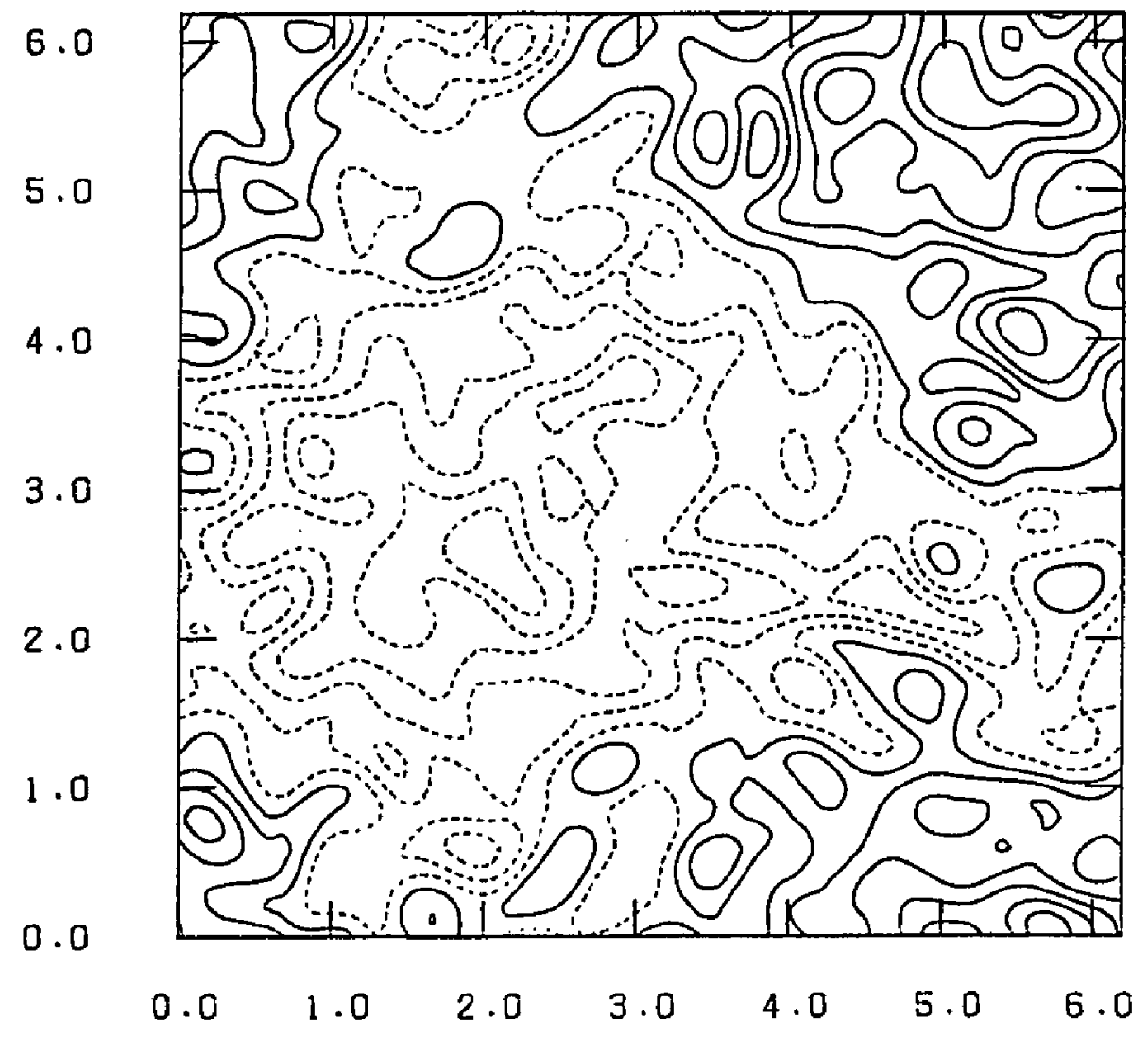




\title{
FIGURE 13B
}

\author{
CONTOURS OF VORTICITY DENSITY \\ AT $T=117.19$ TIHE STEP 30000 \\ DRIVEN MHD (STAR) RUN
}

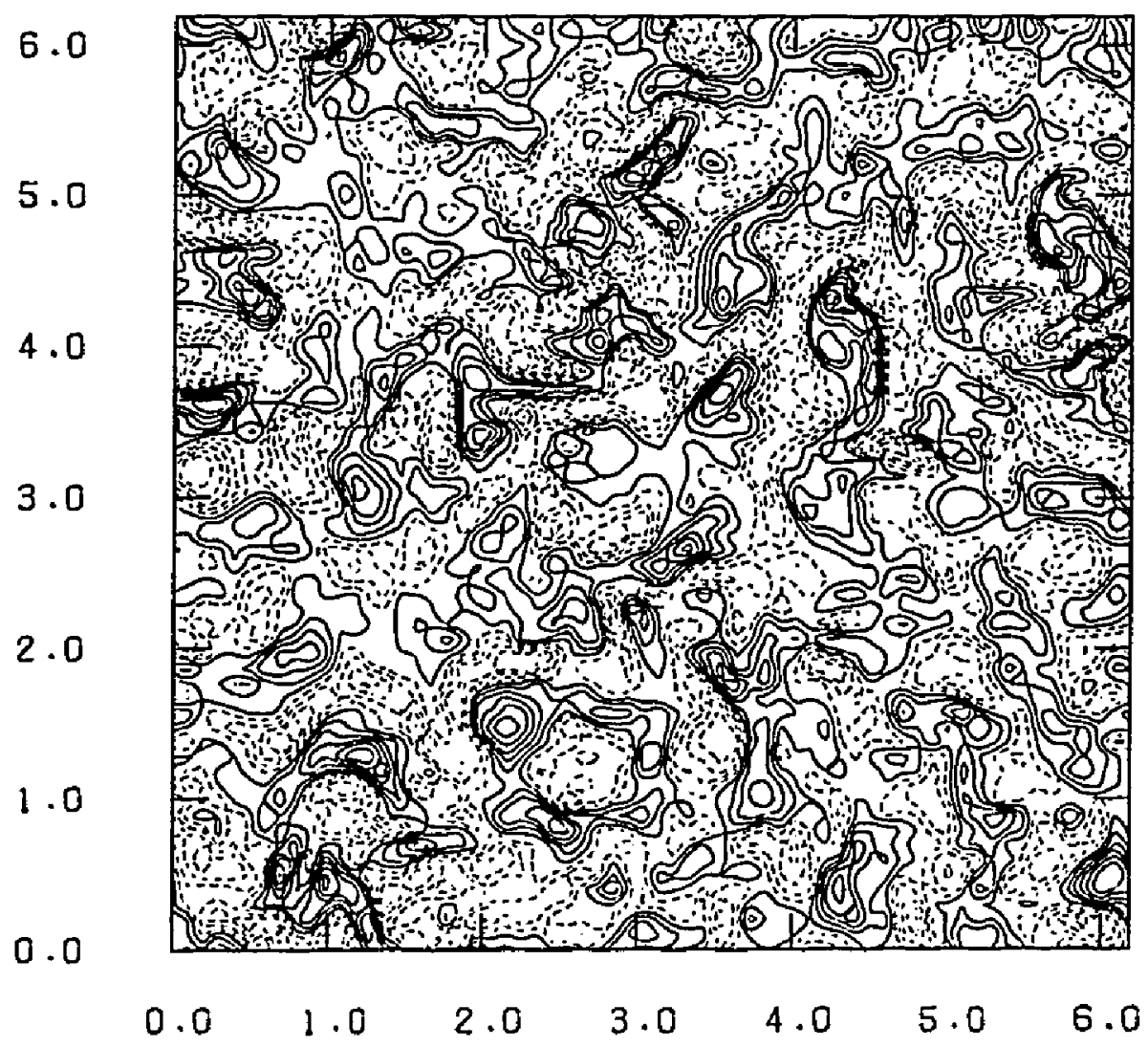




\title{
FIGURE $13 C$
}

\author{
CONTOURS OF CURRENT OENS! TY \\ RT $T=117.19$ TIHE STEP 30000 \\ DRIVEN MHO (STAR) RUN
}

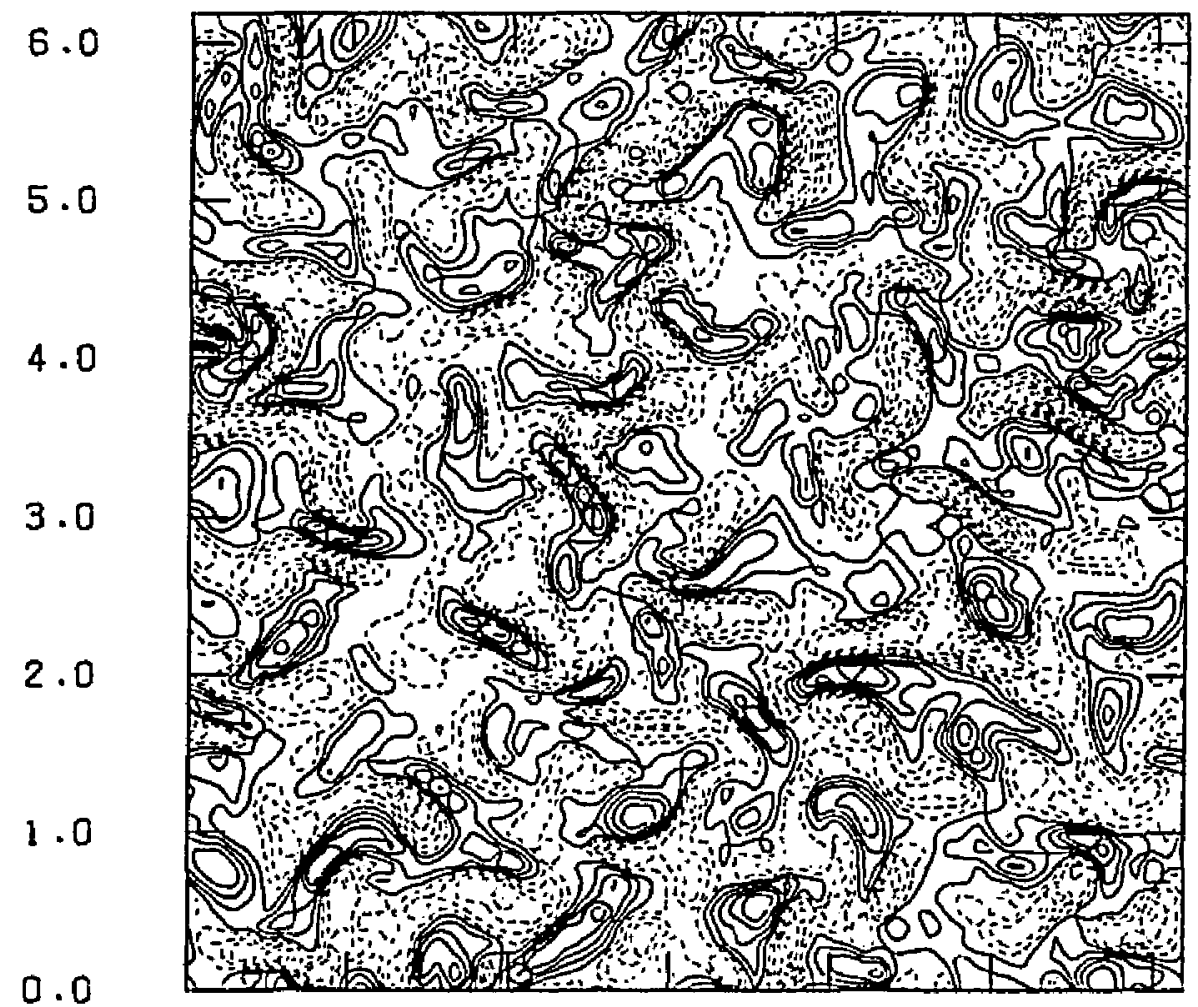
$0.0 \quad 1.0$
$2 \cdot 0$
$3 \cdot 0$
$4 \cdot 0$
$5 \cdot 0$
6.0 


\section{F I GURE 130}

CONTOURS OF STREAM FUNCTION

AT $T=117.19$ TIME STEP 30000

DRIVEN HHD (STAR) RUN

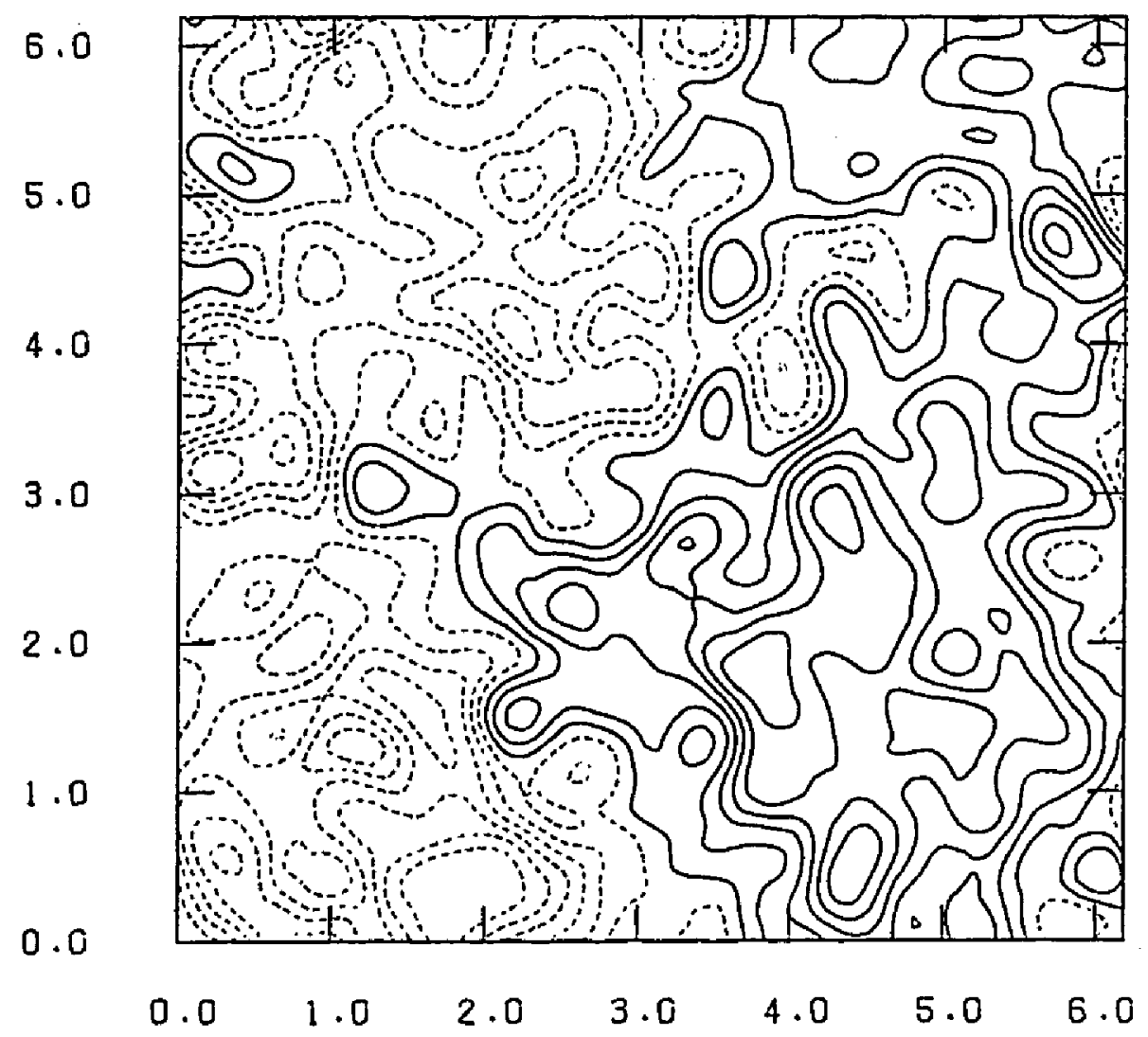


FIGURE 14A

ENERGY VERSUS TIME

PHASE RANDOMIZEO NAVIER-STOKES RUN

RRROH SHOHS HHEN PHASES HERE RANOOHIZED

CROSSES REPRESENT PHASE RANDOMIZED RUN

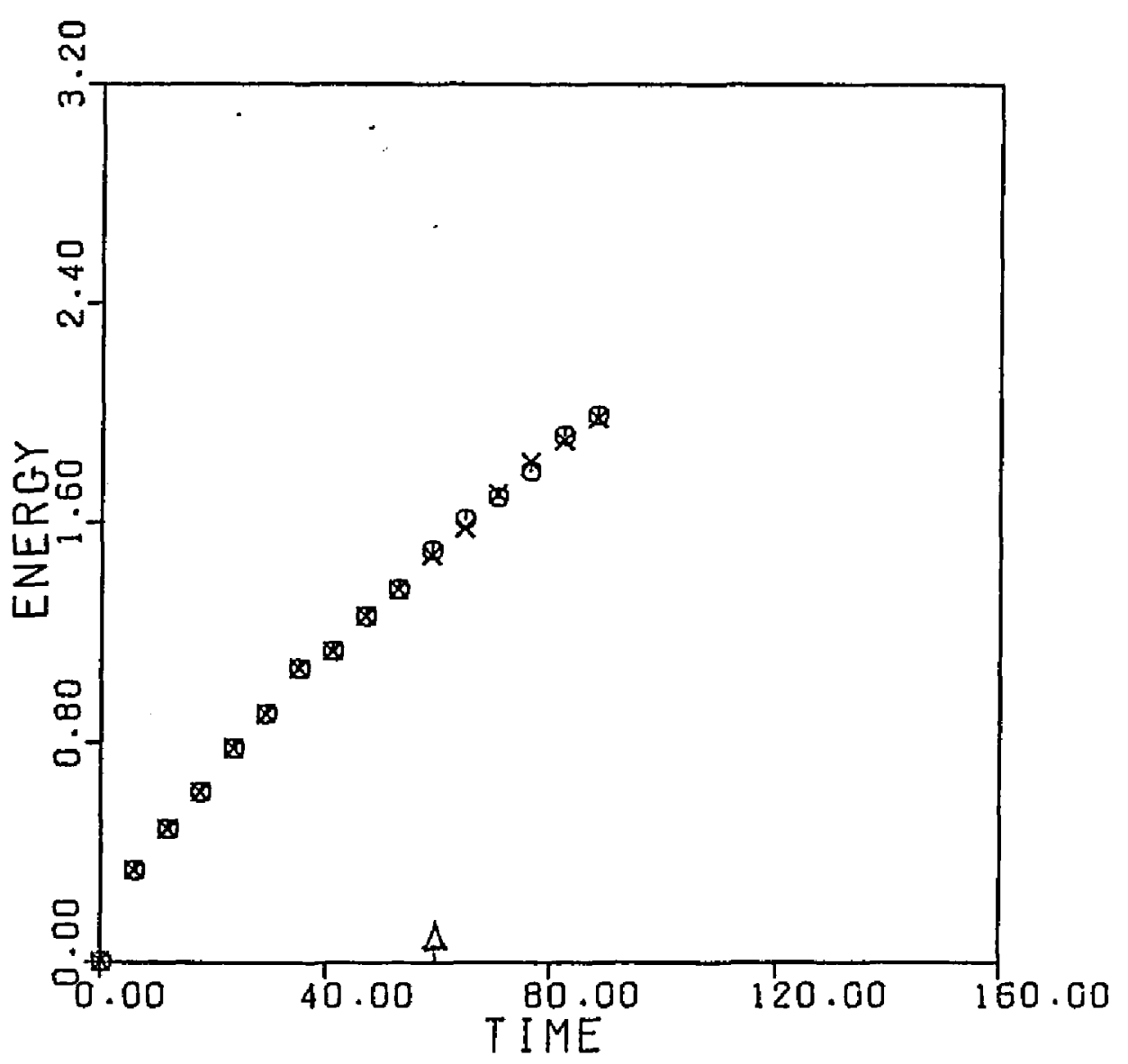


FIGURE 14B

MODRL ENERGY SPECTRA

PHASE RANDOMIZED NAYIER-STOKES RUN

AVERAGED OVER 1000 TIME STEPS ENDED AT $T=97.66$

CROSSES REPRESENT PHASE RANDOMIZED RUN

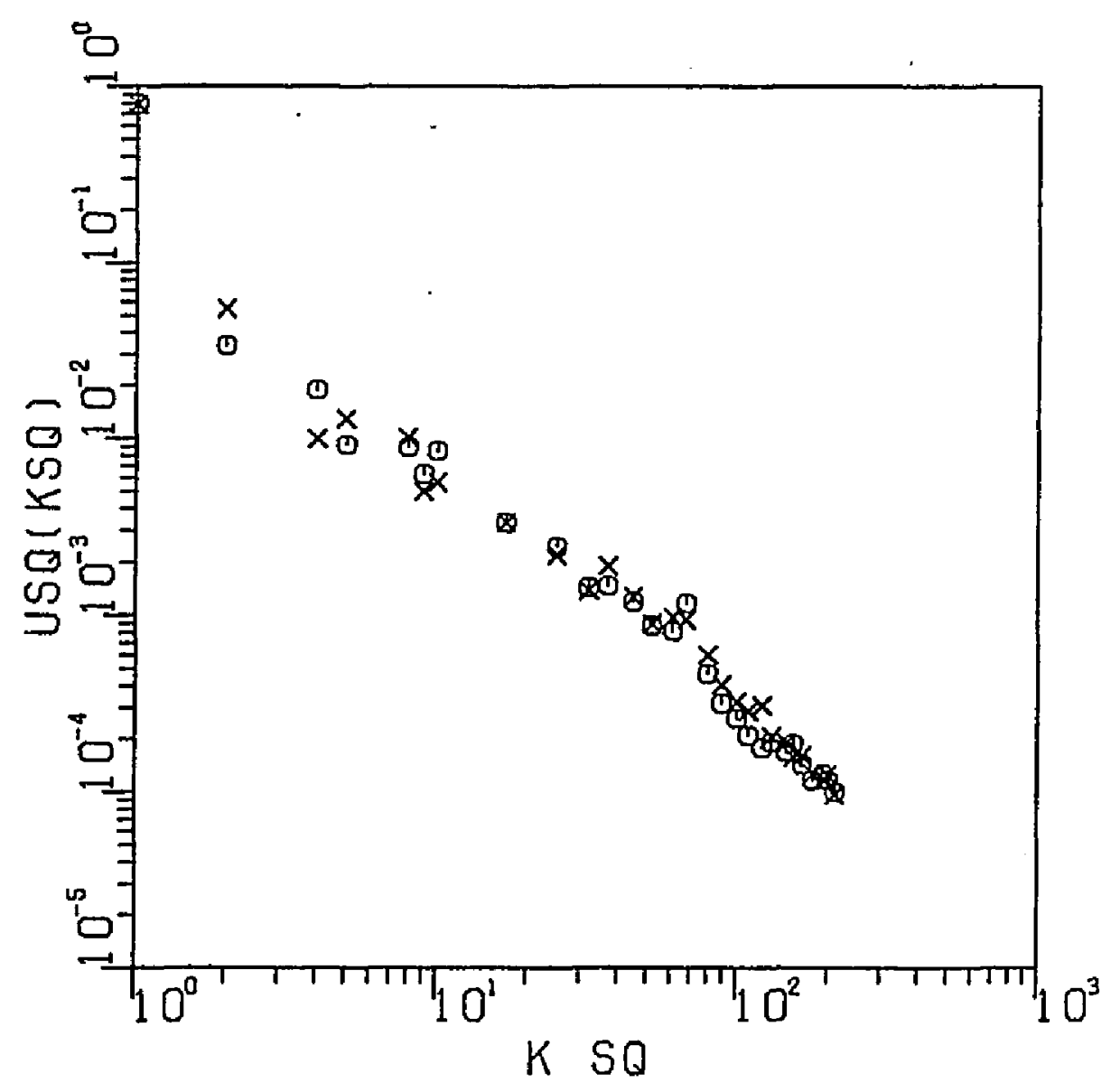




\section{FIGURE 15A}

HODEL FIT ON ENERGY VERSUS TIME CURVE FORCED NAVIER-STOKES RUN

DATA FROM LEAST SOUARE FIT LABELED AS D

DATA FROH MODEL CALCULATION LABELED AS M

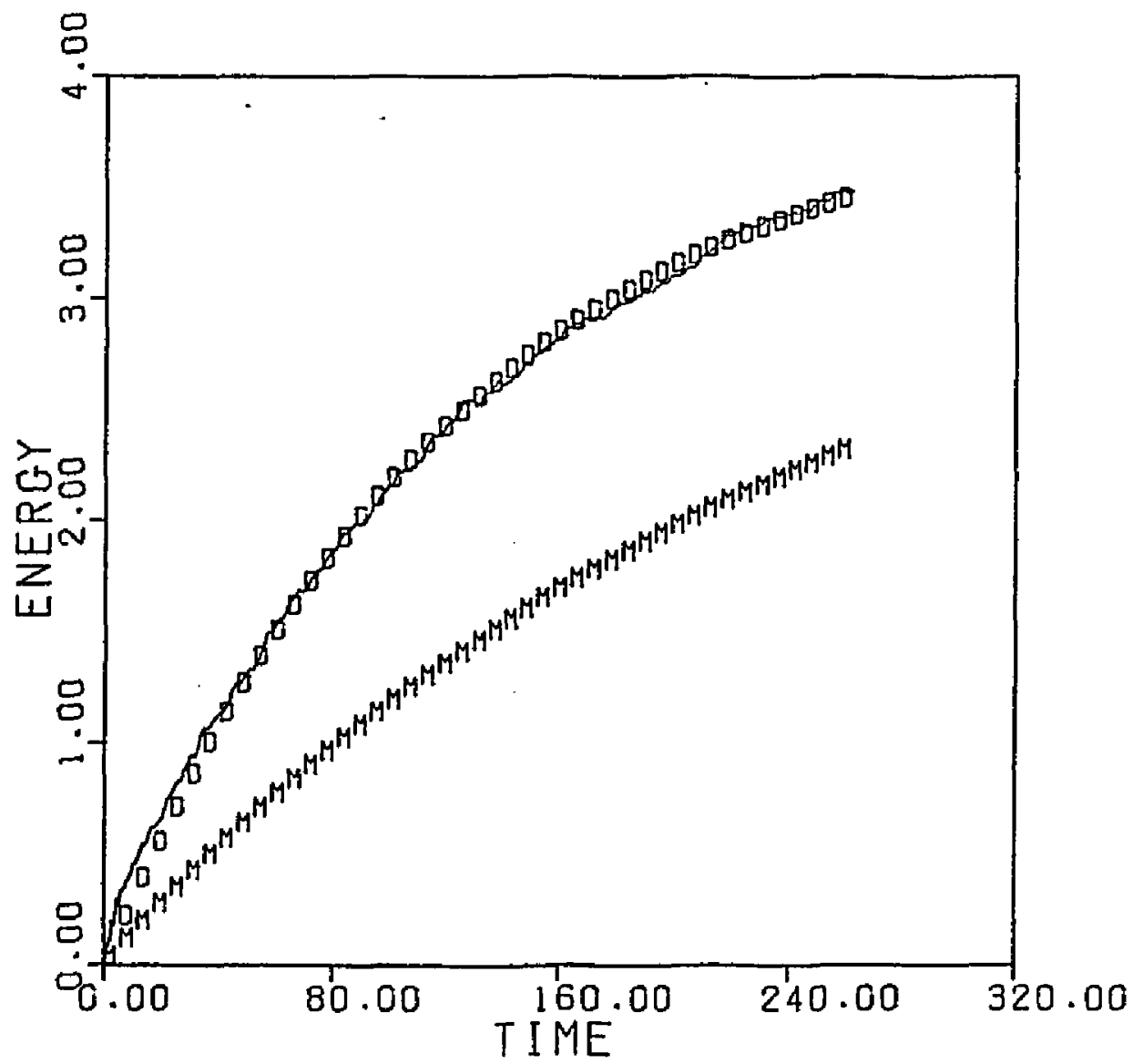


FIGURE 15B

MODEL FIT ON VECTOR POTENTIAL VS TIME CURVE FORCED HHD RUN

DATA FROM MODEL CALCULATION LABELED AS H

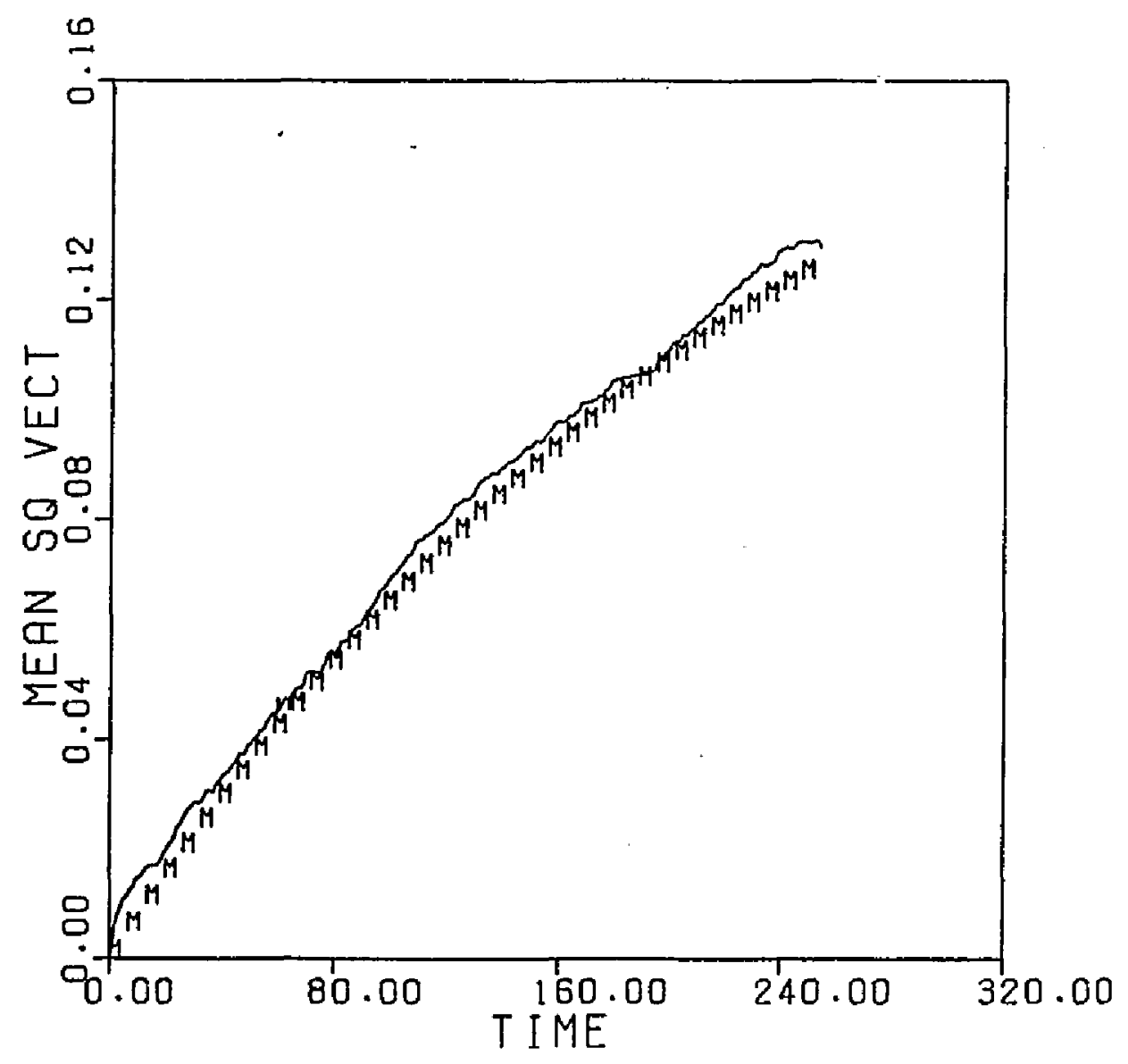


F IGURE 15C

TEST OF THE FINAL STATE FOR THE

FORCED NAVIER-STOKES RUN

ENERGY RT THE JUHP MADE HIGHER BY HANO

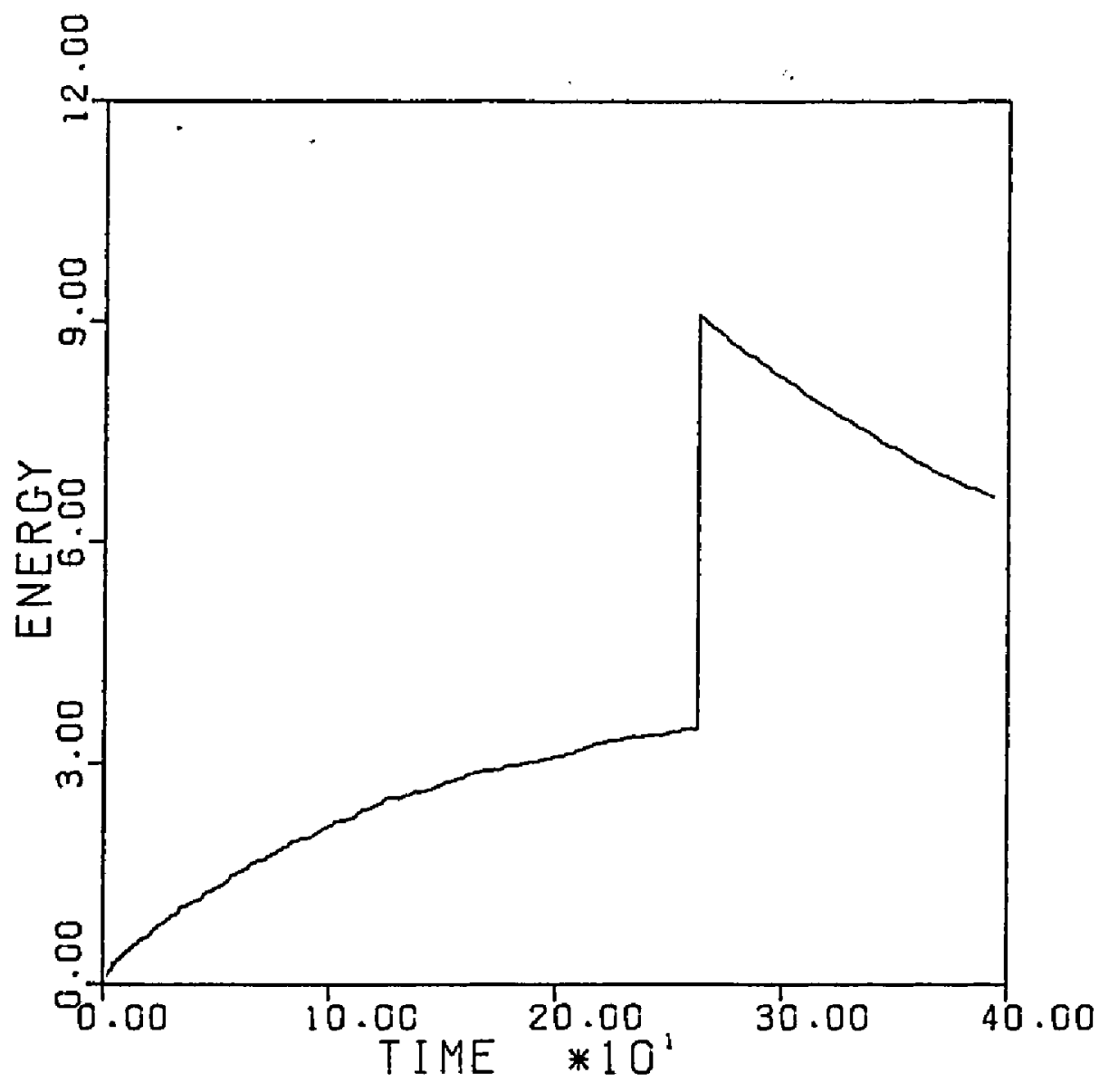


VITA

Murshed Hossain

Born in Phathaliakandi, Homn, Comilla,
Bangladesh, on November 21, 1950 . Passed the
Secondary School Certificate Examination from
Intermediate Technical College in 1967, Higher
Secondary Certificate Examination from Dacca College
in 1969. Graduated from the University of Dacca,
Bangladesh with B.Sc(Honours) and M.Sc. degrees in
Physics in 1975 and 1976 respectively.
Vorked for the Forest Research Institute,
Chittagong, Bangladesh and the Bangladesh Atomic
Energy Commission, Dacca before joining the College
of Villiam and Mary in 1979 . Received an M.S. in
1981 from the College.

